\title{
Multipoint observations of plasma phenomena made in space by Cluster
}

\author{
M. L. Goldstein ${ }^{1} \uparrow$, P. Escoubet ${ }^{2}$, K.-Joo Hwang ${ }^{1,3}$, D. E. Wendel ${ }^{1}$, \\ A.-F. Viñas ${ }^{1}$, S. F. Fung ${ }^{1}$, S. Perri ${ }^{4}$, S. Servidio ${ }^{4}$, J. S. Pickett ${ }^{5}$, G. K. Parks ${ }^{6}$, \\ F. Sahraoui ${ }^{7}$, C. Gurgiolo ${ }^{8}$, W. Matthaeus ${ }^{9}$ and J. M. Weygand ${ }^{10}$ \\ ${ }^{1}$ NASA Goddard Space Flight Center, Greenbelt, MD 20771, USA \\ ${ }^{2}$ ESA/ESTEC, Noordwijk, the Netherlands \\ ${ }^{3}$ University of Maryland at Baltimore County, Baltimore, MD 21250, USA \\ ${ }^{4}$ Dipartimento di Fisica, Università della Calabria, I-87036 Rende, Italy \\ ${ }^{5}$ Department of Physics and Astronomy, University of Iowa, Iowa City, IA 52242, USA \\ ${ }^{6}$ Space Sciences Laboratory, University of California, Berkeley, CA 94720, USA \\ ${ }^{7}$ Laboratoire de Physique des Plasmas, CNRS-Ecole Polytechnique-UPMC, Observatoire de \\ Saint-Maur, 94107 Saint-Maur-des-Fossés, France \\ ${ }^{8}$ Bitterroot Basic Research, Hamilton, MT 59840-9369, USA \\ ${ }^{9}$ Department of Physics and Astronomy, University of Delaware, Newark, DE 19716, USA \\ ${ }^{10}$ Institute of Geophysics and Planetary Physics, Department of Earth and Space Sciences, \\ University of California, Los Angeles, CA 90095, USA
}

(Received 21 August 2014; revised 3 February 2015; accepted 3 February 2015; first published online 11 March 2015)

Plasmas are ubiquitous in nature, surround our local geospace environment, and permeate the universe. Plasma phenomena in space give rise to energetic particles, the aurora, solar flares and coronal mass ejections, as well as many energetic phenomena in interstellar space. Although plasmas can be studied in laboratory settings, it is often difficult, if not impossible, to replicate the conditions (density, temperature, magnetic and electric fields, etc.) of space. Single-point space missions too numerous to list have described many properties of near-Earth and heliospheric plasmas as measured both in situ and remotely (see http://www.nasa.gov/missions/\#.U1mcVmeweRY for a list of NASA-related missions). However, a full description of our plasma environment requires three-dimensional spatial measurements. Cluster is the first, and until data begin flowing from the Magnetospheric Multiscale Mission (MMS), the only mission designed to describe the three-dimensional spatial structure of plasma phenomena in geospace. In this paper, we concentrate on some of the many plasma phenomena that have been studied using data from Cluster. To date, there have been more than 2000 refereed papers published using Cluster data but in this paper we will, of necessity, refer to only a small fraction of the published work. We have focused on a few basic plasma phenomena, but, for example, have not dealt with most of the vast body of work describing dynamical phenomena in Earth's magnetosphere, including the dynamics of current sheets in Earth's magnetotail and the morphology of the dayside high latitude cusp. Several review articles and special publications are available that describe aspects of that research in detail and interested readers are referred to them (see for example, Escoubet et al. 2005 Multiscale Coupling of Sun-Earth Processes, p. 459, Keith et al. 2005 Sur. Geophys. 26, 307-339, Paschmann et al. 2005 Outer Magnetospheric Boundaries: Cluster Results, Space Sciences Series of ISSI. Berlin: Springer, Goldstein et al. 2006 Adv. Space Res. 38, 21-36, Taylor et al.

$\dagger$ Email address for correspondence: melvyn.l.goldstein@nasa.gov 
2010 The Cluster Mission: Space Plasma in Three Dimensions, Springer, pp. 309-330 and Escoubet et al. 2013 Ann. Geophys. 31, 1045-1059).

\section{Cluster as a three-dimensional probe}

The four Cluster spacecrafts were launched a month apart into a polar orbit of $4 \times 19.6 \mathrm{R}_{E}$ by two Soyuz-Fregat rockets from Baikonur on 16 July and 9 August 2000 (Escoubet et al. 2001). The operational design and capability of being able to arrange the orbits so that the four spacecraft would form the vertices of a regular tetrahedron in areas of scientific interest meant that, for the first time one could make measurements in three dimensions with the ability to distinguish between spatial and temporal changes. The capability of being able to change the spacecraft separation, paired with the evolution of the orbit, opened new regions of the Earth's plasma environment to exploration. Cluster is very much an active mission - the separation of the spacecraft has been changed more than 25 times, as shown in Fig. 1. The separation distances have ranged from 4 to $36000 \mathrm{~km}$. In 2005, to study magnetofluid turbulence in the solar wind, the spacecraft separation was adjusted to form a 10000 $\mathrm{km}$ tetrahedron. Because spacecraft $3(\mathrm{C} 3)$ is in a very similar orbit to spacecraft 4 (C4), those two have, on occasion, been moved to within $4 \mathrm{~km}$ of each other. ESA has recently extended Cluster through 2016 with a possible further extension to 2018.

\subsection{General outline}

After a description of the instrumentation on the Cluster mission (Sec. 1.2) and a short section indicating where one can obtain Cluster data (Sec. 1.3), we describe in Sec. 1.4 how having four-point measurements permits one to explore various volumetric properties of the magnetofluid that comprise the magnetosphere and solar wind. We concentrate on a few investigations that emphasize fairly general plasma physics phenomena. Not covered are the many fundamental discoveries related to, for example, the generation of substorms. A general discussion of dynamical phenomena that Cluster has helped to elucidate can be found in Escoubet et al. (2013).

In Sec. 2, we discuss several plasma phenomena that have been studied using data from Cluster. Examples include magnetic reconnection (Sec. 2.1), where we describe some observations made within the ion diffusion region (IDR) and near, if not inside, the electron diffusion region (EDR). Included is a discussion of observations of magnetic nulls (Sec. 2.1.2), regions of particle acceleration (Sec. 2.2), especially due to the development and motion of dipolarization fronts (DFs) and betatron and stochastic mechanisms (Sec. 2.2.2). In Sec. 2.3, we discuss properties of various classes of plasma waves, including surface waves driven by velocity shears. Cluster data have also been used to investigate properties of high frequency plasma waves related to planetary radio emissions, including Auroral Kilometric Radiation (AKR) and Chorus, as well as various solitary waves (see Sec. 2.4.3). The spacecraft crossings of the Earth's bowshock have provided numerous opportunities to study the structure of collisionless shock waves and to test ideas of entropy conservation (Sec. 2.5).

Because we cannot review all of the phenomena investigated using Cluster data, we refer the interested reader to list of such studies (Taylor et al. 2010).

\subsection{Cluster instrumentation}

The Cluster payload is designed to study plasma phenomena and is arguably the most complete payload launched thus far. Some capabilities are unique and will 


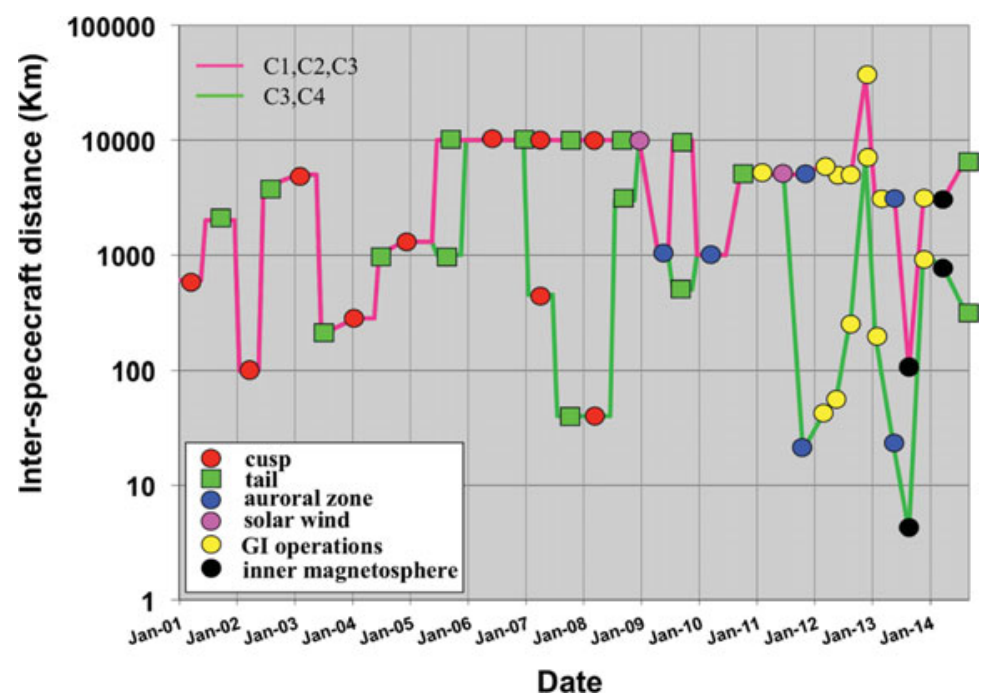

FIGURE 1. The inter-spacecraft distance over the course of the Cluster mission.

not be duplicated on MMS (although MMS will have much higher time resolution for plasma measurements). Following launch, 3 of the 44 instruments were not functioning (the Spacecraft POtential Control, ASPOC, on C1, the Composition Ion Spectrometer, CIS, on C2 and the Electron Drift Instrument, EDI, on C4). ASPOC, designed to control the spacecraft potential by emitting indium ions (Torkar et al. 2001), is now out of indium. During the $13 \mathrm{yr}$ of the mission, a few more instruments have experienced problems and have been switched-off and a few have reduced capability (for a general description of the mission, see Escoubet et al. 1997).

The 11 instruments, the current PI, and mass and power are shown in Table 1. The particle instruments generally have a time resolution of $4 \mathrm{~s}$, the spacecraft spin rate, but that can vary depending on the mode of the instrument. The various field instruments have a range of time resolutions. Details of the instruments, their modes, and time resolutions can be found in the special issue of Annales Geophysicae (see the introduction by Alcaydé 2001, and subsequent articles).

Fundamental to the success of any space plasma mission is to have accurate and reliable magnetometers. Cluster has two, covering different ranges of magnetic field intensity and time resolution. The flux gate magnetometer (FGM) measures from DC to a maximum of $67 \mathrm{~Hz}$ (Balogh et al. 2001) and the search coil magnetometer (STAFF) operates up to $4 \mathrm{kHz}$ (Cornilleau-Wehrlin et al. 2003) - both are operating on all four spacecraft. Cluster includes a sounder (WHISPER) (Décréau et al. 2001) that by exciting Langmuir or Upper Hybrid waves provides an accurate measurement of the local plasma density. The thermal electron plasma is measured by PEACE (Johnstone et al. 1997). Both WHISPER and PEACE are working on all four spacecraft. The Wide Band Data instrument, WBD, makes high-resolution measurements of electric and magnetic fields in frequency bands from $25 \mathrm{~Hz}$ to $577 \mathrm{kHz}$ (Gurnett et al. 2001). WBD and the Electric Fields and Waves instrument (EFW) (Gustafsson et al. 2001) are also operating on all four spacecraft. EFW measures quasi-static electric fields of amplitudes up to $700 \mathrm{mV} \mathrm{m}^{-1}$, waveforms with a bandwidth of $4 \mathrm{kHz}$, as well as providing estimates of the spacecraft potential. The thermal ion plasma instrument (CIS2/HIA) and ion composition instrument (CIS1/CODIF) (Reme et al. 2001) are currently working on two spacecraft (C1 
Instrument

ASPOC (Spacecraft potential control)

CIS $0<E<40 \mathrm{keV})$

(Composition ion spectrometer)

EDI (Electron drift instrument)

FGM (Fluxgate magnetometer)

PEACE $0<E<30 \mathrm{keV}$

(Plasma electron and current experiment)

RAPID (High energy electrons and ions)

$\mathrm{DWP}^{a}$ (Wave processor)

$\mathrm{EFW}^{a}$ (Electric field and waves)

STAFF $^{a}$ (Magnetic and electric fluctuations)

$\mathrm{WBD}^{a}$ (Electric field and wave forms)

WHISPER $^{a}$ (Electron density and waves)

Total

${ }^{a}$ Members of the wave experiment consortium (WEC)

${ }^{b}$ including power supply
Mass(kg) Power(W)

$\begin{array}{lrr}\text { K. Torkar (IRF, A) } & 1.9 & 2.7 \\ \text { I. Dandouras } & 10.8 & 10.6 \\ \text { (IRAP/CNRS, F) } & & \\ \text { R. Torbert (UNH, USA) } & 10.5 & 9.1 \\ \text { C. Carr (IC, UK) } & 2.6 & 2.2 \\ \text { A. Fazakerley (MSSL, UK) } & 6.0 & 4.2 \\ & & \\ \text { P. Daly (MPAe, D) } & 5.7 & 4.5 \\ \text { M. Balikhin (Sheffield, UK) } & 2.9^{b} & 4^{b} \\ \text { M. André (IRFU, S) } & 16.2 & 3.7 \\ \text { P. Canu (LPP, F) } & 5.0 & 2.8 \\ \text { J. Pickett (IOWA, USA) } & 1.8 & 1.7 \\ \text { J.-L. Rauch (LPC2E, F) } & 1.8 & 1.8 \\ & 65.2 & 47.3\end{array}$

TABLE 1. The 11 instruments on each of the four Cluster spacecraft.

for HIA and C4 for CODIF) and were working on three spacecraft (C1, C3, and C4) between 2001 and 2009. The energetic ion experiment (Research with Adaptive Particle Imaging Detectors- RAPID) is currently working on C2 and C4, while the energetic electron instrument (Imaging Electron Spectrometer, IES) is operating on all four spacecraft (Wilken et al. 2001). EDI uses an electron gun to provide the third component of the electric field (EFW provides the other two) when possible. EDI is operating on C1, C2, and C3 (Paschmann et al. 2001).

\subsection{Data availability}

The data products from Cluster are publicly available via the Cluster Science Archive (CSA). For a general description of the original Cluster Active Archive (CAA), see Laakso et al. (2010). The contents and configuration of the CAA and CSA are similar. The CSA includes all high resolution data, moments, etc., as well as graphical products, an inventory of data availability, and has several enhancements to the original CAA. Information about the various instrument modes, time resolution, etc., can also be found there. These data are available in both Cluster Exchange Format, an ASCII representation that includes relevant metadata, as well as the common data format (CDF).

\subsection{Spatial derivatives}

Critical to the three-dimensional analysis of these data is the ability to make volumetric calculations utilizing their tetrahedral configuration, which provides the opportunity to take first-order derivatives of plasma and field quantities (see, Paschmann and Daly 1998).

This capability has been exploited most notably in computing the curl of the magnetic field to provide an estimate of the total plasma current (Dunlop et al. 2002; Dunlop and Eastwood 2008). When applicable, the technique avoids the limitations of particle instruments where one is never certain that all of the lowest energy particles have been counted. The technique also avoids errors that can arise from the motion of current sheets, which is especially a problem in the magnetosphere where plasma structures move faster than the spacecraft. This general 'curlometer' technique has 
also been used to study, for example, mirror mode structures (Lucek et al. 2001). Detailed discussions of the procedure as applied to currents and vorticity can be found in Chanteur (1998) and Gurgiolo et al. (2010, 2011).

Spatial derivatives of a scalar quantity $Q$, which may be the component of a vector, can be estimated as long as it is known at a minimum of four non-coplanar locations. With four data points, the variation of $Q$ across the volume defined by the spacecraft is provided by the set of linear equations:

$$
Q_{j}=a+b X_{j}+c Y_{j}+d Z_{j},
$$

where $Q_{j}$ is the quantity being fit, $j$ is the spacecraft index, $(a, b, c, d)$ are a set of unknown coefficients, and $(X, Y, Z)$ are the spacecraft position coordinates. For any $Q$, (1.1) represents four equations, one per spacecraft. If $Q_{j}$ represents a vector, then (1.1) must be solved for each component. By way of an explicit example, the equations for the quantity $Q_{x}$ are given by the coupled set of equations:

$$
\begin{aligned}
& C 1: Q_{x 1}=a_{x}+b_{x} X_{1}+c_{x} Y_{1}+d_{x} Z_{1} \\
& C 2: Q_{x 2}=a_{x}+b_{x} X_{2}+c_{x} Y_{2}+d_{x} Z_{2} \\
& C 3: Q_{x 3}=a_{x}+b_{x} X_{3}+c_{x} Y_{3}+d_{x} Z_{3} \\
& C 4: Q_{x 4}=a_{x}+b_{x} X_{4}+c_{x} Y_{4}+d_{x} Z_{4} .
\end{aligned}
$$

Estimates of both the divergence $(\nabla \cdot \mathbf{V})$ and $\operatorname{curl}(\nabla \times \mathbf{V})$ of any vector quantity $\mathbf{V}$ can be constructed from (1.2) with appropriate generalization for the vector components:

$$
\begin{gathered}
\nabla \cdot \mathbf{V}=b_{x}+c_{y}+d_{z}, \\
\nabla \times \mathbf{V}=\left(c_{z}-d_{y}\right) \widehat{x}+\left(d_{x}-b_{z}\right) \widehat{y}+\left(b_{y}-c_{x}\right) \widehat{z} .
\end{gathered}
$$

Using linear solutions to describe the spatial variations of measurements within the constellation volume has implications that need to be kept in mind. One major consequence is that the results depend explicitly on spacecraft separation. Consequently, use of (1.2) is restricted to time periods when the spacecraft are in a nearly regular tetrahedral configuration. The quality of the configuration can be judged from the tetrahedron geometry parameter (QGM) (Robert et al. 1998), which varies between 1 and 3 ( 3 indicates that the spacecraft are in a nearly regular tetrahedral geometry and 1 indicates the spacecraft are aligned as a string of pearls). The effect of magnitude skewing can be seen in Fig. 2, taken from Gurgiolo et al. (2013). They used (1.4) to compute the curl using an artificial set of magnetic field data (all components on all spacecraft set to $5000 \mathrm{nT}$ ) with actual spacecraft configuration data for an $11 \mathrm{~h}$ time period. The upper plot contains the estimates of the curl which should be 0 and the lower plot the QGM parameter. Jitter in the determined spacecraft positions is responsible for the noise in the curl. At about 15:30 UT (QGM $\approx 2.7$ ) the curl shows an upward trend as changes in the $(x, y, z)$ spacecraft separations move the spacecraft away from a regular tetrahedral configuration. A QGM of 2.7 should them be considered as the lower limit for applying (1.1) successfully. When using (1.1) one must also ensure that the measurements are independent, which is true so long as the average convection time of local features across the constellation occurs more slowly than the temporal resolution of the measurements. Consequently, measurements with longer cadences require larger spacecraft separation than do measurements made at shorter cadences. 

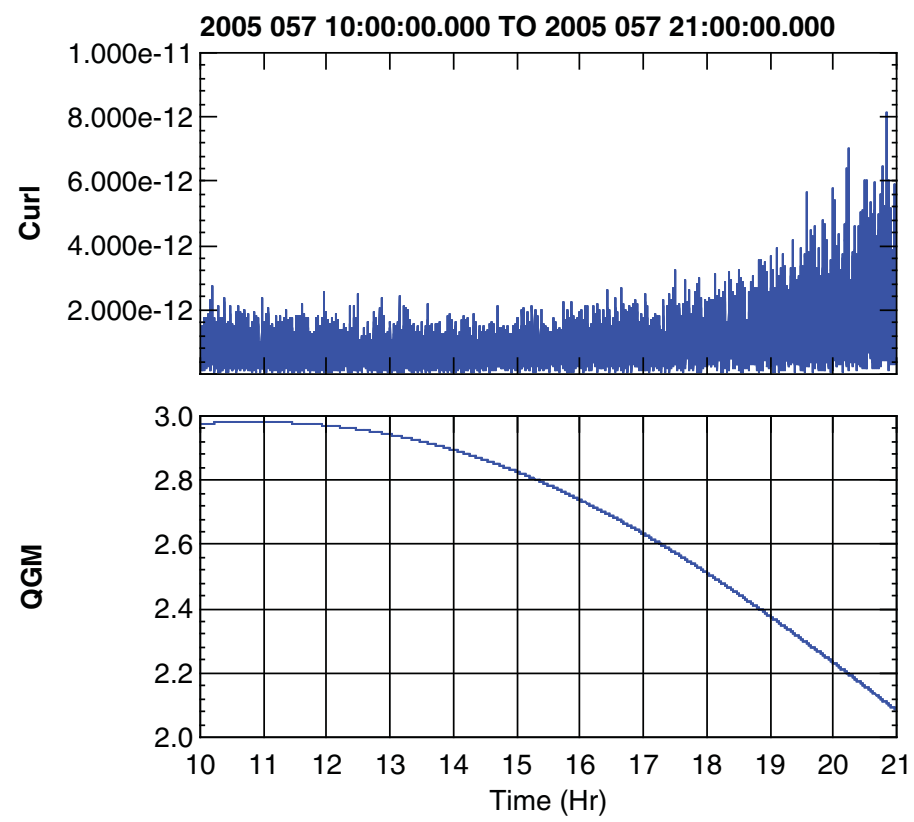

FIGURE 2. From Gurgiolo et al. (2013) showing the sensitivity of estimates of $\nabla \times \mathbf{B}$ on the configuration of Cluster over an $11 \mathrm{~h}$ period.

\section{Plasma phenomena as measured in three dimensions}

\subsection{Magnetic reconnection}

Magnetic reconnection converts magnetic energy into particle energy- often rapidly and efficiently (for a review, see Paschmann 2008). Understanding the physics behind this phenomenon is a continuing focus of study in both space and laboratory plasmas. The initiation and reconfiguration of magnetic topology associated with reconnection are thought to arise as a result of demagnetization of electrons within an EDR that is embedded within a much larger IDR where ions are demagnetized. Although IDRs, where Hall physics governs the magnetofluid description, have been identified by their magnetic and electric field geometry (see e.g., Wygant et al. 2005; Vaivads et al. 2006; Eastwood et al. 2010; Hwang et al. 2013), the EDR is difficult to observe because its size is of the order of a few electron inertial lengths, $d_{e}$, where $d_{e}=c / \omega_{p e}$ and $\omega_{\mathrm{pe}}$ is the electron plasma frequency. Macroscopic properties of reconnection are, however, evident in reconnection jets, i.e., accelerated plasmas outside of the small diffusion region, as well as by the reorganized magnetic topology. Particle moments and distributions in inflow and outflow regions have been used to estimate the reconnection rate at which magnetic energy is converted into particle energy (e.g., Fuselier et al. 2010; Lee et al. 2013).

Cluster data have elucidated aspects of reconnection that occurs in the solar wind, magnetosheath, and magnetosphere. Figure 3, based on Fig. 1 from Hwang et al. 2013, shows an example from the magnetosphere that has been chosen to emphasize the central importance of DFs. The event is from August 18, 2002. Plotted are magnetic (a), (d) and electric field (b), (e) and thermal ion (c), (f) and electron (g)-(j) moments obtained from $\mathrm{C} 4(\mathrm{a})-(\mathrm{c}),(\mathrm{j})$ and $\mathrm{C} 1$ (d)-(i). The initial background magnetic field 
A

1st flow reversal

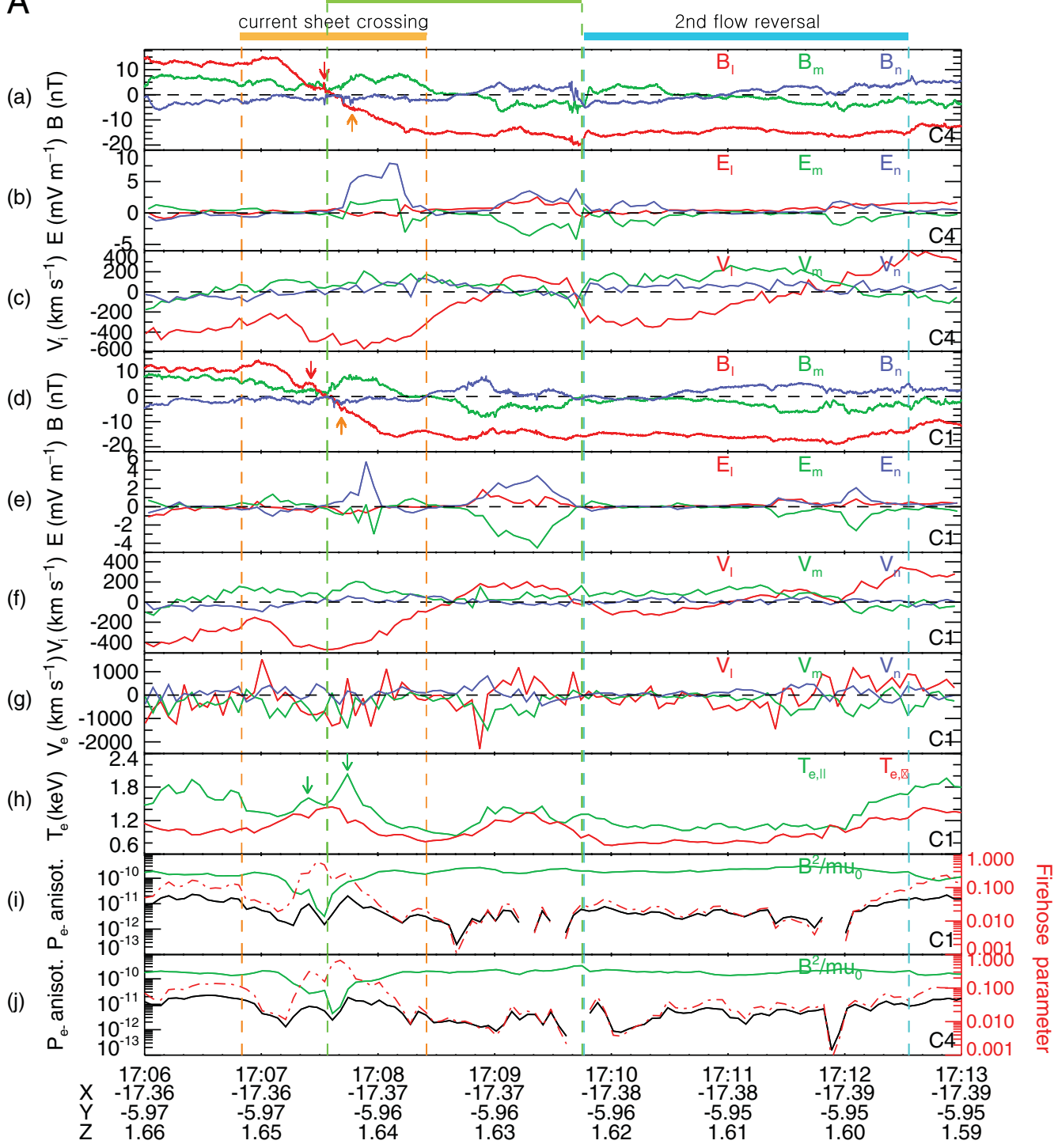

B

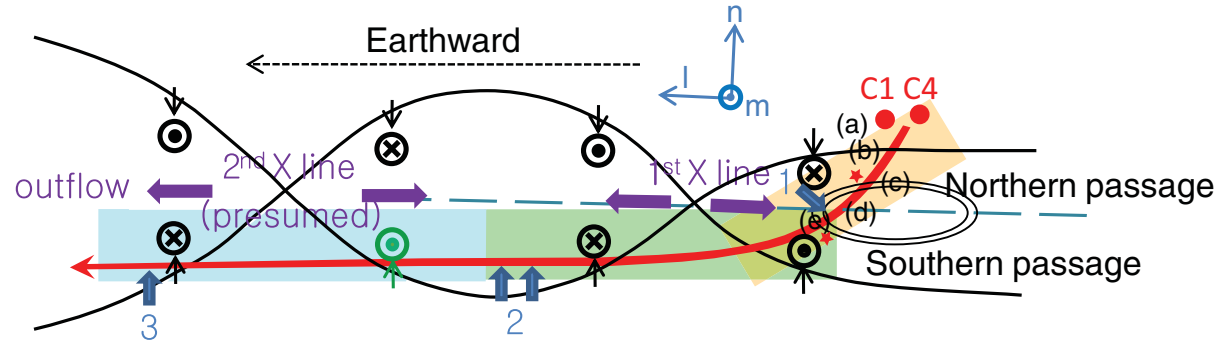

Figure 3. A. C4 (a)-(c), (j) and C1 (d)-(i) data in the current-sheet coordinates: (a), (d) magnetic field, $B_{l}$ (red), $B_{m}$ (green), and $B_{n}$ (blue); (b), (e) electric field, $E_{l}$ (red), $E_{m}$ (green), and $E_{n}$ (blue); ion (c), (f) and electron (g) velocity, $V_{l}$ (red), and $V_{m}$ (green), $V_{n}$ (blue); (h) electron parallel (green) and perpendicular (red) temperature, (i), (j) the electron pressure anisotropy $\left(p_{\|}-p_{\perp}\right.$, black) and the firehose parameter, $\left(p_{\|}-p_{\perp}\right) /\left(\frac{B^{2}}{\mu_{0}}\right)$ (red). B. The relative trajectory of $\mathrm{C} 1$ and $\mathrm{C} 4$ across the multiple $X$-line structures. Orange, green, and blue shades 
$\left(B_{m} \approx 6 \mathrm{nT}\right)$ prior to the first flow reversal represents a guide field. These data show consecutive Hall field features during two flow reversals (marked by green and blue bars).

To explore the reconnection substructure, a Walén test in the deHoffmann-Teller frame of reference was performed separately for ions and electrons. The Walén test locates rotational discontinuities that describe the current sheet layer under ongoing reconnection (Chanteur 1998). The test for electrons used the generalized Walén relation formula ((32) of Scudder et al. 1999) that takes into account the electron pressure anisotropy. For the northern passage, before $\mathrm{C} 1$ crossed the neutral sheet, only the electron Walén test showed a slope close to 1, as would be expected between jumps across an ongoing-reconnection boundary (a rotational discontinuity). On the other hand, the ions for the same time period showed a slope of 0.5, indicating that the ions are demagnetized and the spacecraft was in the IDR (Scudder et al. 1999). During the southern passage below the neutral sheet ('S'), neither the ions nor electrons satisfied the Walén test. The electrons did not show any significant correlation between $\left(V_{e}-V_{H T}\right)$ and $V_{\text {Alfven }}$, suggesting that north of the neutral sheet the spacecraft were mostly in the IDR and the electrons were still magnetized, while on the southern passage of the current sheet the spacecraft passed close to or were within the EDR since both ions and electrons were demagnetized.

Several papers have endeavored to identify the EDR using both observations and/or numerical simulations, and we only briefly cite a few here. Scudder et al. (2002) suggested that non-zero parallel electric fields supported by an electron pressure force on the scale of $\beta^{\frac{1}{2}} c / \omega_{p e}$ together with simultaneous observations of a super-Alfvénic parallel electron bulk flow, are signatures of being within the EDR. Mozer et al. (2005) proposed that an encounter with an EDR would be indicated by a combination of a non-zero parallel electric fields over a scale size of $d_{e}$, a perpendicular electric field exceeding the reconnection electric field, large Joule dissipation, electron beam acceleration, and a change of magnetic topology. Scudder and Daughton (2008) suggested that an EDR could be identified by the non-gyrotropy of the electron pressure tensor. Recently, Scudder et al. (2012) used data from the Polar spacecraft to identify an EDR at the Earth's magnetopause.

Although the EDR is, in general, difficult to identify solely using electron distribution functions, electron distribution functions can delineate the substructure of the current sheet where reconnection is occurring. Chen et al. (2008) and Wang et al. (2010) used signatures of temperature anisotropy and variations in electron velocity moments to identify magnetic islands and the scale size of an electron current layer. An anisotropic electron pressure with $p_{\|}>p_{\perp}$ within the reconnection region was first reported by Øieroset et al. (2002). This anisotropy was analytically and numerically accounted for Egedal et al. (2005), Egedal et al.

FIGURE 3. Continued.

depict the current sheet crossing, the first, and the second flow reversal, respectively. Red stars indicate a possible northern and southern traversal of the plasmoid structure embedded in the outflow region, associated with a transient enhancement of $B_{l}$, marked by red and orange arrows in panel A(a), (d) and electron temperature anisotropy $\left(T_{\|}>T_{\perp}\right.$, green arrows in panel $\mathrm{A}(\mathrm{d})$ ). During the second flow reversal (blue-shaded region) $\mathrm{C} 1$ did not observe tailward-side Hall fields (green symbols), although such fields were observed later by C4. Blue arrows, numbered 1-3 delineate when energetic electron fluxes were present; (a)-(e) indicate locations of the electron distributions shown in Fig. 4 (adapted from Fig. 1 of Hwang et al. 2013). 
(2008), Le et al. (2009), Egedal et al. (2010), Le et al. (2010) and Ohia et al. (2012) as resulting from electron trapping by magnetic mirror forces and parallel electric fields.

\subsection{1. $X$-points.}

The spacecraft trajectory relative to the reconnection geometry shown in Fig. 3(B) depicts the proximity to the $X$-line during Cluster's southern passage of the current sheet. Multi-spacecraft observations indicate that an $X$-line drifted close to the spacecraft, about $3.4 \mathrm{R}_{\mathrm{E}}$ earthward of the position where another $X$-line had been observed earlier. This observation provides important clues about the structure of reconnection layers and how $X$-lines develop and evolve. Comparison of the Hall magnetic and electric field geometry and the observed properties of energetic electron beams streaming along the separatrix between the Cluster spacecraft, indicates that the second $X$-line formed within 20 s of the observation of the first $X$-line. Repeated flow reversals and Hall field geometry suggest that the initial current sheet was unstable to the tearing-mode. In particular, the presence of a magnetic island embedded in the outflow region downstream of the first $X$-line appears to be coincident with the electron super-Alfvénic outflowing jet, suggesting that an instability in the elongated electron current sheet caused the formation of the plasmoid (Karimabadi et al. 2007).

Figure 4 shows detailed features of the electron distribution functions along the spacecraft trajectory in the vicinity of a reconnection $X$-line. The series delineates the structure of the reconnection current sheet. Near a reconnection $X$-line (and in the vicinity of the neutral sheet) there is a unique signature in the electron distribution function associated with traversing a sharp boundary in the electron current sheet. Hwang et al. (2013) identified the region to have a thickness of $0.72 d_{i}$ (corresponding to $29 d_{e}$ ) within which super-Alfvénic electron outflow (greater than the ion in-flow Alfvén speed) was observed. Slightly below the neutral sheet, Cluster observed asymmetric counter-streaming electrons with a loss of axisymmetry in the electron $\left(V_{\perp 1}, V_{\perp 2}\right)$ distribution functions over a thin boundary with a thickness of several $d_{e}$. This electron-scale transition layer was where both the ion and electron Walén test failed and the electron super-Alfvénic bulk outflow jets with high-energy electron beams were detected. Those phenomena suggest that the spacecraft traversed or skimmed the tailward edge of an elongated electron current layer, providing details of the substructure of the reconnection current sheet.

The present event exhibits significant differences from the reconnection event analyzed in and Chen et al. (2009), where strong electron temperature anisotropy with $T_{\|}>T_{\perp}$ was observed in the inflow regions, whereas the electron distributions in the exhaust were nearly isotropic. In contrast, for this event strong anisotropy was also observed in the exhaust and appears to be associated with the large-scale, elongated region of electron outflowing jets. This observation is consistent with a recent numerical study by Le et al. (2009), who showed that the temperature anisotropy develops due to electron trapping in the reconnection inflow. Subsequent simulations (Ohia et al. 2012; Le et al. 2013) predicted that the pressure anisotropy, approaching the firehose condition $\left(p_{\|}-p_{\perp}=B^{2} / \mu_{0}\right)$, can drive large-scale elongated electron current layers in the exhaust if the guide field is sufficient to maintain magnetized electrons. Figure 3(i), (j), indeed, shows that the firehose condition (reddotted) was approached near the neutral sheet where the elongated electron current layer is observed to be embedded. 
(a) C1 HEEA 2002-08-18/17:06:44.4

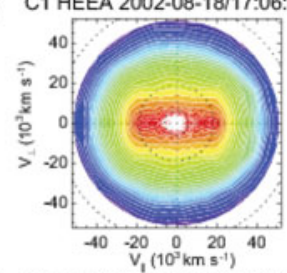

(b)

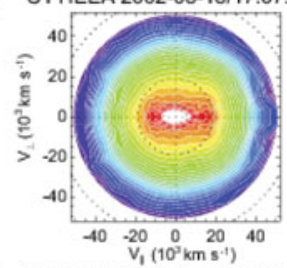

(c) C1 HEEA 2002-08-18/17:07:24.4

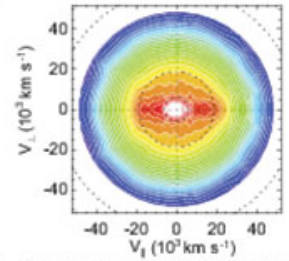

(d) C1 HEEA 2002-08-18/17:07:32.4

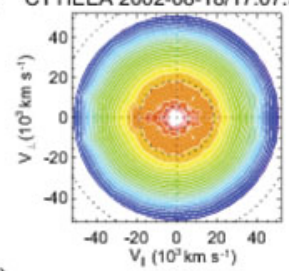

(e1) C1 HEEA 2002-08-18/17:07:37.4

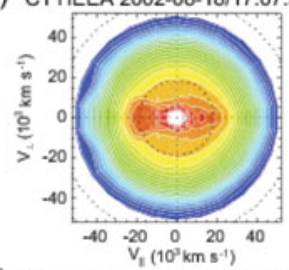

(e2) C1 HEEA 2002-08-18/17:07:41.4

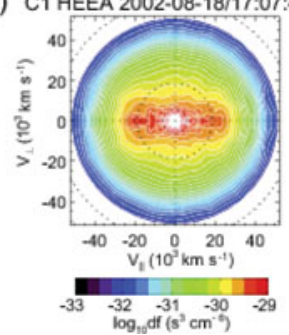

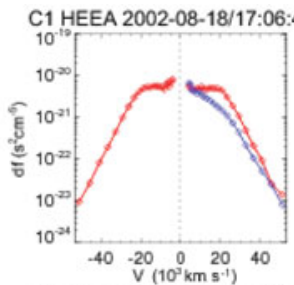

C1 HEEA 2002-08-18/17:07: 0.4

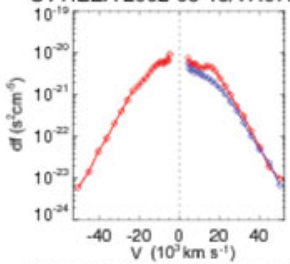

C1 HEEA 2002-08-18/17:07:24.4

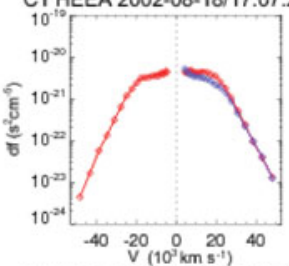

C1 HEEA 2002-08-18/17:07:32.4

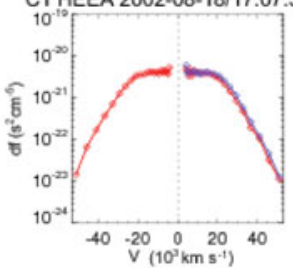

C1 HEEA 2002-08-18/17:07:37.4

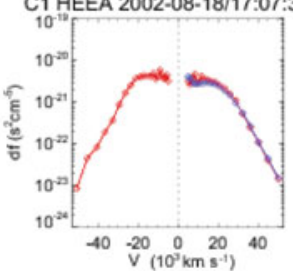

C1 HEEA 2002-08-18/17:07:41.4

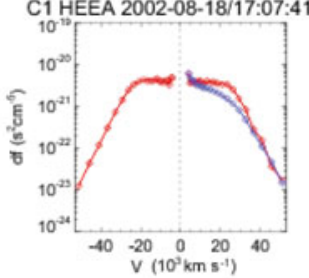

C1 HEEA 2002-08-18/17:06:44.4

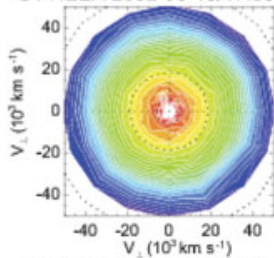

C1 HEEA 2002-08-18/17:07: 0.4

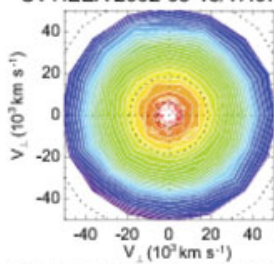

C1 HEEA 2002-08-18/17:07:24.4

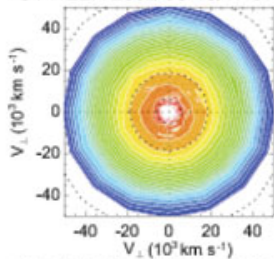

C1 HEEA 2002-08-18/17:07:32.4

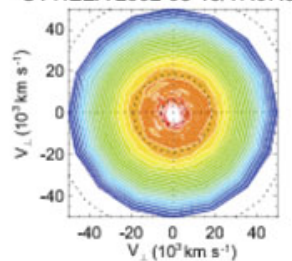

C1 HEEA 2002-08-18/17:07:37.4

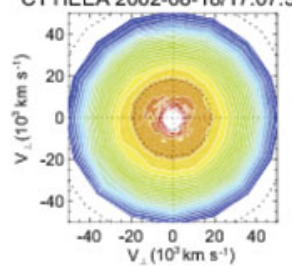

C1 HEEA 2002-08-18/17:07:41.4

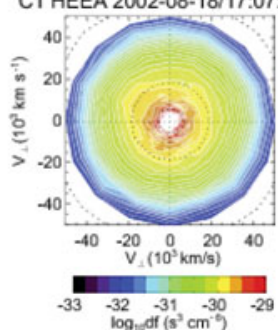

FIGURE 4. The electron distribution functions, $f$, at the time of crossing the inflow region at $\sim 1706: 44$ UT (a), a separatrix at $\sim 1707: 00$ UT (b), the northern-hemispheric boundary of a magnetic island downstream of the $X$-line at $\sim 1707: 24$ UT (c), the neutral sheet with a minimum of magnetic strength at $\sim 1707: 32$ UT (d), and slightly below the neutral sheet at $\sim 1707: 40$ UT $\left(\mathrm{e}_{1}\right.$ and $\left.\mathrm{e}_{2}\right)$. The first column shows $f\left(V_{\|}, \mathbf{V}_{\perp}\right)$, the middle one shows the reduced $f$ along the direction parallel (red) and perpendicular (blue) to the local magnetic field, and the last one shows the $f\left(V_{\perp 1}, V_{\perp 2}\right)$ from the 3-D electron distributions. Corresponding locations where in the current sheet frame $f$ are sampled are denoted along the spacecraft trajectory in Fig. 3B(a)-(e). Inner (outer) dashed circles overlaid in all 2-D distribution plots correspond to velocities of electron energies of 1 (10) keV. (From Fig. 4 of Hwang et al. 2013.) 


\subsubsection{Magnetic nulls.}

In geospace, global effects of reconnection are generally associated with X-points along separators (Dorelli et al. 2007a,b), however, in the solar corona large amounts of stored energy appear to be released at magnetic nulls (see e.g., Antiochos et al. 2002). Several regimes of non-ideal behavior and reconnection at magnetic null points have been hypothesized and modeled theoretically (Priest and Titov 1996; Pontin et al. 2005; Parnell 2007; Pontin and Galsgaard 2007; Pontin et al. 2007; Priest and Pontin 2009; Wyper and Jain 2010; Galsgaard and Pontin 2011a,b; Pontin et al. 2011), studied in the laboratory (Bogdanov et al. 1994; Lukin and Linton 2011), and inferred from coronal observations (Parnell 2007; Parnell et al. 2008).

Analyses of Cluster data at times when the orbital configuration formed a regular tetrahedron have provided confirmation of theories of the structure and evolution of current-carrying magnetic null points. Xiao et al. (2006), He et al. (2008a), Deng et al. (2009) and Guo et al. (2013) described an encounter with a magnetic null associated with reconnection in the Earth's magnetotail on October 1, 2001 during which time they attributed a nearby jet reversal and Hall magnetic field to reconnection near a null point. Later, He et al. (2008b) reconstructed the magnetic field around the same null and inferred that it was moving earthward. Xiao et al. (2007) discussed the same event in terms of a pair of spiral nulls of opposite polarity (also see, Runov et al. 2003; Cattell et al. 2005; Wygant et al. 2005). Based on the orientation of the spines and fans of the null points, Xiao et al. (2007) concluded that the nulls were connected by a separator. Furthermore, they were able to determine the velocity of the nulls and, consequently, deduced that the separator length was $\sim 860 \mathrm{~km}$, on the order of $\lambda_{i}$. Spectra constructed from magnetometer data taken in the vicinity of the separator (and within the IDR), identified the fluctuations as lower hybrid waves.

Using their fitting method, He et al. (2008a) extrapolated the surrounding magnetic topology and deduced that Cluster passed across the magnetic separator where it observed a peak in the electron density and $2 \mathrm{keV}$ electron beams jetting with speeds on the order of the electron Alfvèn speed. They concluded that the electrons underwent temporary trapping and acceleration from mirroring along the magnetic separator due the reconnection electric field and that the bidirectional electron beams were the source of the electrostatic solitary waves (ESWs)observed astride the separator. They deduced that the separator length was $\sim 880 \mathrm{~km}$.

For a nearby null pair observed on the same day, Deng et al. (2009) extrapolated a spiral null structure, where the spiral fan of one null wrapped around the spine of the adjacent null. They described enhancements in the electron flux up to $100 \mathrm{keV}$ near the null points and, based on FGM and STAFF data, identified whistler waves near the null points. The half-angle between the fan planes of the two nulls was close to the theoretical prediction of $19^{\circ}$ for the whistler wave expansion of the exhaust region, which they therefore associated with whistler-mediated reconnection near the null points. Using Cluster data from the same date, Guo et al. (2013) established the topology of two sets of null pairs and showed that while component reconnection applied to one pair, anti-parallel merging applied to the other pair.

Cluster observations on March 27, 2002 in Earth's turbulent, high-beta magnetosheath have provided a natural in situ test bed to explore localized null point reconnection. From about 9:30-11 UT, the spacecraft passed downstream of a quasi-parallel bow shock and into the magnetosheath along an orbit that passed near the southern cusp on the dawn side of noon (about 10:00 magnetic local time). Wendel and Adrian (2013) analyzed a pair of adjacent nulls captured between 
about 9:48:01 and 9:48:02 UT while the spacecraft tetrahedron was highly regular and the spacecraft spacing was about $100 \mathrm{~km}$. This is the same time period during which Retinò et al. (2007) found reconnection at numerous filamentary current sheets and Sundkvist et al. (2007) established the existence of a turbulent cascade (see Sec. 2.7.4). Wendel and Adrian (2013) derived the topological degree, or Poincaré index, and discovered magnetic nulls associated with many of the current filaments. The Poincare index is a measure of the number of roots, 'or magnetic nulls' in a region (Greene 1992). The origin, or root, in magnetic field space corresponds to a magnetic null in configuration space. Because the current layers crossed the spacecraft and the mean magnetic field was fluctuating on time scales of about $1 \mathrm{~s}$ or less, the analysis relied on electric fields outside the dissipation region - rather than on particle data to infer the neighboring plasma flows in the null rest frame. Wendel and Adrian (2013) concluded that the pair of nulls reflected a current structure and flow pattern that was consistent with torsional spine reconnection (Priest and Titov 1996; Pontin and Galsgaard 2007; Priest and Pontin 2009; Wyper and Jain 2010; Pontin et al. 2011).

The determinant and eigenvalues of $\nabla \mathbf{B}$ studied by Wendel and Adrian (2013) revealed that the null pair consisted of spiral $A_{s}$ and $B_{s}$ nulls. From the eigenvalues of $\nabla \mathbf{B}$ one can determine the nature of a spine and fan of a null. If all three eigenvalues are real, the null is either a type $A_{s}$ or type $B_{s}$ radial null. The eigenvalue $\lambda_{\gamma}$ that corresponds to the null spine $\gamma$ is of opposite sign to the other two eigenvalues, and its sign determines whether the spine points toward $\left(\lambda_{\gamma}<0\right)$ or away $\left(\lambda_{\gamma}>0\right)$ from the null. If one eigenvalue is real and the other two complex conjugates, then the null is either type $A_{s}$ or type $B_{s}$ and the sign of the real eigenvalue gives the orientation of the spine. Because the determinant of the matrix is the product of the eigenvalues, its sign specifies the polarity of the null. The topological degree $D$ is given by $D \equiv \sum_{n u l l s} \operatorname{sign}(\operatorname{det}|\nabla \mathbf{B}|)$ and represents a sum of like-type magnetic nulls in a given region (and will vanish if the region contains a pair of opposite nulls). A current $J_{\|}$along the spine produced skewed field lines in the fan plane, and a current $J_{\perp}$ perpendicular to the spine tilted the spine toward the fan. Figure 5 shows two views in the spine-aligned coordinate system of the superposed epoch of both nulls simultaneously, as they would be located at the time that null $B_{s}$ (placed at the origin) was captured. Arrows represent the fields measured by each spacecraft, where dark blue arrows denote spacecraft positions derived around null $A_{s}$, and red arrows denote spacecraft positions derived around null $B_{s}$. The top panel is a view along the normal to the $x-z$ plane, and the bottom panel a view along the spine of the plane perpendicular to the spine.

Transforming the EFW measurements of the electric field to the rest frame of the nulls yields the drift velocities about the nulls. Figure 6, from Wendel and Adrian (2013), shows the resulting perpendicular flows around null $A_{s}$. The top two panels show two different snapshots of flows around the null, and the bottom panel shows the components as a function of time. The uppermost panel is a view along a normal to the plane defined by the spine and the perpendicular current, and the middle panel is a view along the spine. As detected by $\mathrm{C} 1$, the bottom panel shows that the flow around null $A_{s}$ exhibited a distinct rotational pattern around the spine in the plane perpendicular to the spine. The view from the top panel suggests that the flow was actually rotating in the fan plane. The spacecraft was sufficiently distant from the null as to lie outside the diffusion region and, thus, the assumption $\mathbf{E} \cdot \mathbf{B}=0$ was valid. While the magnetic field is also rotational in this plane, the flow was still perpendicular to the field here. The flow became more rotational closer to the $z=0$ plane and closer to the spine (Wendel and Adrian 2013). 

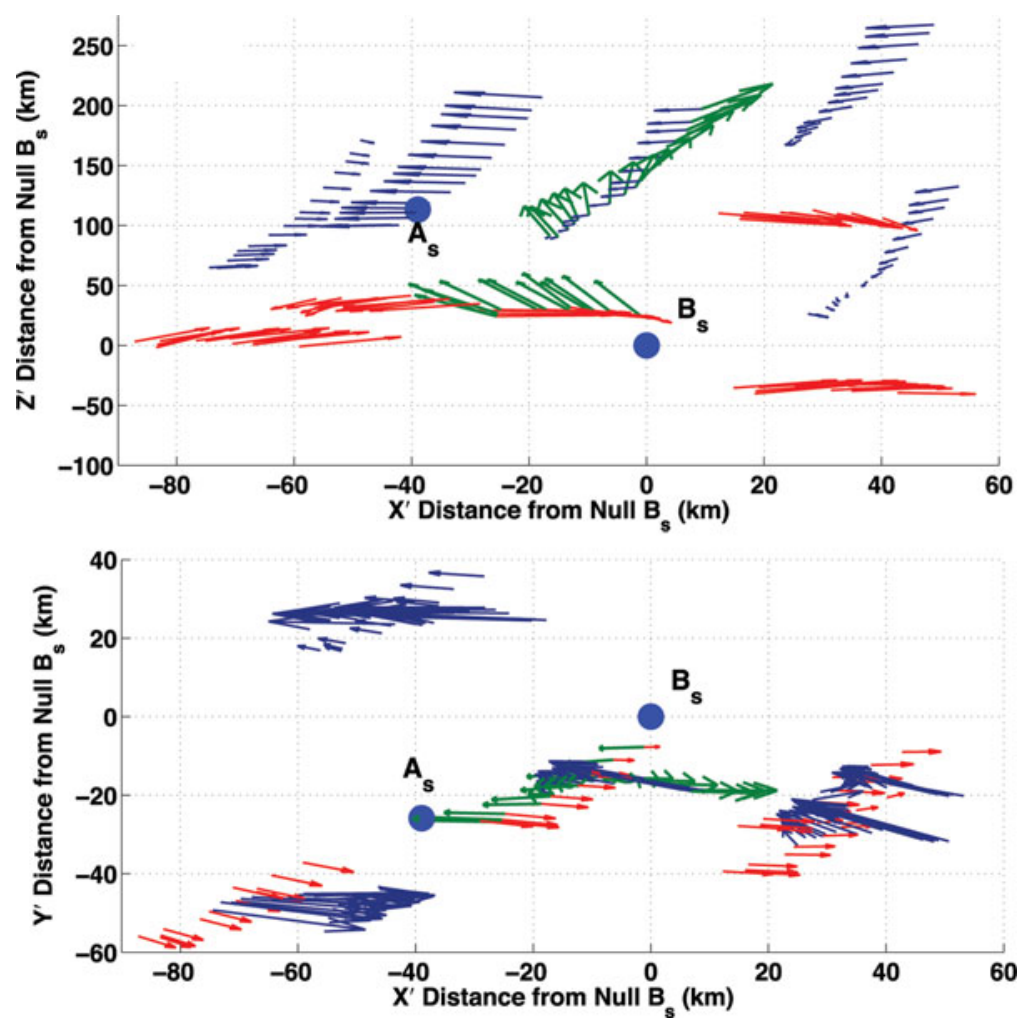

Figure 5. Two views of the instantaneous structure of the pair of nulls (blue circles) at their relative positions at the time null $B_{s}$ was captured. The field lines corresponding to $A_{s}$ are dark blue, and those belonging to null $B_{s}$ are red. The coordinate system is the spine-aligned system of null $B_{s}$. Green arrows signify the curlometer current (Sec. 1.4) at the position of the centroid. (Adapted from Wendel and Adrian 2013.)

Several authors (Titov and Hornig 2000; Pontin et al. 2004; Priest and Pontin 2009; Wyper and Jain 2010) have demonstrated that a diffusion region centered at the spiral null produces a rotational (spiral) flow around the spine. On field lines that have not passed through the diffusion region, both the electric field $\mathbf{E}$ and $\mathbf{v}_{\perp}$ are zero. The component of the drop in potential perpendicular to the null magnetic field lines as they exit the diffusion region induces a flow perpendicular to both the potential drop and to the magnetic field. Pontin and Galsgaard (2007) and Priest and Pontin (2009) explained the reconnection as a rotational slippage of field lines whereby the plasma elements above and below the diffusion region that share a given field line at time $t_{0}$ will, at a later time $t_{1}$, lie on different field lines. The spacecraft need not lie in or pass through the non-ideal region to detect flows of field lines that have engaged with the non-ideal region. Field lines that have not passed through the non-ideal region will have no rotational flow. Indeed, as Titov and Hornig (2000) have argued, the spiral flow is responsible for sustaining the null point.

Torsional spine reconnection is one form of the non-ideal null point behavior related to several similar forms of non-ideal behavior at current-carrying null points, such as torsional fan reconnection and spine-fan reconnection. The torsional spine and torsional fan reconnection do not transfer magnetic flux across topological domains and are therefore not generally associated with large amounts of energy 

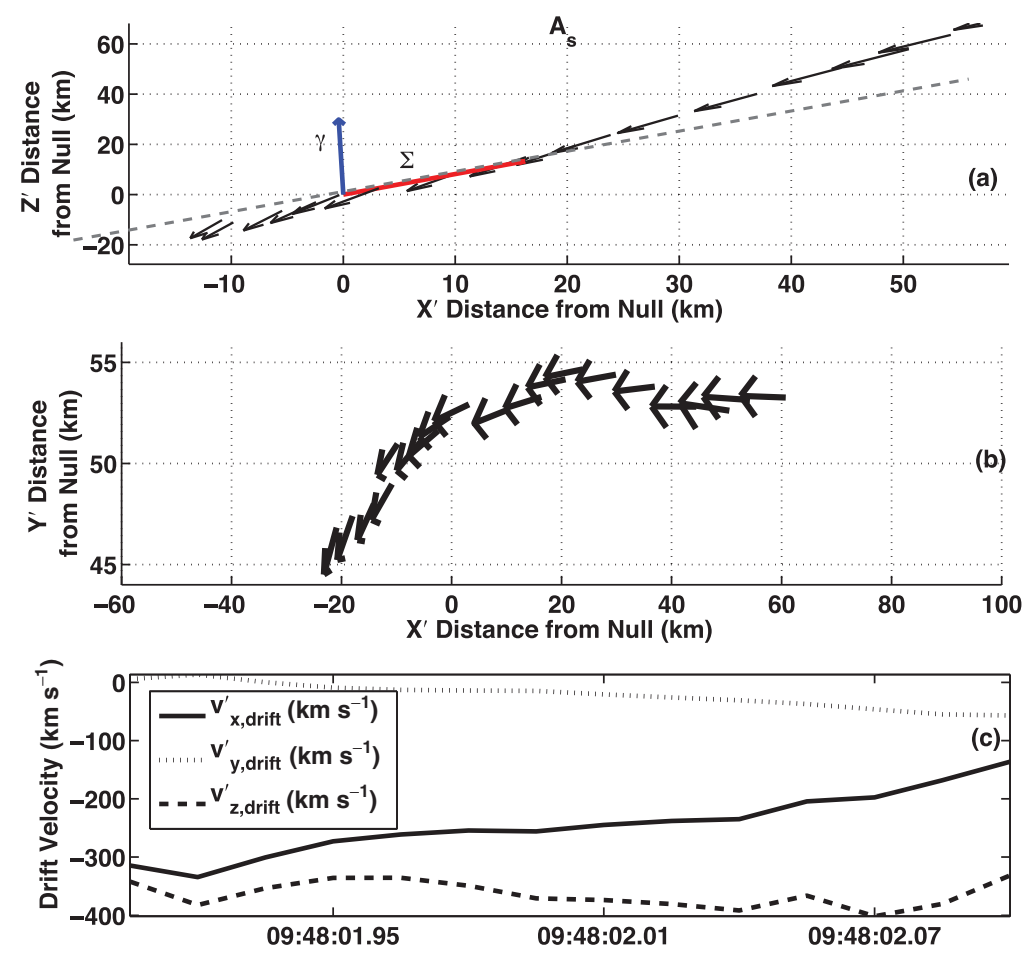

FIGURE 6. (a) View along the normal to the plane defined by the spine and the perpendicular current of the drift flows (black arrows) near null $A_{s}$ in the null rest frame. The blue and red arrows are the spine and fan plane, respectively. The spine and fan directions are determined at the position of the centroid at closest approach to the null. The dashed gray line is an extrapolation of the fan plane. (b) View of the same flows along the direction of the spine. (c) Magnitude of the components of the drift flows during the time interval of (a) and (b) in the spine-aligned coordinate system. (From Wendel and Adrian 2013.)

exchange. Rather, through non-ideal slippage of field lines around the nulls, they serve to dissipate energy. While regimes of null-point reconnection in general may not be an important source of energy exchange and flux transfer at the Earth, as mentioned above, some forms of null point reconnection are significant in the solar corona (Pontin et al. 2013). Cluster multi-point observations of null points in geospace present an opportunity to test simulation and theory in a natural environment.

Use of the four Cluster spacecraft has led to a significant progress in understanding the microphysics of magnetic reconnection, especially where reconnection occurs and where and how particles are accelerated. The morphology and general dynamics of the IDR have been studied in considerable detail. But there have been intrinsic limitations due in large measure to the time resolution of the particle instruments in particular and by the separation of the spacecraft. Even when the Cluster spacecraft were close, the time resolution of the particle instruments limited the physics that could be studied. With the forthcoming launch of MMS, many of these limitations will be ameliorated - the time resolution of the particle instruments will be much higher and the spacing of the spacecraft much closer, thus enabling a close investigation of the electron diffusion layer and more detailed analysis of the plasma dissipation and other kinetic effects. 


\subsection{Acceleration}

\subsubsection{Dipolarization fronts.}

DFs are a phenomenon commonly detected near the equatorial plane of the Earth's tail plasmasheet. They are characterized by sharp increases in the magnetic field component normal to the equatorial plane of the magnetosphere and are often associated with intense gradients of $B_{z}$. DFs are thought to result from magnetic reconnection in which the exhaust jets and entrained magnetic fluxes from the reconnection region pile up, forming a front of increased current-sheet-normal magnetic field (Hoshino et al. 2001; Hoshino 2005; Nakamura et al. 2009; Sitnov et al. 2009; Fu et al. 2013). DFs have drawn wide attention because they significantly affect the acceleration and transport of plasmas (see e.g., Sergeev et al. 2009; Zhou et al. 2009; Deng et al. 2010b; Ashour-Abdalla et al. 2011; Fu et al. 2011; Hwang et al. 2011a; Birn et al. 2013). DFs are also thought to play a significant role in populating the inner magnetosphere by transporting plasmas from the mid-tail (15-30 $\left.\mathrm{R}_{\mathrm{E}}\right)$ to the near-Earth (10-15 $\mathrm{R}_{\mathrm{E}}$ ) plasmasheet and, in some cases, into the inner magnetosphere (Delcourt et al. 1990; Delcourt 2002; Jones et al. 2006; Runov et al. 2009; AshourAbdalla et al. 2011). Cluster observations revealed both adiabatic and non-adiabatic particle energization including wave-particle interactions occurring at and around the fronts.

As an example, we describe the work of Hwang et al. (2011a), who reported Cluster observations of multiple DFs observed on August 15, 2001. The six DFs are indicated by magenta vertical lines numbered 1-6, at the top of Fig. 7. These DFs have a typical duration of 2-4 s in the spacecraft frame and a thickness of $900-1500 \mathrm{~km}$, which corresponds to the inertial length or gyroradius of $\mathrm{H}^{+}$ions. The common features across each DF are a sharp $B_{z}$ jump (Fig. 7(a)), a drop in the plasma density (Fig. 7(g)), a corresponding decrease in $\beta$ (Fig. 7(i)), a decrease in the plasma pressure and an increase in the magnetic pressure (Fig. 7(j)). Such plasma and magnetic-field variations across the DFs indicate that they carry an entropy-depleted flux tube. Figure 7 shows a decrease in the entropy calculated from the ion distribution function (black in Fig. 7(k)), or the flux-tube entropy parameter (red in Fig. 7(k)).

Numerous DF observations at the near-Earth plasmasheet by Cluster (e.g., Nakamura et al. (2002); Hwang et al. (2011a); Fu et al. (2011, 2012); Schmid et al. (2012); Hwang et al. (2014a)) and THEMIS (e.g., Runov et al. 2009; Sergeev et al. 2009; Deng et al. 2010b; Dubyagin et al. 2010; Zhou et al. 2010; Ashour-Abdalla et al. 2011) have shown that DFs predominantly propagate earthward along the radial direction. Occasionally, however, as was the case for DF 6, a DF is observed to propagate tailward. DFs are often embedded within fast earthward plasma flows known as bursty bulk flows (BBFs) (Angelopoulos et al. 1992, 1999). The BBFs follow 1-5 min after passage of a DF. The velocities of BBFs following behind DFs 1-3 are comparable to the Alfvén velocity, indicating that the multiple DFs might have resulted from bursty reconnection events or from continuous reconnection in which the reconnection rate fluctuated on the time scale of $\sim 3 \mathrm{~min}$, which corresponds to the occurrence rate of multiple DFs.

Particle-in-cell (PIC) simulations (Sitnov et al. 2009) and in situ observations by Cluster (Fu et al. 2013) have illustrated how DFs might arise from transient reconnection. Figure $7(\mathrm{~g})$ shows that the density ratio of $\mathrm{O}^{+}$to $\mathrm{H}^{+}$ions increases from $\sim 5 \%$ before the first DF to $\sim 20 \%$ at the end of the event. This indicates that a series of bursty reconnection events evolved from the plasmasheet to the lobe and that the repeated reconnection events may well have triggered multiple DFs. 


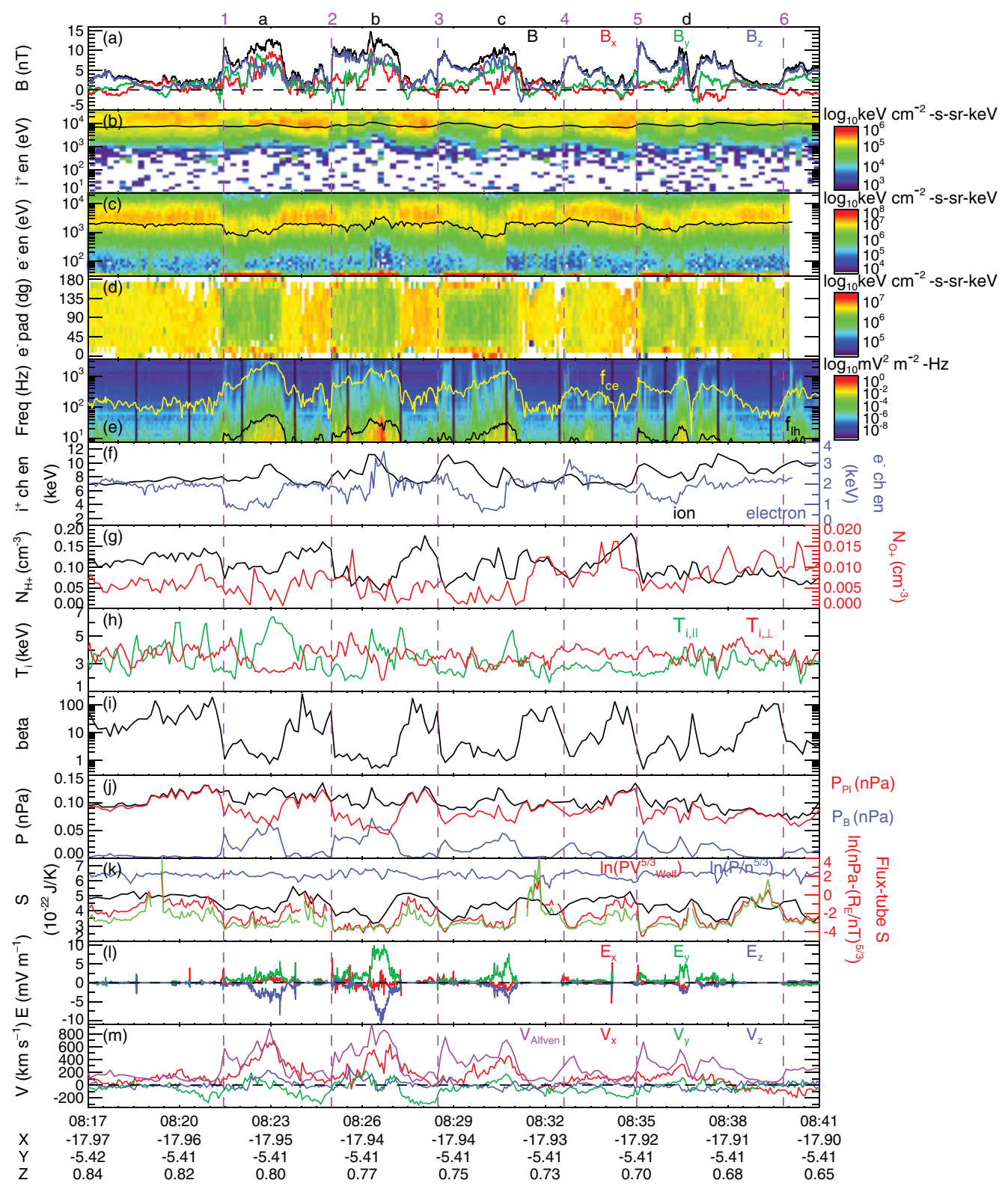

FIGURE 7. The field and plasma signatures of multiple dipolarization fronts observed by Cluster: (a) the magnetic field, $|\mathbf{B}|, B_{x}, B_{y}$, and $B_{z}$; (b), (c) the energy spectrograms of ions and electrons (the energy flux divided by the number flux is overplotted in black); (d) the electron pitch angle distribution; (e) the wave spectral power densities from the electric field (lower-hybrid frequency, $f_{l h}$ and electron cyclotron frequency, $f_{c e}$ are denoted as black and yellow curves.); (f), (g) the electron energy fluxes; (h) the ion parallel (green) and perpendicular (red) temperatures; (i) the plasma beta; (j) the plasma (red) and magnetic (blue) pressures, and the sum of the two (black); (k) the entropy from the ion distribution function (black), the 'flux-tube entropy' (red) $\left(P V_{B}^{\gamma}\right)$, derived using Wolf et al. (2006)'s formula for estimating the volume of a closed flux tube, $V_{B}$ (the green curve shows $1 / 3$ of the estimated $V_{B}$ ), and the specific flux-tube entropy, $P / n^{5 / 3}$ (blue); (1) the electric field, (m) the ion velocity (Alfvén velocity is shown in magenta). All parameters are plotted in GSM coordinates. (From Hwang et al. (2011a) Fig. 4.) 
Another possibility is that multiple DFs are related to the patchiness of near-Earth tail reconnection. Large-scale kinetic simulations (Ashour-Abdalla et al. 2011) show that multiple reconnection sites appear over the entire near-Earth tail current sheet for this event.

The behavior of DFs is not always simple. Hwang et al. (2014b) reported Cluster observations of a tailward-moving DF followed by an earthward-moving front indicating an origin in the near-Earth current sheet followed by tailward propagation, leading to mid-tail reconnection.

\subsubsection{Betatron and stochastic acceleration.}

The energization observed at DFs has been attributed to betatron and/or (first order) Fermi acceleration due to the local magnetic pile-up signature of DFs and the large-scale shortening of the magnetic field lines caused by radial convection of DFs (Birn et al. 2004; Ashour-Abdalla et al. 2011; Fu et al. 2011) in association with conservation of the first and second adiabatic invariants, respectively. The energization occurring at DFs can also arise from non-adiabatic processes, including wave-particle interactions (Deng et al. 2010b; Hwang et al. 2011a; Hwang et al. 2014a).

Global magnetohydrodynamic (MHD) and test-particle simulations have shown that adiabatic convection of particles from the mid-tail to near-Earth plasmasheet into the dipolar region of the night-side magnetosphere is associated with shortening field lines and increasing equatorial magnetic field strength. In the outer plasmasheet, as the distance from the Earth decreases, the field lines shorten rapidly while the equatorial field strength increases only slowly. In contrast, in the inner plasmasheet, the field line shortens slowly while the magnetic strength increases more rapidly. Hence, particles preferentially gain energy parallel to the magnetic field when they convect through the outer plasmasheet and gain perpendicular energy when they convect through the inner plasmasheet (Coroniti 1985). Indeed, DFs observed in the outer plasmasheet show that the pitch-angle distributions (Fig. 7(d)) of thermal electrons (energies $\lesssim 25 \mathrm{keV}$ ), are enhanced both parallel and anti-parallel to the background magnetic field. This type of distribution function is a signature of Fermi acceleration.

In general, when the time scale of the reconfiguration of magnetic topology is of the same order of (or shorter than) the gyroperiod and/or bounce period of particles, the acceleration process is likely to be non-adiabatic. DFs that lead to a rapid magnetic topology change can result in non-adiabatic acceleration that might explain the observed mass-dependent energization such as, e.g., the significant enhancement of energetic $\mathrm{O}^{+}$fluxes during geomagnetic storm time (Delcourt 2002; Ohtani et al. 2005; Fok et al. 2006; Keika et al. 2010).

DF events detected by THEMIS at $\sim-10 \mathrm{R}_{\mathrm{E}}$ often display enhanced electron fluxes at energies of about $1-100 \mathrm{keV}$ directly behind the front, while fluxes of lower energy electrons decrease. The increased populations peak at $90^{\circ}$ and/or $0^{\circ}$ and $180^{\circ}$ pitch angles, indicative of betatron and/or Fermi acceleration, respectively. In these events, energy fluxes of thermal electrons and ions vary consistently during DF crossings, i.e., more energetic populations of both ions and electrons are seen behind a DF. High-energy ions often show a gradual increase in energy flux ahead of a DF followed by a more rapid increase behind it, while the energy flux of highenergy electrons shows a sharp increase within a very limited region around DFs (Runov et al. 2011a,b).

On the other hand, Fig. 7 shows that for the multiple DFs observed by Cluster at $\sim-18 R_{E}$, the energetic populations were more prominent ahead of the DFs (for 
ions) and behind the DFs (for electrons). The observations explain how DFs act as a boundary separating a hot and dense plasma upstream (in the moving DF frame) and a cold and low density plasma region downstream. This is particularly true for observations made relatively close to the reconnection $X$-line (i.e., before the earthward convecting DF causes significant heating of the flux tube behind the DF). Thus, comparing DF observations from THEMIS and Cluster suggests that particle energization during DF crossings sensitively depends on where the observations were made.

Fu et al. (2011) focused on interactions between DFs and BBFs observed by Cluster and suggested that Fermi acceleration dominated inside a decaying DF (where the magnetic pileup region is behind the peak of the BBFs and, therefore, the $B_{z}$ pileup flux tubes are not compressed by BBFs perpendicular to B), while betatron acceleration dominates inside a growing DF where the magnetic pileup precedes the peak of the BBFs and the pileup flux tubes are compressed.

Wave-particle interactions occurring near DFs can result in non-adiabatic energization of plasmas. The common wave features near the DF (Sergeev et al. 2009; Zhou et al. 2009; Deng et al. 2010b; Hwang et al. 2011a) are the enhanced wave emissions close to the lower hybrid and electron cyclotron frequencies, or broadband wave emissions up to several times the electron cyclotron frequency. Lower hybrid drift waves are often generated by a diamagnetic current at a boundary having a gradient in plasma density and temperature, which are typically present at DFs. Lower hybrid waves can heat electrons preferentially perpendicular to the magnetic field (Daughton et al. 2004; Ricci et al. 2004). The electron cyclotron emissions have been linked to ring or shell electron distributions (Ashour-Abdalla and Kennel 1978; Hwang et al. 2011a). These wave emissions often appear to be coincident with the magnetic field maxima, being possibly related to changes in electron gyromotion occasioned by the increased magnetic strength. Huang et al. (2012) showed wave activity near the ion cyclotron frequency that arose from an Alfvén ion cyclotron instability driven by the cross-field ion drift in the front layer of a DF.

Behind the fronts, active wave emissions in the whistler frequency range are observed (Deng et al. 2010a,b; Hwang et al. 2011a; Huang et al. 2012; Hwang et al. 2014a; Viberg et al. 2014). Deng et al. (2010b) and Huang et al. (2012) attributed the generation of the whistler waves to the electron temperature anisotropy $\left(T_{\perp}>T_{\|}\right)$ caused by the pileup of the magnetic field lines, similar to the whistlers observed in the downstream region of the reconnection jet (Fujimoto and Sydora 2008). A statistical study using the Cluster measurements (Viberg et al. 2014) showed that the whistler mode waves are common in a vicinity of DFs, frequently within the magnetic flux pileup region behind a DF, in association with the electron temperature anisotropy. On the other hand, Hwang et al. (2011a), based on observations of strong parallel and antiparallel electron beams (instead of the perpendicular temperature anisotropy), speculated that the whistlers were excited by an electron beam instability. Hwang et al. (2014a), using both Cluster observations and linear theory, further explored wave enhancements behind DFs and found that two wave modes: a high-frequency beam mode and a low-frequency whistler mode, are associated with a localized electron beam. The generation of whistlers is associated with the electron beam components that can persist for a significant time before being quickly thermalized (Sauer and Sydora 2010). Considering the earthward motion of the DF flux tube, Fermi acceleration related to a shortening of the magnetic field lines contributes to preventing such electron beams from being rapidly thermalized, which can generate 
whistlers that can in turn energize particles, possibly leading to ion bulk heating. Hwang et al. (2011a) also reported observations of nonlinear electrostatic structures, e.g., electron solitary waves, within the flux tube behind a DF. Both electron cyclotron waves and nonlinear electrostatic structures can interact with the electron distribution and accelerate electrons stochastically to high energies (Farrell et al. 2003).

\subsection{Large-scale magnetic waves}

\subsubsection{Kelvin-Helmholtz waves.}

How plasma streaming along a boundary transfers matter, momentum, and energy across the boundary is important in many astrophysical situations. In the case of the solar wind and Earth's magnetosphere, when the interplanetary magnetic field (IMF) is northward, it has long been suggested that solar wind plasma enters the magnetosphere at the low-latitude boundary layer (LLBL) by exciting the Kelvin-Helmholtz instability (KHI)(see e.g., Hones et al. 1978). With Cluster, that phenomenon has now been studied in considerable detail.

Dungey (1954) first proposed that the KHI is important to the dynamics of the Earth's LLBL. In particular, a nonlinear stage of the KHI, i.e., large-scale, rolled-up vortices (KHVs) can facilitate entry of solar wind plasma into the magnetosphere via: (1) diffusive transport through the turbulent decay of the structure (Nakamura et al. 2004; Matsumoto and Hoshino 2004, 2006), (2) magnetic reconnection as the vortex motion generates a strongly stretched field (Otto and Fairfield 2000; Nykyri and Otto 2001; Nakamura et al. 2006, 2008), or (3) kinetic Alfvén waves through mode conversion from KH surface waves (Chaston et al. 2007). These processes can trigger plasma transport and mixing within or at the edge of rolled-up KHVs. Decaying KHVs ultimately form a broad mixing layer at the dawn and dusk flanks (Nakamura et al. 2006; Hasegawa et al. 2009; Cowee et al. 2009).

Recently, in-situ observations from Cluster, THEMIS, Geotail, Double Star, MESSENGER, and Cassini have indicated that the KHI appears capable of generating surface waves (e.g., Fairfield et al. 1990; Hasegawa et al. 2004; Nykyri et al. 2006; Fairfield et al. 2007; Volwerk et al. 2007; Taylor and Lavraud 2008; Boardsen et al. 2010; Masters et al. 2010; Cutler et al. 2011; Hwang et al. 2011b, 2012; Sundberg et al. 2012). KHW have also been identified in the magnetosheath (Walker et al. 2011b), the solar corona (Ofman and Thompson 2011), and the ionospheres of Mars and Venus (Pope et al. 2009). Cluster multipoint measurements have enabled us to identify KHVs (Hasegawa et al. 2004; Hwang et al. 2011b) and explore wave propagation, wave periods, boundary normals and the steepness of $\mathrm{KHW} / \mathrm{KHVs}$ (Owen et al. 2004; Nykyri et al. 2006; Foullon et al. 2008; Hasegawa et al. 2009; Foullon et al. 2010; Hwang et al. 2012).

Observations (e.g., Boardsen et al. 2010; Hwang et al. 2011b, and Fig. 8) and simulations (e.g., Walker et al. 2011a) have shown that KHW form and nonlinearly develop into large-scale vortices when the orientation of the IMF is opposite that of the planet's magnetic field at the magnetopause. However, for the opposite orientation, Kelvin-Helmholtz vortices (KHVs) were not expected since Miura (1995)'s first finding that the KHI at the magnetopause was most unstable during periods of northward IMF (because that orientation minimizes the magnetic tension force that stabilizes the KHI). However, under the assumption that the interface layer has zero thickness, the KH-instability condition for incompressible plasmas (Hasegawa 1975) shows no preference for northward over southward IMF.

The KHI becomes unstable when the shear velocity is greater than the local Alfvén speed along the direction of wave propagation and numerical studies of the KHI 


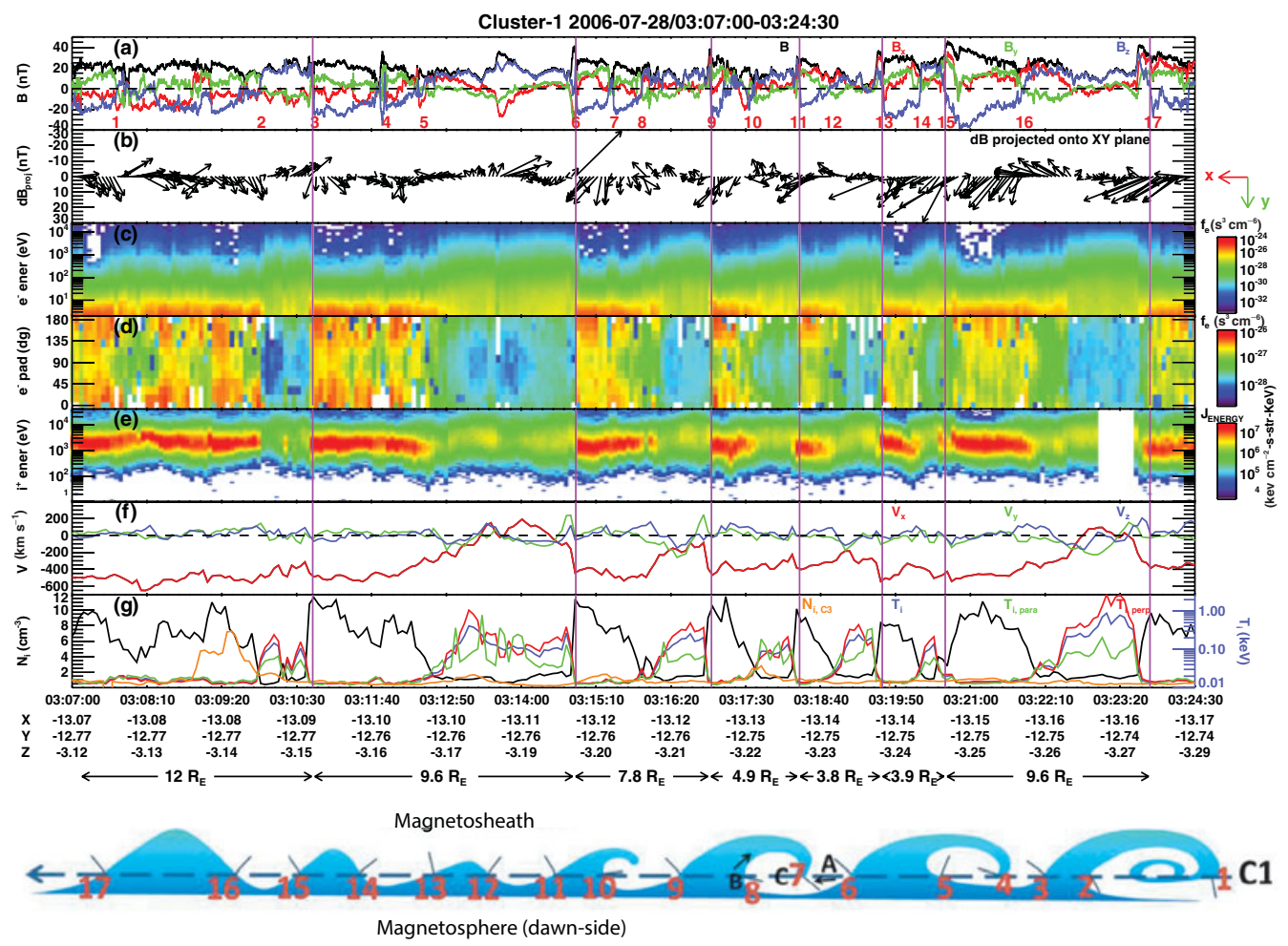

FIGURE 8. Cluster observations of $\mathrm{KH}$ surface waves at the dawnside flank of the tail magnetosphere during southward IMF conditions: (a) the $z$-component of the magnetic field, (b) deviations of the magnetic field components projected onto the velocity-shear plane ( $X Y$ plane) (c) the electron energy spectrogram, (d) the electron pitch angle spectrogram, (e) the ion energy spectrogram, (f) plasma density and (g) temperature. The bottom cartoon illustrates Cluster's trajectory relative to the $\mathrm{KH}$ wave trains. (Adapted from Hwang et al. (2011b).)

show that it is the thickness of the shear layer that controls the growth rate and that compressibility can be important (Miura and Pritchett 1982; Gnavi et al. 2006). However, the velocity shear is typically subsonic at the dayside magnetopause and the effect of the non-zero boundary thickness relaxes the KHI condition (Gratton et al. 2004). Increases in velocity shear and/or plasma density, or a magnetic field at both sides of the shear interface perpendicular to $\mathbf{k}$ lowers the KHI threshold. Thus, both northward and southward IMF can be unstable to tailward propagation of KHW along the LLBL of the magnetosphere.

Figure 8 shows the first observation of well-developed KHW during southward IMF (Hwang et al. 2011b). $B_{z}$ perturbations (Fig. 8(a)) indicate that Cluster encountered the magnetosheath (negative $B_{z}$ ) and the magnetospheric region (positive $B_{z}$ ), repeatedly. The two regions are also characterized by a large population of low-energy particles, high density, and low temperature during negative $B_{z}$ (magnetosheath) and a large population of high-energy particles, low density, and high temperature during positive $B_{z}$ (magnetosphere). The repeated observation of such correlations between the magnetic field and plasma signatures indicates that the scale size and evolutionary phases among these $\mathrm{KH}$ wave forms (separated by vertical dashed lines) are highly 
variable during the $\sim 20 \mathrm{~min}$ of the event. Four-spacecraft analyses revealed that the first half of the KHW trains represents nonlinear KHVs, while the other half were linear waves. Based on these observations, Hwang et al. (2011b) emphasized that southward IMF typically generated dynamically active subsolar activity that led to KHW evolution that had intermittent and irregular features, making it difficult to observe well-developed KHV when the IMF was southward. Global MHD simulations (Kuznetsova et al. 2008) demonstrated the dynamical complexity of KHV during southward IMF.

On January 12, 2003 Cluster observed the generation and evolution of KHW under variable IMF conditions (Hwang et al. 2012). This was the first in-situ observation of KHW in the high-latitude magnetopause near the northern duskward cusp under strongly dawnward IMF conditions. The near-cusp magnetopause is rarely unstable to KHI mainly due to strong geomagnetic field in that region. However, the magnetic configuration across the boundary layer near the northern duskward magnetopause during dawnward IMF is similar to that at the dayside LLBL under northward IMF in that the magnetosheath and magnetospheric fields across the boundary layer constitute the lowest magnetic shear, and the tailward propagation of the KHW is perpendicular to both fields. These observations indicate that KHW may be common over the entire surface of the dayside magnetopause for a wider range of IMF orientations than had been thought previously, providing a continuous conduit for the solar wind to enter the magnetosphere.

\subsection{Small-scale \& high frequency plasma waves}

\subsection{1. $A K R$.}

The Cluster mission has unique capabilities for studying AKR in ways never before possible. The physical processes that generate AKR appear to be common to many astrophysical environments, in particular, the magnetospheres of magnetized bodies. Although the energies involved in AKR are not particularly high $(\sim 1-10$ $\mathrm{keV}$ ), relativistic effects are, nonetheless, of fundamental importance. In this section, we highlight a few key results to illustrate the significant contributions of Cluster's multi-spacecraft observations to understanding the physics of AKR.

Prior to the launch of Cluster, observations and theoretical modeling had shown that AKR was most likely generated by electrons moving along active auroral magnetic field lines and through electron density cavities (e.g., Delory et al. 1998; Pritchett et al. 2002). Freely propagating electromagnetic radiation can be produced near $f_{c e}$ by the (relativistic) cyclotron maser instability (CMI) (Wu and Lee 1979; Fung and Viñas 1994; Pritchett et al. 2002). Because there was no way to establish connections between separate observations near the source and observations at remote locations, it was never possible to associate the remote AKR observations with specific source regions. The tetrahedron formation of Cluster above the source region, as illustrated in Fig. 9, allows for simultaneous observation of the same AKR burst from slightly different vantage points. This has enabled both an accurate determination of the source location and led to a comprehensive characterization of the AKR beaming pattern (Mutel et al. 2003, 2004, 2008).

As illustrated in Fig. 9, AKR signals emanating from a (single) compact source at $\mathbf{R}_{s}$ and detected by two different satellites, at $\mathbf{R}_{1}$ and $\mathbf{R}_{2}$, ought to be correlated, but perhaps time-shifted from one another due to different propagation distances. The difference in propagation times, which can be determined by cross-correlation 


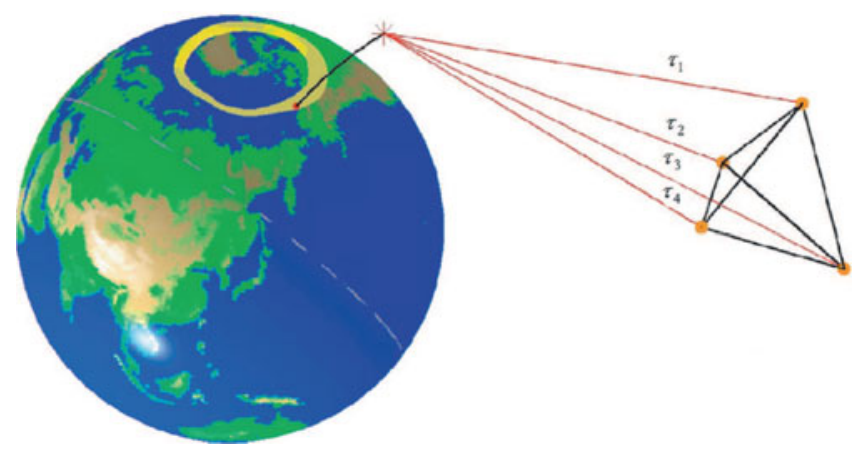

FIGURE 9. A schematic of nearly simultaneous detection by the four Cluster spacecraft of AKR emanating from the same radiation source located above the Earth along an active auroral field line (from Mutel et al. 2004).

analysis (Mutel et al. 2004), is given by assuming rectilinear propagation

$$
\boldsymbol{\tau}_{12}=\boldsymbol{\tau}_{1}-\boldsymbol{\tau}_{2}=\frac{\mathbf{R}_{1}-\mathbf{R}_{s}}{c}-\frac{\mathbf{R}_{2}-\mathbf{R}_{s}}{c} .
$$

It then follows that for any given source location, a signed sum condition $\boldsymbol{\tau}_{i j}+$ $\boldsymbol{\tau}_{k i}+\boldsymbol{\tau}_{j k}=0$ exists for every triplet $(i, j, k=1-4)$ of satellite measurements. Because the Cluster constellation provides six independent baselines for calculating propagation time differences according to (2.1), the AKR source location (with only three unknowns) is over-determined and can be localized to within a volume by minimizing the signed sum to within the timing measurement uncertainties (Mutel et al. 2003, 2004). Using this technique Mutel et al. (2004, 2008) determined the intrinsic properties of AKR sources and beaming characteristics. They found that AKR is generally emitted from individual sources located in the nightside auroral zone (see Fig. 3 in Mutel et al. 2004), consistent with previous statistical results.

Whether the AKR emission cone is hollow (Benson and Calvert 1979; Calvert 1981), or filled (Green et al. 1977; Green and Gallagher 1985) has been debated since the early days of AKR research. The debate hinges upon the AKR emission process and the plasma structure surrounding the source. Using the fact that to obtain a valid source location, at least three (angularly separated) spacecraft need to be illuminated by the same AKR beam, Mutel et al. (2008) determined that AKR emission beams are intrinsically narrow in latitude. In particular, they concluded that an AKR emission beam is confined to a plane (to within $\sim \pm 20^{\circ}$ ) containing the active auroral field line threading its source and tangent to the latitudinal circle at the source (Fig. 10). This beaming pattern is consistent with the FAST satellite measurements of wave electric fields showing that the 'along-track' electric fields are always stronger (by a factor of 100) than the 'cross-track' fields (Pritchett et al. 2002). Mutel et al. (2008) concluded that the tangent plane pattern appears to be independent of AKR frequency $(>125 \mathrm{kHz}$ ), and is not consistent with the hollow emission cone scenario of Calvert (1981). A filled cone pattern could result if there were contributions from multiple distributed source elements, although a random sum of multiple sources would not necessarily result in a systematic variation of cone angle with AKR frequency (Green et al. 1977; Green and Gallagher 1985).

Cluster observations have improved our understanding of the fine structure of AKR that was first observed by ISEE 1 and 2 (Gurnett et al. 1979; Gurnett and Anderson 1981) and Dynamics Explorer 1, Galileo, and Polar (Menietti et al. 1996, 2000). 


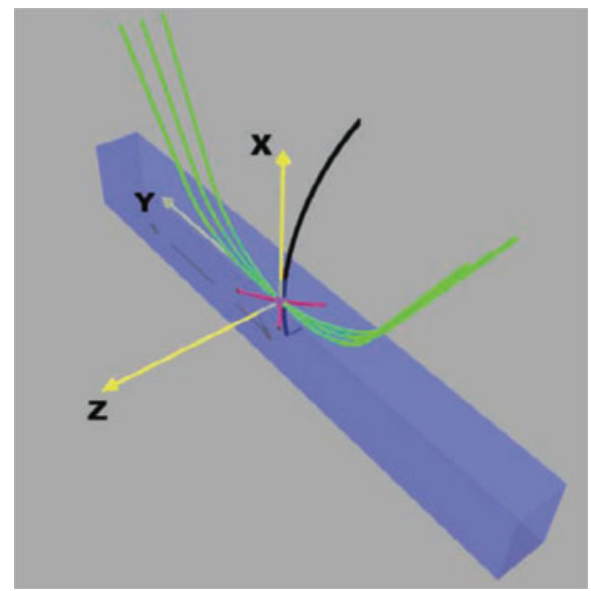

FIGURE 10. AKR emission (green curves) is confined (to within $\pm 20^{\circ}$ ) to a locally tangent $(X-Y)$ plane at the source region. The $X$-axis is along the source magnetic field direction. The $Y$-axis is along the longitudinal direction, and $Z$ gives the outward normal direction (adapted from Mutel et al. 2008).

Fine structure, or striated, AKR (SAKR) consists of narrowband drifting spectral features with wave frequency increasing, remaining relatively constant, or decreasing with time. Often appearing over a broadband-AKR, the SAKR tends to form groups (Mutel et al. 2006). Cluster observations indicated that the bandwidth of SAKR (at $125 \mathrm{kHz}$ ) can be quite small, $\lesssim 50 \mathrm{~Hz}$, which is much narrower than the bandwidth $(<1 \mathrm{kHz})$ determined previously from ISEE observations. The narrow bandwidths and negative frequency drifts of individual SAKR bursts observed by Cluster suggest that SAKR sources are compact $(\approx 1 \mathrm{~km}$ extent along the auroral field line $)$ and are moving upward along the field with speeds comparable to the ion-acoustic speed. These observations led Mutel et al. (2006) to conclude that SAKR sources are upward propagating small-scale ion holes or ESWs that can modify the electron distributions in AKR source regions to produce enhanced narrowband emissions over the regular broadband AKR background.

Mutel et al. (2006) analyzed 651 individual SAKR bursts and determined statistically that an SAKR beam at $125-135 \mathrm{kHz}$ has a FWHM width of $5^{\circ}$ (solid angle $\Omega=0.006 \mathrm{sr}$ ), which is much narrower than the ensemble-averaged AKR emission cone sizes of $4.6 \mathrm{sr}$ at $178 \mathrm{kHz}$ and $3.3 \mathrm{sr}$ at $100 \mathrm{kHz}$ reported by Green and Gallagher (1985). While it may be reasonable to regard commonly observed AKR generally as ensembles of emissions from distributions of 'elemental radiators' (Pottelette et al. 2001), it is not clear if all AKR is comprised of only SAKR bursts. The observations reported by Mutel et al. (2006) showed that SAKR appeared as negatively drifting features $(d f / d t<0)$ superposed on regular broadband AKR background. These observations suggest that the SAKR-producing structures are ion holes moving upward through coeval AKR sources, although this scenario does not account for the upward $(d f / d t>0)$ or nearly constant $(d f / d t \sim 0)$ spectral striations seen in earlier observations (Gurnett et al. 1979; Gurnett and Anderson 1981; Menietti et al. 1996, 2000). If AKR consists solely of SAKR elements, then the auroral field lines within the night-side upward field-aligned current region would need to be densely populated with ion hole structures to sufficiently account for the overall AKR emission cone observations. 


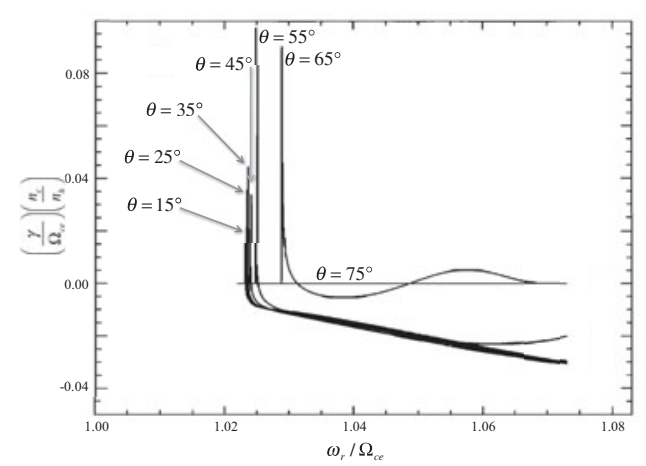

FIGURE 11. Linear growth rate due to a horseshoe distribution as a function of normalized frequency $\omega_{r} / \omega_{c e}$ for various wave normal angles, $15^{\circ}-75^{\circ}$, at $10^{\circ}$-intervals (adapted from Fung and Viñas 1994).

Due to the presence of an electrostatic field-aligned potential drop along auroral field lines, the combined distribution of the energetic precipitating and mirroring electrons can form a horseshoe distribution. Fung and Viñas (1994) showed that a drift-loss cone or horseshoe distribution with finite thermal spread is not only more unstable than the simple loss cone typically employed in CMI studies, it can also produce radiation consistent with AKR signatures. Unlike the CMI that produces radiation mostly perpendicular to the magnetic field (e.g., Mutel et al. 2007), Fung and Viñas (1994) showed that obliquely propagating waves can, in fact, be more unstable. In addition, waves propagating in the same oblique direction tend to be produced within a narrow bandwidth (spike). The spike becomes narrower as the wave normal angle becomes smaller (cf., Fig. 11). Assuming a spike bandwidth of $\Delta \omega_{r} / \omega_{c e} \sim 0.01 / 50$, then for $f \approx f_{c e} \sim 125 \mathrm{kHz}$ we have $\Delta f \sim 25 \mathrm{~Hz}$, quite consistent with the SAKR bandwidth $(\lesssim 50 \mathrm{~Hz})$ seen by Cluster. Figure 11 shows that the central frequencies of the spikes are also ordered by their wave normal angles. Such ordering implies that a specific sense of frequency drift (positive or negative) would result from the latitudinal motions of either or both the satellite and a group of emission cones, causing either rising or falling striations. Figure 11 also indicates that waves generated at larger wave normal angles can have secondary peaks of growth that have much broader bandwidths, which could explain the frequent presence of broadband AKR together with SAKR.

Other mechanisms have been proposed to explain SAKR. Pottelette et al. (2001) presented a theory based on electron holes to explain typical or random fine structure of AKR. Menietti et al. (2006) pointed out that electrons holes are more abundantly observed in downward current regions, so they would be less likely to be responsible for generating AKR emissions, which is thought to be produced in upward fieldaligned current regions. Mutel et al. (2006) proposed that ion holes, rather than electron holes or tripolar structures, are the source of AKR ordered fine structure. Analyzing Cluster WBD observations of ordered AKR fine structure and electron distributions taken by the Polar HYDRA instrument near magnetic conjunction, Menietti et al. (2006) found that the unstable electron horseshoe distributions in/near the AKR source region can also produce electromagnetic ion cyclotron (EMIC) waves. They proposed that ordered AKR fine structures can be stimulated by upward propagating EMIC waves. 


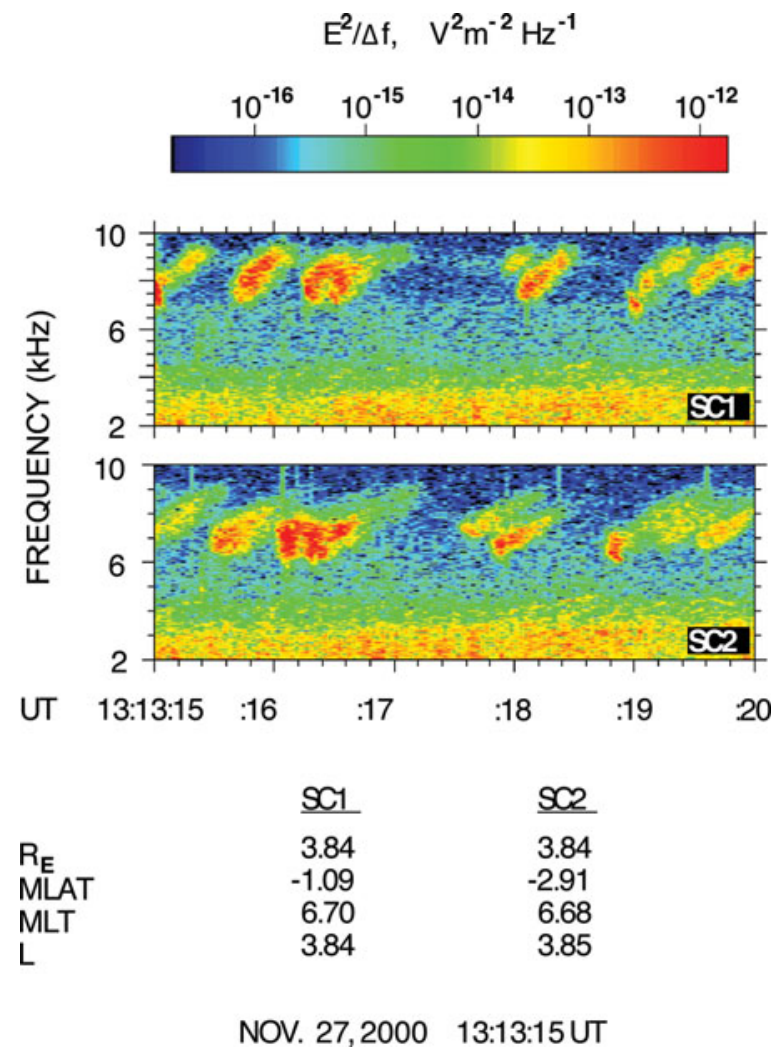

FIGURE 12. Spectrograms of the correlated chorus emissions from two Cluster spacecraft near the geomagnetic equator over $5 \mathrm{~s}$. The correlated emissions are $1 \mathrm{kHz}$ higher in frequency on C1 than on C2. (From Gurnett et al. 2001.)

\subsubsection{Chorus, electron interactions, and acceleration.}

One of the most prolific areas of research undertaken with data from the Cluster mission is that of whistler mode chorus waves in the inner magnetosphere. ELF/VLF chorus emissions are very intense electromagnetic whistler mode waves that are naturally and spontaneously excited near the magnetic equatorial plane outside the plasmasphere during periods of magnetic disturbance. It is now widely thought that chorus is generated by a nonlinear process (Nunn et al. 1997; Trakhtengerts et al. 2007) involving the electron cyclotron resonance of whistler-mode waves with energetic electrons (Andronov and Trakhtengerts 1964; Kennel and Petschek 1966). Until the launch of Cluster, experimental research on chorus waves had nearly ceased. Many new results have now been obtained, made possible by use of Cluster multispacecraft, multi-point measurements. Some of the early Cluster results are detailed in a review by Santolík (2008). Below we highlight some of these results, as well as those which have been published since that time.

Propagation. The first unexpected result regarding chorus waves is related to their propagation. For example, Gurnett et al. (2001) found that correlated chorus elements observed by WBD across two spacecraft had significant frequency variations $(\sim 1 \mathrm{kHz})$, indicating that the frequency of the wave packets might have evolved as the wave propagated to the two spacecraft (see Fig. 12). This character of the chorus waves could not have been uncovered without multi-point measurements. Inan et al. 
(2004) and Platino et al. (2006) carried out follow-up studies and concluded that this frequency difference was "a natural outcome of the dependence of the whistlermode refractive index on the wave normal angle between the wave vector $\mathbf{k}$ and the static magnetic field $\mathbf{B}_{o}$, and the rapid motion of highly localized source region(s) of chorus" of 400-1700 km in extent along the field line, but less than $100 \mathrm{~km}$ transverse to the magnetic field. The fluctuations were moving at speeds of 20000-25000 $\mathrm{km} \mathrm{s}^{-1}$. Chorus wave packets emanating from the localized regions propagated to two spacecraft at different frequencies were detected in the differential Doppler shift between the spacecraft. Inan et al. (2004) and Platino et al. (2006) used these frequency differences to study the motion of the sources. Their results provide the first experimental evidence that the sources generating discrete chorus emissions were in rapid motion.

Chum et al. (2007) used multi-point data to show that if a non-moving or quasi-static source emitting waves in a relatively narrow interval of wave normal angles in both the wave normal angle and frequency during the generation of a single chorus element, then the resultant radiation can produce the observed frequency shift of chorus wave frequencies. In another study of obliquely propagating chorus, Chum et al. (2009) used WBD and STAFF to show remarkable frequency differences and time shifts between the corresponding elements of lower band chorus. "Surprisingly, the spacecraft located closer to the equator systematically received the corresponding chorus elements later than the spacecraft located at higher magnetic latitudes. Owing to the orbit, the spacecraft located closer to the equator were at lower $L$ shells. The time shifts and frequency differences depended almost linearly on the perpendicular distance between the spacecraft." The work showed that chorus sources moving across the magnetic field lines are reasonably consistent with the observations and the authors "concluded that the transverse motion of the chorus sources is a consequence of a feedback between the oblique waves and counter-streaming electrons during nonlinear cyclotron interactions." Breneman et al. (2007) and Breneman et al. (2009) explored the chorus element frequency variation by studying the chorus wave characteristic frequency/time variation that is a combination of frequency separation by propagation dispersion and a time-dependent source frequency emission drift. They used a cross-correlation technique to compare data from multiple Cluster spacecraft to quantify the frequency variation of the chorus emissions due to the dispersion. Comparison of the data crosscorrelations with simulated cross-correlations (using ray tracing methods) allowed them to determine the time-dependent source frequency emission drift. They found that the waves with frequencies above and below $f_{c e} / 2$ on the magnetic field line of the spacecraft were emitted in a broad spectrum of wave normal angles (where here $f_{c e}$ is the cyclotron frequency at the equator). They also found that there is some preference for the lower band waves to be emitted near the Gendrin angle and at earthward-pointing wave normal angles of between -20 to $-30^{\circ}$. The time-dependent source frequency emission drift that they found for the events studied ranged from 1 to $20 \mathrm{kHz} \mathrm{s}^{-1}$. This rate accounted for at least $2 / 3$ of the chorus frequency/time variation with the rest being due to propagation dispersion.

Source Region. Studies of the source region of the chorus waves have yielded significant new information about both the wave generation region and about the properties of the waves themselves. The initial study (Santolík and Gurnett 2003) focused on the generation region found that cross-spacecraft correlation for stormtime chorus is significant throughout the range of separation distances of $60-260 \mathrm{~km}$ parallel to the magnetic field and $7-100 \mathrm{~km}$ in the perpendicular plane. There were two follow-up studies: In the first, Santolík et al. (2004) used measurements from 
WBD from the geomagnetic storm on 31 March, 2001 and found that the observed spatio-temporal variations of the direction of the Poynting flux manifest a consistent pattern: the central position of the chorus source fluctuates on time scales of minutes within 1000-2000 km of the geomagnetic equator. In the second, Santolík et al. (2005) used WBD observations from the geomagnetic storm of 18 April, 2002 and determined that the global central position of the source region fluctuates within a few thousands of kilometers at a typical speed of the order of $100 \mathrm{kms}^{-1}$. This random motion is most probably associated with the generation mechanism of chorus. Another major finding with regard to chorus waves was made by Horne et al. (2005) using WBD and data from the Palmer station in Antarctica. The study concluded that chorus waves may have been responsible for the acceleration of electrons in the Van Allen radiation belts during the major Halloween storms of October and November 2003. On the basis of the analysis of this rare event where the outer radiation belt was depleted and then re-formed closer to the Earth, the long established theory of acceleration by inward radial diffusion was proven to be inadequate. The electrons were more likely to have been accelerated locally in the inner $L$-shell region by electromagnetic waves (chorus) at frequencies of a few kilohertz. Horne et al. (2005) determined that "wave acceleration can increase the electron flux by more than three orders of magnitude over the observed timescale of one to two days, more than sufficient to explain the new radiation belt".

Santolik et al. (2008) analyzed observations recorded by the WBD instrument of a night-side chorus event during geomagnetically disturbed conditions. They investigated the central position of the chorus source that had been previously estimated from Poynting flux measurements. Their results indicated a lower occurrence probability for lower frequencies in the vicinity of the central position of the source compared to measurements recorded closer to the outer boundaries of the source. This is in agreement with the backward wave oscillator (BWO) theory that attributes chorus generation to an absolute instability of whistler-mode waves in the presence of a step-like velocity distribution of energetic electrons. Kozelov et al. (2008) also studied the motion of the source region of chorus emissions. Comparison of WBD and magnetic field data showed that the chorus source remains related to the magnetic field minimum, although the position of this minimum can vary rather strongly during periods of enhanced geomagnetic activity. These results are also consistent with the BWO model. While most of the quasi-linear and nonlinear theoretical studies assume that the waves propagate parallel to the terrestrial magnetic field, Santolík et al. (2006) had previously concluded that chorus or chorus-like waves, generated with highly oblique wave vectors in a source region located close to the geomagnetic equator outside the plasmasphere (at radial distances above $5 R_{E}$ ), are very likely the source emissions of a large class of low-altitude wave phenomena, namely ELF hiss. Using ray tracing, (Bortnik et al. 2008, 2009) later came to a similar conclusion. Santolik et al. (2009) presented a case of oblique chorus propagation using Cluster WBD where they showed that "chorus in the source region can be formed by a succession of discrete wave packets with decreasing frequency that sometimes change into shapeless hiss", occurring at the same time in the entire source region. Thus, the generation of chorus and hiss can be very complex and is not yet fully understood.

Trakhtengerts et al. (2007) used WBD to reveal for the first time that the spectrum of the chorus elements lacks the lower frequencies in the center of the source region, which can be explained on the basis of the BWO model of chorus generation. Titova et al. (2012) further investigated the capability of the BWO model to reproduce the 
nonlinear rising tone chorus elements that are observed in the WBD measurements. The $q$ parameter values contained within the BWO model that quantify the excess of the energetic electron flux above the absolute-instability threshold, calculated from Cluster observations (chorus waves, magnetic field, density) indicate a "large excess over the generation threshold $(q>3)$ resulting from numerical simulation of discrete elements with rising frequency, and are thus consistent with the simulations". In a more general theoretical investigation, Lakhina et al. (2010) studied the cyclotron resonance of the energetic electrons with the coherent chorus subelements and found that rapid pitch angle scattering may explain precipitation of $\sim 10-100 \mathrm{keV}$ electrons and the production of ionospheric microbursts of $0.1-0.5 \mathrm{~s}$ in bremsstrahlung $\mathrm{x}-$ rays formed by $\sim 10-100 \mathrm{keV}$ precipitating electrons. This association of chorus wave/electron interactions with microbursts was a new result.

Banded Chorus. Observations near the magnetic equatorial plane in the region where chorus is generated show that chorus often appears in two distinct frequency bands, one below and one above $f_{c e} / 2$. This is banded chorus and its existence has been known since 1969 but until recently there has been no satisfactory explanation of the phenomenon. Using WBD, Bell et al. (2009) showed that banded chorus can be explained if chorus is excited in ducts of either enhanced or depleted cold plasma. The result was later verified by Haque et al. (2011, 2012). The upper-band chorus is excited within depleted ducts and lower-band chorus is excited within enhanced ducts. Since the enhanced duct cannot guide whistler mode waves when $f=f_{c e} / 2$, there will be a small gap between the upper and lower chorus bands. This explanation also provides a mechanism for producing periodic chorus. The consequences of this explanation may have a far reaching effect upon our understanding of the conditions under which banded-chorus is excited and the locations where this excitation takes place. One of these is that different groups of energetic electrons may be involved in the excitation of the two chorus bands, even though the two bands are generally separated in frequency by less than $500 \mathrm{~Hz}$. A second consequence would be the limitation of the banded-chorus sources to a finite number of locations within the magnetosphere, i.e., the maxima and minima density as a function of $L$-shell. This limitation could affect the number of $10-100 \mathrm{keV}$ electrons that can be accelerated to $\mathrm{MeV}$ energies by banded-chorus waves at any given time.

Santolik et al. (2010) studied wave-particle interactions in the equatorial region of two-banded whistler-mode emissions observed by WBD. The data showed that the emissions were in the form of random hiss with only occasional discrete chorus wave packets and that the wave propagation properties were very similar to previously analyzed cases of whistler-mode hiss. They performed a linear stability analysis based on the locally-measured (by PEACE) electron phase space densities and found two unstable electron populations: The first consisted of energy-dispersed and highly anisotropic injected electrons at energies of a few hundreds of $\mathrm{eV}$ to a few $\mathrm{keV}$, with the perpendicular temperature more than 10 times higher than the parallel temperature. Another unstable population was formed by trapped electrons at energies above 10 $\mathrm{keV}$. They showed that the injected electrons at lower energies could be responsible for a part of the waves that propagate obliquely at frequencies above $f_{c e} / 2$ and suggested that a nonlinear generation mechanism might be necessary to explain the waves below $f_{c e} / 2$.

Schriver et al. (2010) used the same Cluster event and data as part of a theoretical study of the wave generation. Their theoretical results showed that the anisotropic electron distribution could linearly excite obliquely propagating whistler-mode chorus waves in the upper frequency band $\left(>f_{c e} / 2\right)$. They also showed that the lower 
frequency wave modes below $f_{c e} / 2$ were nonlinearly excited from the emissions above $f_{c e} / 2$ through wave-wave coupling. The instability could be saturated primarily by a decrease in the temperature anisotropy of the mid-energy electrons, but also by heating of the cold electron population.

Fine structure. The fine structure of the storm-time chorus was examined in detail in Santolik et al. (2003) who found that the chorus wave packets, appearing as rising discrete elements on the power spectrograms, have an internal fine structure consisting of separate subpackets of variable lengths up to $40 \mathrm{~ms}$. These subpackets seem to appear in the waveform in a random way without any clear periodicity. They also found that at the start of the majority of these subpackets there is an exponential growth phase which could be consistent with a linear instability or with the linear growth of the triggered emissions. Finally, they found that simultaneous observations on the four Cluster spacecraft showed that even if chorus elements are well correlated on the power spectrograms at time scales of $0.1 \mathrm{~s}$, their internal subpacket structure was different on the different spacecraft. This either means that the fine structure has a shorter characteristic dimension than a few tens of kilometers in the plane perpendicular to the ambient magnetic field or else that the fine structure changes along the field line at spatial scales of hundreds of kilometers.

Most of the significant advances (such as in the source region as well as the nature of the banded and fine structured emissions) since the early days of chorus research have come through Cluster multi-spacecraft operations. These advances concern primarily the nature of the waves within the chorus source region and their propagation away from this region. One surprising finding with regard to the latter is that there can be significant frequency differences between nearly identical discrete chorus wave packets that were observed simultaneously by different Cluster spacecraft. Some possible explanations have been put forth to explain this difference, however, further experiments with high time resolution and from multiple observing locations will be necessary to determine which, if any, of these explanations are viable.

\subsubsection{Electrostatic solitary waves.}

ESWs usually indicate the presence of potential or density structures and are related to both magnetic reconnection physics and particle acceleration. ESWs have been observed in space data since 1982 (Temerin et al. 1982) and are identified in electric field waveform data as pulses that are single-peaked, double-peaked (one positive and one negative peak of nearly equal amplitudes), or triple-peaked (two positive peaks with an intervening negative peak, or vice versa). The ESW pulse time durations are typically $\sim 10$ 's $\mu$ s up to several milliseconds. The amplitudes are anywhere from less than $100 \mu \mathrm{V} \mathrm{m}^{-1}$ in the Earth's foreshock region up to $2.5 \mathrm{~V} \mathrm{~m}^{-1}$ in the auroral acceleration region (see Lakhina et al. 2009). Spectral analysis indicates that the emissions are broadband. Thus, it is believed that the spectral wave observations made in space prior to the 1990s that were reported as Broadband Electrostatic Noise (BEN) probably indicated the presence of ESWs (see e.g., Temerin et al. 1982). ESWs (and BEN) appear to be ubiquitous in regions of mixing plasmas, such as boundary layers, and in other turbulent and dynamic plasmas. They are found in abundance in the auroral acceleration and magnetic reconnection diffusion regions, at the magnetopause, polar cap and plasmapause boundary layers, in the magnetosheath, in the solar wind, and at the bow shock. Lakhina et al. (2011) have recently provided a summary of papers published that discussed these observations, but there was no discussion of the results. 
ESWs observed in space are generally thought to result from nonlinear processes including ion and electron beam instabilities, two stream instabilities, and electron acoustic instabilities. The ESWs associated with electron (ion) beams have positive (negative) potentials and propagate at speeds on the order of the electron thermal velocity (ion beam or ion acoustic speed). Positive potential ESWs are usually interpreted in terms of the Bernstein-Greene-Kruskal (BGK) modes, i.e., phase space holes. They are also thought to result from the nonlinear evolution of the two stream instability and are a signature of electron-acoustic solitary waves. Lakhina et al. (2011) and Ghosh et al. (2008) reference publications that discuss the generation of ESWs.

A survey of where ESWs have been observed by the WBD instrument in its polar orbit $\left(\sim 4 \times 19 \mathrm{R}_{E}\right)$ is shown in Fig. 3 of Pickett et al. (2004a). The ESWs were found in all of the expected regions. The Cluster survey was the first time that the entire magnetosphere and the region just outside the bow shock were covered by a single mission. The survey highlighted a tendency of the electric field amplitudes of the ESWs to increase as the strength of the magnetic field increased over a range of 5-500 nT. This same trend, although not quite as strong, seems to be present at Saturn as well (Williams et al. 2006). An explanation, provided by Pickett et al. (2004a) is related to the structures being Bernstein-Greene-Kruskal (BGK) mode solitary waves (Chen and Parks 2002a,b). ESWs produced in this mode have widths and potential amplitudes that are constrained by inequalities, unlike fluid solitons such as $\mathrm{KdV}$ solitons (where there is only one allowed width for any given amplitude and the width increases with decreasing amplitude).

Trines et al. (2007) used Cluster EFW data obtained at the magnetopause to identify the spontaneous emergence of electrostatic solitary structures from broadband AC turbulence at the magnetopause boundary layer. The unique capabilities of Cluster enables their identification as separate entities. Numerical simulations provided an interpretation of these structures in terms of a nonlinear phenomenon generated during the interaction of short-scale drift wave turbulence and zonal flows. A Cluster study of ESWs upstream of the Earth's quasi-parallel bow shock (Behlke et al. 2004) showed for the first time that they can be embedded within short large-amplitude magnetic structures (SLAMS). These ESWs exhibit negative potential structures that usually are associated with ion beams and processes. However, none of the theories commonly invoked address the fact that these are negative potential structures that are moving at velocities above the ion thermal speed in a weakly magnetized plasma. Pickett et al. (2011) discussed the modulation of ESWs by PC1 waves as obtained by WBD near the dayside magnetopause on the Earth side. The ESWs occurred in bursts at the PC 1 wave frequency of $1.2-1.7 \mathrm{~Hz}$. Pickett et al. (2011) concluded that the component of the electric field parallel to the magnetic field of the PC1 wave acted as an induced electric field that accelerated electrons and ions along the local magnetic field as the PC1 wave propagated. This in turn set up the necessary conditions for the initiation of a Buneman-type instability that generated ESWS that were then modulated in bursts at the PC1 frequency.

Pickett et al. (2005) also looked at Cluster WBD waveforms obtained in the near-Earth magnetosheath and concluded that the ESWs were most likely locally generated by the two stream instability, but did not rule out other possible generation mechanisms, e.g., the lower-hybrid Buneman instability with an electron beam, the electron-acoustic mode, or spontaneous generation out of the general level of magnetosheath turbulence. Lakhina et al. (2009) carried out a theoretical study showing that these very same magnetosheath ESWs (Pickett et al. 2005) could arise from the electron acoustic mode, whereas Umeda et al. (2012) used a Vlasov simulation 


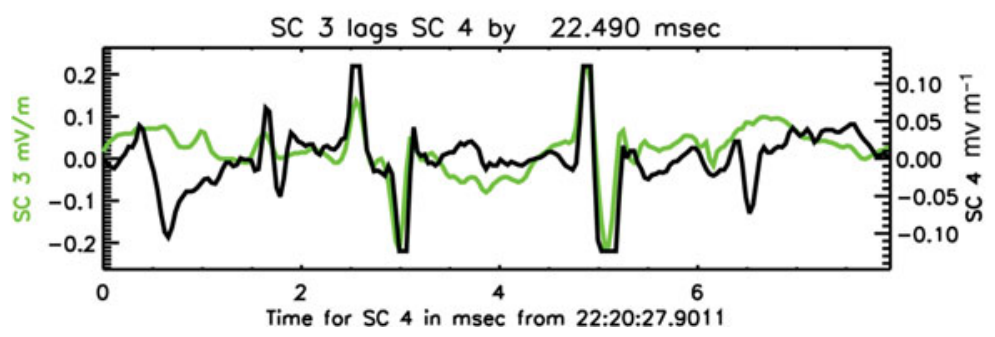

FIGURE 13. An example of ESW propagation from $\mathrm{C} 4$ to $\mathrm{C} 3$, where the $\mathrm{C} 3$ waveforms have been brought forward in time by the detected lag $(22.49 \mathrm{~ms})$ and overlaid on the $\mathrm{C} 4$ waveform (adapted from Fig. 5 of Pickett et al. 2008).

to show that they could only be generated through a bump-on-tail instability. This particular event was chosen by all three investigations because a special mutual impedance test had been run during the event, thus providing more information on the electron populations that were present than could normally be obtained in this region

Unfortunately, it is not usually possible to determine with certainty what mode any ESW originates in since the required particle distributions typically cannot be obtained with the time resolution required to determine with certainty whether the ESWs are associated with an ion or electron beam, a streaming instability, or an ion acoustic instability. In addition, since ESWs propagate, it is possible that the ESWs are being observed far from the generation region. Numerous spacecraft observations have shown ESWs propagating over just tens of meters (the antenna length), which is not sufficient to determine whether the ESWs are propagating small distances within the generation region or have come from large distances. However, some Cluster observations have found propagation over tens of kilometers (Pickett et al. 2004 b) by observing the same ESWs propagating from one spacecraft to another along auroral field lines outside the acceleration region and in the magnetopause boundary layer. Figure 13 is an example from the magnetopause boundary layer on the magnetosheath side showing propagation of a few ESWs from C4 to C3 using cross spacecraft correlation analysis to evaluate the waveforms. The lag in observation of the ESWs by C3 was found to be about $22 \mathrm{~ms}$, giving ESW velocity of $1334 \mathrm{~km} \mathrm{~s}^{-1}$ away from the Earth. The ESWs were thus found to be stable over a few tens of $\mathrm{ms}$, as opposed to the several tens or few hundreds of $\mu$ s for propagation observed between antennas on one spacecraft. This aspect of stability needs to be considered when developing theories to explain how ESWs interact with waves and particles.

Theoretical studies of ESWs point out that they probably evolve over time and sometimes interact with other ESWs. The time scales over which this happens depends on the specific environment in which the ESWs are found. However, the Cluster ESW propagation studies prove that ESWs are not intrinsically unstable, which helps to bound the problem when considering how they are generated. This was an important issue in the study by Mutel et al. (2006), which concluded that ion solitary waves passing through the AKR generation region affected the $\mathrm{CMI}$, leading to the striations discussed above.

Although all generation mechanisms mentioned above have been shown through theoretical models and simulations to reproduce the observations, there is still a controversy as to which mechanism dominates, or even if all of them are operating, albeit in different regions. In an effort to clarify this issue, laboratory experiments were 
carried out in the LArge Plasma Device (LAPD) at the University of California-Los Angeles. For a discussion of those experiments and the complementary simulations, the reader is referred to Lefebvre et al. (2011).

There is still a great deal of uncertainty as to which type of nonlinear instability leads to the generation of ESWs. To address this, future studies will need to concentrate on carrying out more laboratory measurements, obtaining higher time resolution particle measurements in space, and identifying the signature of local generation so as to determine if the ESWs are locally generated.

\subsubsection{ESWs and reconnection.}

A study by Cattell et al. (2005) using Cluster EFW waveform data in the magnetotail showed the presence of large-amplitude (up to $\sim 50 \mathrm{mV} \mathrm{m}^{-1}$ ) solitary waves. These solitary waves were identified as electron holes and were observed by two Cluster satellites during several plasmasheet encounters that were coincident with passage of a magnetotail reconnection $X$-line. Narrow electron beams with broad pitch angle distributions were present during the intervals when the electron holes were observed. As stated by Cattell et al. (2005), "The electron holes were seen near the outer edge of the plasma sheet within and on the edge of a density cavity at distances on the order of a few ion inertial lengths from the center of the current sheet". The association of the electron holes with the density cavity and electron beams, as well as with their locations along the separatrices, are consistent with PIC simulations (Cattell et al. 2005 ) that reproduced the Cluster data but only when a small guide field was included.

Later studies by Deng et al. (2006) and Li et al. (2010) using WBD showed that ESWs were observed in the vicinity of a magnetic null near a magnetotail reconnection region - identified in the near-earth tail when the current sheet became dramatically thin during a substorm. The amplitudes of the ESWs in the vicinity of the $X$-line range from $0.1-5 \mathrm{mV} \mathrm{m}^{-1}$, with larger amplitudes nearer the magnetic null points. Using a two-dimensional model for the reconnection diffusion region, they showed that ESWs can be generated in the diffusion region, and will then evolve along the magnetic field both in the outflow region and in the separatrix. Viberg et al. (2013) mapped the high frequency waves in the reconnection diffusion region using multiple Cluster satellites and found that ESWs were located nearest to the current sheet, i.e., furthest into the separatrix region, which has a stratified spatial structure. These ESWs were associated with counterstreaming electron populations. Langmuir waves were found in the outer part (closest to the inflow), while electron cyclotron waves are observed in different parts of the separatrix region.

Viberg et al. (2013) also reported for the first time that there were three main types of the high frequency emissions in the diffusion region: Langmuir waves, ESWs, and electron cyclotron waves. To study the relation of the different waveforms to electron distributions, the waveforms were compared with electron distributions measured at $125 \mathrm{~ms}$ cadence (one energy sweep of the PEACE electron detector) as the waveforms were changing on timescales of $\sim$ seconds. The three spacecraft spent several minutes in the diffusion region. Little or no activity was found in the inflow and outflow regions, and most of the wave activity was localized to the separatrix regions, which were crossed multiple times, revealing a spatially stratified structure.

Closest to the inflow, Langmuir waves were observed that were generated by suprathermal low density electron beams that were propagating away from the $X$-line. Thus, the authors concluded that the observation of the first Langmuir waves when the spacecraft were entering the diffusion region from 'the inflow was a signature of the separatrix (electron edge)'. In the inner part of the separatrix region, mostly 


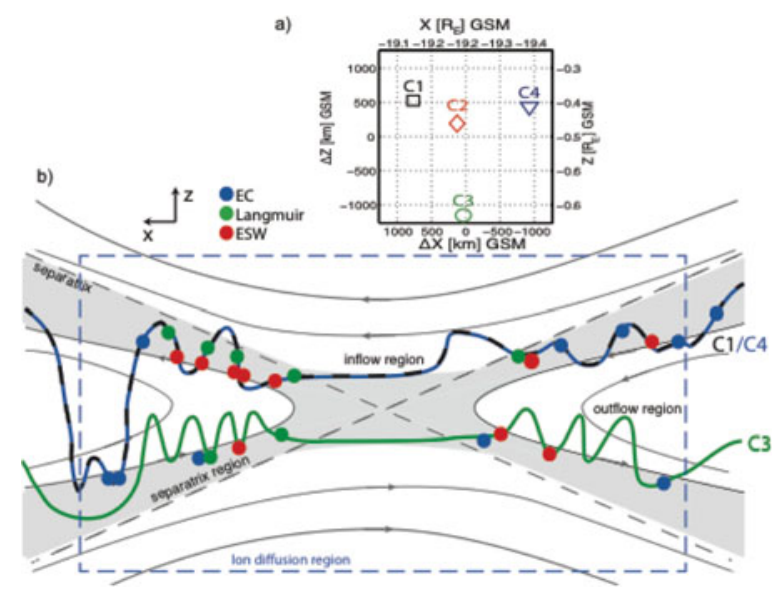

FIGURE 14. Sketch of the reconnection event, with (a) the positions of the Cluster spacecraft, and (b), a sketch of the diffusion region with the approximate paths of $\mathrm{C} 1, \mathrm{C} 3$, and $\mathrm{C} 4$. Wave observations are indicated by the colored solid circles and the different regions within the diffusion region (blue box) are labeled (from Viberg et al. 2013).

electrostatic waves were observed together with electron distributions that showed counter-streaming electron populations (low-energy towards the $X$-line, high-energy away from the $X$-line). Electron cyclotron waves "observed in different parts of the separatrix region" have the shortest timescales of the three observed wave types (down to several tens of milliseconds or several tens of electron gyroperiods). This short time scale possibly reflects fast relaxation of perpendicular electron anisotropies created in the diffusion region. A distinct boundary was seen in waves between the separatrix region and the central part of the exhaust, where no waves are observed. The investigation provided precise mapping of the kinetic boundaries. More measurements of this type will be made in this region and in the magnetopause as Cluster's orbit becomes more favorable for sampling regions where reconnection is likely to occur. Figure 14 from Viberg et al. (2013) illustrates a representative event, showing positions of the Cluster spacecraft in a reconnection diffusion region with the paths determined by the wave signatures detected by, C1, C3 and C4 as colored lines (black, green, red, respectively). Different wave modes (colored circles) are observed in different parts within the diffusion region (blue box).

\subsubsection{Magnetosonic solitons.}

The first experimental evidence for slow magnetosonic solitons in a natural unbounded collisionless plasma was provided by the four Cluster spacecraft while at the dusk flank of the magnetosphere (Stasiewicz et al. 2003). These solitons had characteristic magnetic field depressions (up to $85 \%$ ) accompanied by an enhancement in plasma density and temperature by a factor of two. The solitons were found to propagate at $250 \mathrm{~km} \mathrm{~s}^{-1}$ with respect to the satellites and have perpendicular sizes of 1000-2000 km (i.e., few $d_{i}$ ). The multipoint measurements made it possible to provide all of the relevant parameters of the solitons and their environment for the first time.

Stasiewicz (2004) applied a model of nonlinear waves in anisotropic plasmas, including electron inertial effects, to reinterpret mirror modes observed in the magnetosheath as trains of slow magnetosonic solitons. He obtained exact nonlinear magnetosonic wave-train solutions to the Hall-MHD equations with anisotropic ion pressure. The solutions had properties previously attributed to mirror-mode structures. 
Those solutions were compared with multipoint Cluster measurements taken in the magnetosheath. The computed properties of the nonlinear waves (amplitudes, spatial scales, periodicity, and propagation velocities) were consistent with the Cluster data.

The coupling of ion solitary waves to whistler mode waves was studied by Tenerani et al. (2012) who modeled a low frequency solitary wave observed by Cluster that was characterized by a magnetic dip and a density hump, as a nonlinear coherent wave, a solitary wave or a slow magnetosonic soliton, propagating at subsonic speed quasi-perpendicular to the magnetic field. The scale of the solitary wave was of the order of the ion-scale, suggesting that the low frequency wave was a solitary kinetic Alfvén wave. The soliton trapped and advected the high frequency waves over many ion times, which is much longer than their characteristic electronic time scale.

\subsection{Bow shock studies}

The Earth's bow shock was discovered in 1964 (Ness et al. 1964) and subsequently, bow shocks have been observed at all of the visited planets (Treumann and Jaroschek 2008), at comet Halley (Coates et al. 1997), and, most recently, at the termination shock that separates the solar system from interstellar space (see for example, Stone et al. 2005). Soon after the Earth's bow shock was discovered, Kaufmann (1967) noted that some shock transitions from upstream to downstream were sharp and well defined, while others were noisy and accompanied by large magnetic fluctuations. Many years of shock studies have since shown that an important parameter that organizes the bow shock is the angle between the IMF and the normal to the boundary of the shock, $\theta_{B N}$ (Greenstadt et al. 1970). The bow shock is classified as either quasi-perpendicular $\left(\theta_{B N}>45^{\circ}\right)$ or quasi-parallel $\left.\theta_{B N}<45^{\circ}\right)$. On average, the quasi-perpendicular shocks favor the dusk side and quasi-parallel geometry the dawn side. Quasi-perpendicular shocks are relatively stable while the quasi-parallel shocks are usually accompanied by waves and turbulence. Theories predict that heating in the quasi-parallel region is more efficient because of the presence of waves and turbulences. (See for example, Kennel et al. 1985; Wilkinson 2003; Chapman et al. 2005; Scholer et al. 2005).

A shock is subcritical when the Alfvén Mach number is $<2-3$ and super-critical when the Mach number is $>2-3$. Earth' bow shock is more often than not supercritical and only rarely sub-critical (Thomsen 1988). The magnetic profile of supercritical shocks includes a 'foot' produced by a fraction of reflected ions, a ramp and 'overshoot' due to gyrating particles that is often followed by an 'undershoot'. These profiles have scale lengths of the order of an ion Larmor radius. The subcritical shocks do not have a foot or overshoot and some theories predict that the solar wind can be transmitted across them more easily. The foot and overshoot have been seen in hybrid simulations (Leroy et al. 1981).

Many questions about the SW interaction with the bow shock and mechanisms that cause dissipation and heating remain poorly understood (Krall 1997). At the present time, there are at least two possible ways to interpret the source of high temperature $(\sim$ few $\mathrm{keV})$ plasmas observed in the magnetosheath. One attributes the hot magnetosheath plasma to heating of the solar wind by instabilities at the shock (Auer et al. 1971; Wu and Yoon 1990; Gedalin 1997; Ellacott and Wilkinson 2003). There are many possible instabilities, but none has been specifically identified.

Another attributes the high temperature to reflected solar wind particles. The reflected population in the upstream is accelerated by the solar wind electric field and when it subsequently flows back across the bow shock with the solar wind, it occupies a different region of the phase space. The resulting dispersion of phase 
space density increases the volume in the velocity space, effectively increasing the kinetic temperature (Paschmann 1983; Gosling and Robson 1985). The ion cyclotron waves observed in the magnetosheath along with the hotter distributions suggests that waves are excited along the way, interacting with the particles to form bi-Maxwellian distributions (Sckopke 1995).

Recently, Cluster observations of quasi-parallel and quasi-perpendicular shocks in conjunction with PIC simulations has further contributed to our understanding of bow shock physics (Burgess 1989; Lembège and Savoini 2002; Scholer et al. 2003; Wilkinson 2003; Burgess et al. 2005; Burgess 2006; Lucek et al. 2008). For example, the motions and thicknesses of the quasi-perpendicular shock ramp region can now be determined accurately. The PIC simulations have also predicted that the bow shock can undergo reformation (see Horbury et al. 2002; Chapman et al. 2005).

New information obtained on upstream phenomena includes low frequency magnetic fluctuations observed in the quasi-parallel shock region (for example, SLAMS) and high frequency whistler mode wave packets that convect with the solar wind across the bow shock into the magnetosheath and must be treated as an ensemble (Burgess 1989). However, many other details of the solar wind interaction with the bow shock remain unknown.

Here, we describe a study that focussed on how the solar wind dissipates across the bow shock. The behavior of ions is emphasized because they carry the bulk of the solar wind energy and momentum. Moreover, the Cluster separation strategy is adapted to ion dynamics (MMS will focus on electron dynamics). The questions we address are: (1) How does the solar wind interact with the bow shock and is there any heating of the directly transmitted ions as they cross the shock into the magnetosheath? (2) What upstream solar wind and bow shock conditions allow the solar wind particles to be directly transmitted into the magnetosheath? (3) What waves are observed along the way to the magnetopause leading to Maxwellian or bi-Maxwellian distributions with $T_{\perp}>T_{\|}$?

\subsubsection{Entropy conservation across the Earth's bow shock.}

There are still facets of collisionless shocks that are little understood. These issues basically involve how incoming plasma thermalizes and how entropy is generated. Earth's shock is thought to be an excellent example of a high Mach number shock. The shock itself is, to all intents and purposes, collisionless - its width is significantly smaller than the collision mean free path at 1 AU. This fact raises the question as to what physical mechanisms exist that can dissipate energy and generate entropy. Recent analysis of Cluster data has demonstrated that the width of the Earth's bow shock is much less than an ion Larmor radius (Schwartz et al. 2011), and is actually on the order of a few electron inertial lengths $(\sim 20 \mathrm{~km})$.

Parks et al. (2012), using data from Cluster, looked at how entropy behaves across the Earth's bow shock. Classical thermodynamics defines the entropy of a system as the sum of the heat $(Q)$ divided by the temperature $(T)$. The extended classical definition of Gibb's entropy is $S=-k_{B} H$, where $H=\int f \ln f d^{3} v, f$ is the one-particle velocity distribution function, $k_{B}$ is Boltzmann's constant and the integration is performed over all velocities. The time rate of change of $H$ is given by $d H / d t=\int(1+\ln f) \partial f / \partial t d^{3} \mathbf{v} \leqslant 0$ where the equality holds only if the probability distribution $f$ is Maxwellian. $H$ is always negative and given that a system can be in many different configurations, $H$ will decrease to a minimum as $f$ evolves to the most probable distribution, corresponding to a state of maximum entropy. 


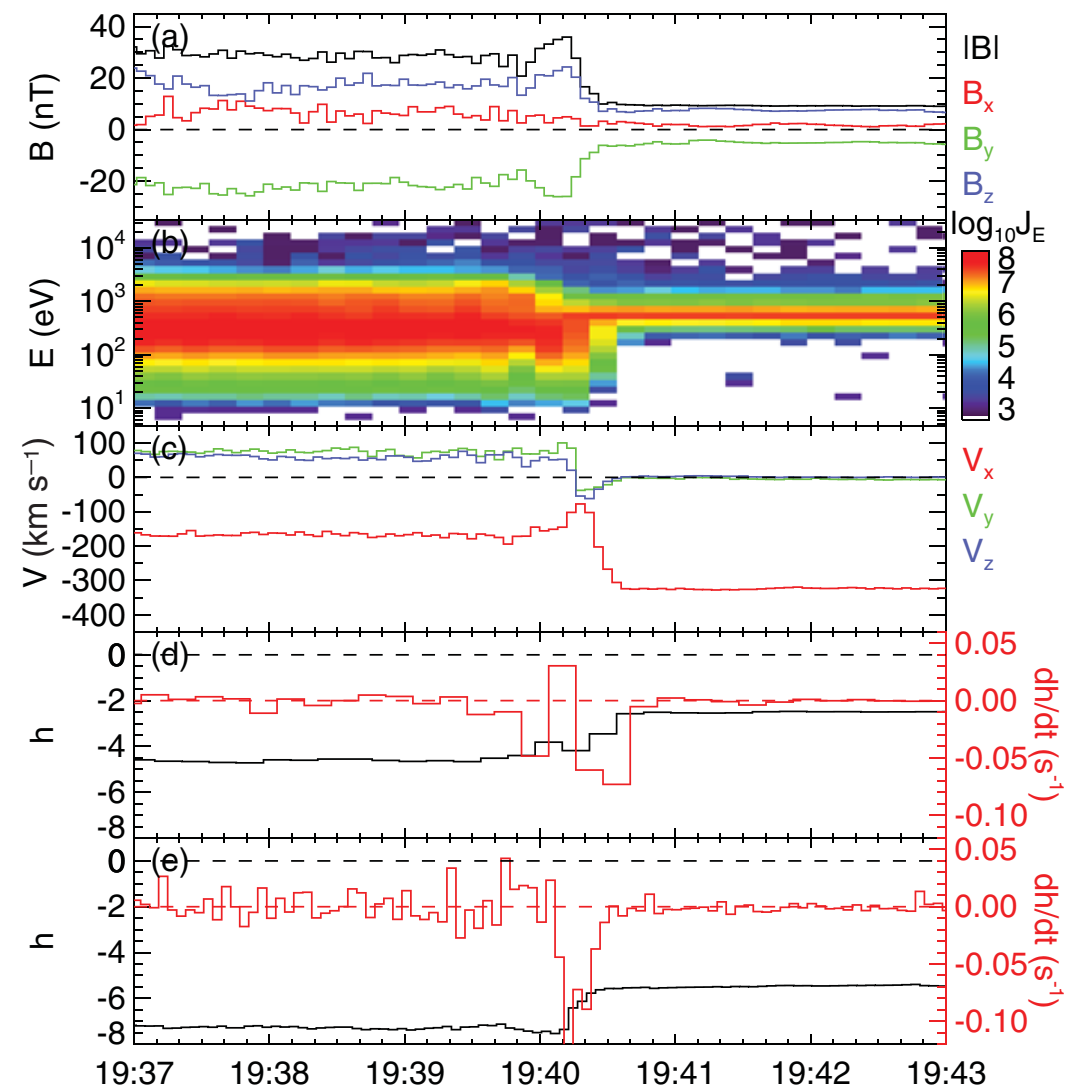

FIGURE 15. Bow shock crossing on 1 February, 2002. Ion data come from CIS and are 3 spin (12 s) averages. 3D distributions of electrons from PEACE, measured every spin, from C2 (no $3 \mathrm{D}$ data on $\mathrm{C} 1$ ). $\mathrm{C} 2$ was $\sim 600 \mathrm{~km}$ from $\mathrm{C} 1$ (the bow shock crossing time has been shifted to coincide with C1). From top to bottom: (a) $B$ field and components in the geocentric solar ecliptic coordinate (GSE) system, (b) energy spectrogram of ions, (c) mean velocities, (d) $h$ (black) and $d h / d t$ (red) of ions, (e) $h$ (black) and $d h / d t$ (red) of electrons (Parks et al. 2012).

The plasma experiments on Cluster and Double Star routinely measure 3D particle distributions $f(r, v, t)$ (Johnstone et al. 1997; Rème et al. 1997). The spacecraft motion for the most part is negligible compared with the motion of the solar wind across the shock. Thus, observed 'temporal' variations can be assumed to be the result of spatial structures passing over the spacecraft. As such, the measurements along the spacecraft track can then be interpreted as a history of the plasma volume that traveled the same track. Because the particle instruments acquire $f(\mathbf{v})$ only at fixed locations, and not throughout the flux tube, a normalized $H$ function was used in the analysis; $h=\sum p_{i} \ln p_{i}$, where $p_{i}=f_{i} \Delta^{3} v_{i} / N, N$ is particle number density and $i$ indexes the sampled phase space volume elements. This calculated $h$ is proportional to entropy per particle (entropy density) at the spacecraft. The normalized $d h / d t=[h(t)-h(t-\Delta t)] / \Delta t$ is calculated from successive measurements, where $\Delta t$ is the spacecraft spin period.

Figure 15 shows an example of how $h$ and $d h / d t$ change across the bow shock. The observations were made on 1 February, 2002 by $\mathrm{C} 1$. C1, initially in the magnetosheath, was outbound and crossed the bow shock at 1940:24 UT, identified from magnetic field data (panel a). The solar wind in the energy flux spectrogram plot appears as a narrow red line centered around $\sim 600 \mathrm{eV}$ (panel b, after $1940 \mathrm{UT}$ ). The solar 
wind flow speed was $V_{x} \sim-320 \mathrm{~km} \mathrm{~s}^{-1}$ which slowed down to $\sim 75 \mathrm{~km} \mathrm{~s}^{-1}$ and deviated in the $y$ - and $z$-directions just before crossing the shock (panel c). The flow speed settled to about $-150 \mathrm{~km} \mathrm{~s}^{-1}$ in the magnetosheath. The plasma in the downstream magnetosheath covers a broad energy range, from $\sim 10 \mathrm{eV}$ to several $\mathrm{keV}$ (panel b, before $1940 \mathrm{UT}$ ). The magnetic field (Balogh et al. 2001) on the four Cluster spacecraft show the boundary normal of this shock is $n=(0.7,0.7,0.3)$ and the angle between the normal and the $B$-field is $\theta_{B N} \sim 82^{\circ}-88^{\circ}$. This shock was moving along its normal with a speed $\sim 9 \mathrm{~km} \mathrm{~s}^{-1}$ and the Alfvén Mach number was $M_{A}=\left(V / V_{A}\right) \sim 3.0-3.5$, classifying it as supercritical perpendicular shock.

The black traces in panels (d) and (e) in the figure show $h$ for ions and electrons respectively. The electron data come from $\mathrm{C} 2$, which was separated from $\mathrm{C} 1$ by $\sim 1000$ $\mathrm{km}$. At this time, there were no electron data from $\mathrm{C} 1$. The plot shows that $h$ in the solar wind was -2.4 for ions and -5.5 for electrons. These values decrease across the magnetic ramp to -4.5 and -7.2 in the magnetosheath. Note that the transition of electrons occurs more rapidly than does that of the ions. The corresponding values of $S=-k_{B} H$ in the solar wind are $3.3 \times 10^{-16}$ ergs $\mathrm{K}^{-1}$ and $7.6 \times 10^{-16}$ ergs $\mathrm{K}^{-1}$ and in the magnetosheath $\sim 6.2 \times 10^{-16}$ ergs $\mathrm{K}^{-1}$ and $9.9 \times 10^{-16}$ ergs $\mathrm{K}^{-1}$. The increases of entropy across the shock are $\Delta S$ of $\sim 2.9 \times 10^{-16}$ ergs $\mathrm{K}^{-1}$ for ions and $2.3 \times$ $10^{-16}$ ergs $\mathrm{K}^{-1}$ for electrons. The time variation of $d h / d t$ calculated across the shock shows that $d h / d t$ starts out around $\sim 0$ in the solar wind consistent with the solar wind distributions being nearly thermal (red, panels (e) and (f)). $\partial f / \partial t$ is estimated from $[f(\mathbf{r}, \mathbf{v}, t)-f(\mathbf{r}, \mathbf{v}, t-\Delta t)] / \Delta t$, where $\Delta t=4 \mathrm{~s}$, the spacecraft spin period. Note that Coulomb collisions cannot be responsible for variations of $f$.

The red traces in panels (d) and (e) show $d h / d t$ which turns negative in the magnetic ramp to $-0.07 \mathrm{~s}^{-1}$ for ions and $-0.13 \mathrm{~s}^{-1}$ for electrons. The corresponding rate of entropy change across the shock is thus $\sim 0.1 \times 10^{-16} \mathrm{ergs} \mathrm{K}^{-1} \mathrm{~s}^{-1}$ for ions and $\sim 0.18 \times$ $10^{-16}$ ergs $\mathrm{K}^{-1} \mathrm{~s}^{-1}$ for electrons. The departure of $d h / d t$ from 0 at the ramp indicates that the distributions are non-thermal and the plasma is not in equilibrium. Similar to the behavior of $h, d h / d t<0$ for ions covers a broader region, extending from upstream solar wind to the downstream magnetosheath, whereas $d h / d t<0$ for the electrons is approximately limited to the magnetic ramp region. The entropy change seen is intimately tied to mechanisms that produce the non-thermal distributions at the shock. These results, modeled with the Vlasov theory of how entropy flux should behave across the shock, show the agreement is quite good (Parks et al. 2012).

After some manipulation, the collisionless Vlasov equation $(\partial f / \partial t+\mathbf{v} \partial f / \partial \mathbf{r}+\mathbf{a}$. $\partial f / \partial \mathbf{v}=0)$ can be written, when integrated over velocity space, as:

$$
\partial(n s) / \partial t+\nabla \cdot \int\left(-k_{B} \mathbf{v} f \log f\right) d \mathbf{v}=0,
$$

where $n=\int f d \mathbf{v}$ is the number density and $n s=\int f \log f d \mathbf{v}$ is entropy flux. Equation (2.2) is the entropy conservation equation and the second term is the divergence of the entropy flux computed kinetically. Let $\mathbf{v}=\mathbf{U}+\mathbf{c}$, where $\mathbf{U}$ is the velocity moment so the change of the variable just shifts the velocity axis. Define $f^{\prime}(\mathbf{c})=f(\mathbf{U}+\mathbf{c})$ so that (2.2) becomes $\partial(n s) / \partial t+\nabla \cdot(\mathbf{U} n s)=k_{B} \nabla \cdot \int \mathbf{c} f^{\prime} \log f^{\prime} d \mathbf{c}=0$ for equilibrium processes. Assume now a steady state and 1D bow shock with $x$-direction normal to the shock, in which case $d\left(\mathbf{U}_{x} n s-F_{x}\right) / d x=0$, indicating that $\left(\mathbf{U}_{x} n s-F_{x}\right)$ is a constant. Hence $\left(U_{x 1} n_{1} s_{1}-F_{x 1}\right)=\left(U_{x 2} n_{2} s_{2}-F_{x 2}\right)$, and using mass conservation $U_{1} n_{1}=U_{2} n_{2}$, gives

$$
s_{2}-s_{1}=\left(F_{2 x} F_{1 x}\right) / U_{1} n_{1},
$$

where the sub-indices 1 and 2 are quantities measured in the upstream and downstream regions. $U_{1}$ is the flow in the normal direction, which is determined from the 


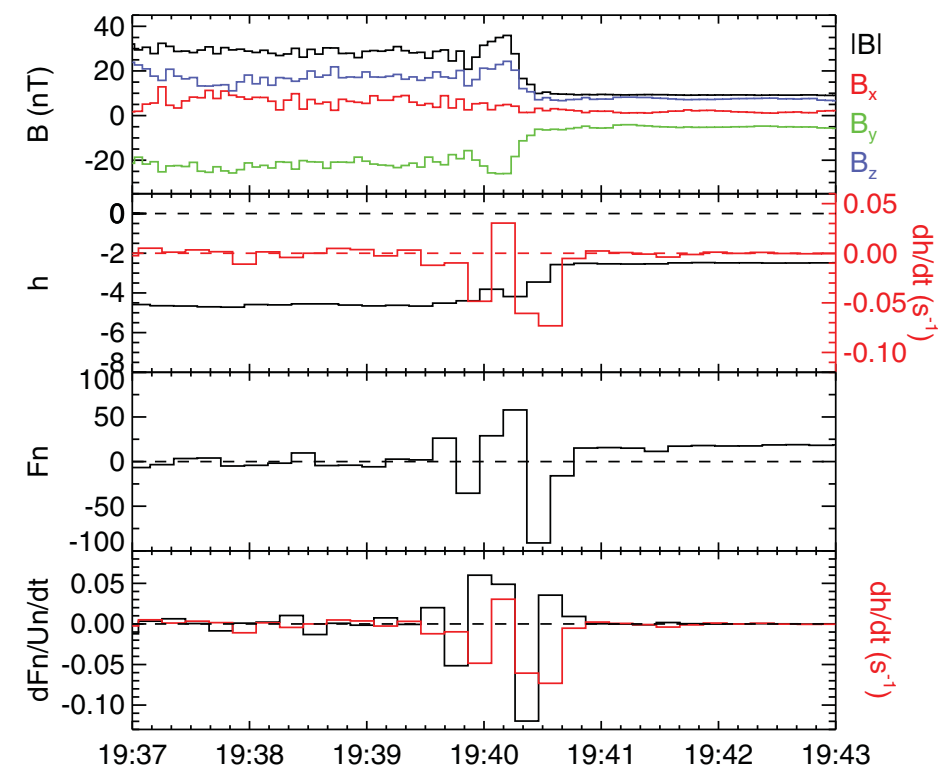

FIGURE 16. The top panel shows the magnetic field and components. Panel 2 shows $h$ and $d h / d t$. Panel 3 shows the kinetic function $F_{n}$. Plotted in panel 3 is the local change of the per particle entropy (black), (2.3), superposed on $d h / d t$ (red).

variance analysis. This equation shows that for processes that produce non-Maxwellian distribution functions, Vlasov theory predicts that the right side gives the amount of per particle entropy change.

The left side $\left(s_{2}-s_{1}\right)$ of $(2.3)$ has already been computed (Fig. 15). Figure 16 shows the new terms on the right of (2.3) for ions. The fact that the behaviors of $d h / d t$ (red) and $\left(F_{2} F_{1}\right) / U_{1} n_{1}$ (black) are 'similar' does not prove the validity of Vlasov theory, but rather indicates that the results generally support the simple Vlasov plasma model. Note that the per particle entropy is increasing across the (perpendicular) shock. Although Vlasov theory allows for generation of per particle entropy, the total entropy must remain constant because the Vlasov equation is symmetric in time and, therefore, is entropy conserving.

The above treatment has its limitations. The entropy equation used includes only the distribution function of charged particles, and as noted, hard collisions cannot be the source of the energy distribution in traversing the shock, but could be due to scattering by the large variety of complex waves that permeate the shock region (Gurnett 1985). These waves can have large amplitudes, short bursty durations, and be frequency dependent (with the frequencies of electrostatic waves $>f_{c e}$ and electromagnetic waves $<f_{c e}$ ). Furthermore, the electromagnetic emissions below the $f_{c i}$ are generally observed in a broader region of the shock area beginning in the foot region and extend into the magnetosheath while the narrower higher frequency electrostatic waves appear mostly in the magnetic ramp and may be important for heating particles in the magnetosheath.

\subsection{Solar wind plasma}

\subsubsection{The electron strahl.}

In many, if not most plasma environments, the fluid is far from being Maxwellian. Although such distributions may not be unstable, they will not relax to a single 
equilibrium Maxwellian in times of dynamic interest or importance. The solar wind is an example of such a plasma. In the solar wind, the electron (velocity) distribution functions (VDFs) are comprised of three and sometimes four separate components. About $95 \%$ of the solar wind electron density resides in the core, a thermal population generally existing below $10-15 \mathrm{eV}$ that is well represented by an isotropic Maxwellian distribution. The halo electrons are a non-thermal, slightly anisotropic population covering the energy range $\sim 12-100 \mathrm{eV}$. This component has been successfully modeled by both a Maxwellian slightly hotter than the core, and by a bi-Lorentzian kappa-like VDF (Feldman et al. 1975; Maksimovic et al. 1997; Nieves-Chinchilla and Viñas 2008). The third component of the solar wind is the strahl (German for beam), which is a field-aligned, beam-like, highly anisotropic population with energies between $0.05-2 \mathrm{keV}$. This population exists within a pitch-angle cone $\alpha<90^{\circ}$. The strahl was first described by Rosenbauer et al. $(1976,1977)$. Later studies showed the importance of the strahl in the overall anisotropy of the total electron VDF and for the heat-flux (Pilipp et al. 1987a,b,c; Salem et al. 2003). When present, the super-halo (Lin et al. 1997) constitutes a fourth component of the solar wind.

Together, the halo and strahl comprise the main source of the solar wind heat flux and are largely responsible for the overall electron temperature anisotropy. When the location of the measurement is magnetically connected to the Earth's bow shock, another population of electrons is often observed; a component that has either been reflected off the shock or has leaked back upstream from behind the shock. Those electrons move opposite to the strahl and in general cover a much wider energy and pitch-angle range. The presence or absence of this population is used to indicate when spacecraft are in the foreshock region or in the pristine solar wind.

Detailed measurements of the electron VDFs provide information about both interplanetary conditions and, to some extent, about the conditions in the solar corona. The morphology of the local electron VDF in the solar wind provides signatures that serve as a proxy for the (non-local) global structure of the magnetic and electric fields. For example, counter-streaming strahls have been observed on field-lines threading interplanetary coronal mass ejections (ICMEs), suggesting that the local field lines were connected to the Sun at both ends (Gosling et al. 1993, also see Gosling 1990).

\subsubsection{The electron velocity distribution function.}

The properties of the strahl have been explored by Viñas and Gurgiolo (2009) using a technique that separates strahl data from the core-halo electrons. Figure 17 shows an example of the technique for a typical electron VDF observed by PEACE on March 19, 2006. Each plot in the figure shows the velocity space density as a function of instrument elevation $(\theta)$ and azimuth $(\phi)$ for a single returned energy step in the plasma flow frame. The energy corresponding to each plot is shown to the right and left sides of the figure. The potential corrected energy is the lower number in blue. The top of each plot shows the maximum and minimum values of the VDF. The color scale for each plot runs linearly from the minimum to the maximum value. The circle $(\bullet)$ and triangle $(\boldsymbol{\Lambda})$ symbols in each plot are the projection of the head and tail, respectively, of the spin-averaged magnetic field vector. The populations of particles centered on $\pm 180^{\circ}$ at the lowest two energies of Fig. 17 are part of the solar wind core-halo population. The ring centered on the magnetic field head at $\sim 47.9 \mathrm{eV}$ are reflected/leaked particles that extend that extend in energy well above those shown. The strahl begins at $\sim 70.5 \mathrm{eV}$ where there is a shift in the local VDF away from $\pm 180^{\circ}$ towards the magnetic tail. The shift occurs at the energy where the strahl, which is field-aligned, begins to dominate the core-halo. 


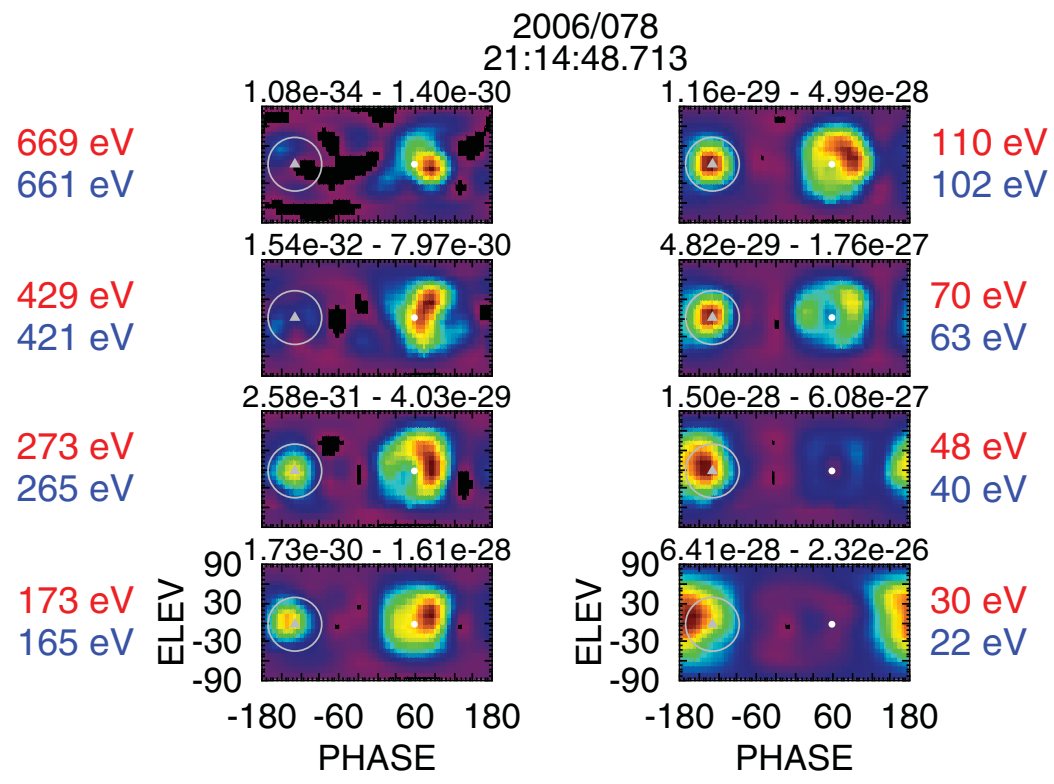

FIGURE 17. Set of $8 \phi-\theta$ showing the angular mapping for a subset of the 30 energy steps in a VDF. The energy steps shown are not contiguous. The phase is in GSE with the sun at $0^{\circ}$. (Adapted from Viñas and Gurgiolo 2009).

By defining a region of interest in the $\phi-\theta$ plots it is possible to isolate various populations. The circles in Fig. 17 delimit the strahl. On can estimate the fluid moments by setting all measurements outside the circle to 0 and then integrating over what is left. As an example, Fig. 18 shows plasma moments for both the strahl and core-halo populations for a time interval when the spacecraft were in fast wind $\left(>600 \mathrm{~km} \mathrm{~s}^{-1}\right)$ (January 10, 2004). The strahl density is restricted in range, lying between $0.05-0.1 \mathrm{~cm}^{-3}$.

Viñas et al. (2010) investigated the stability of the solar wind electron VDF in the presence of the strahl, parallel drifts, and the temperature anisotropy. The electron and magnetic field data (Fig. 18) together with CIS proton data were used to derive the parameters required in the dispersion analysis. Plotted are the ratio of plasma to cyclotron frequency, the core+halo and strahl densities normalized to total density, the parallel drift velocity of the strahl normalized to the core+halo parallel thermal velocity, the parallel $\bar{\beta}_{\| e}$ for the core+halo and strahl and the parallel $\bar{\beta}_{\| p}$ of protons. The parallel plasma $\bar{\beta}_{\| s}$ is representative of the parallel temperature and is defined as $\bar{\beta}_{\| s}=\left(8 \pi n_{\text {etot }} T_{\| s} / B^{2}\right)$ for each plasma component.

The dispersion analysis assumed a plasma composed of an isotropic core thalo, a field-aligned, anisotropic strahl, and a proton population in a homogeneous medium with a constant magnetic field. The calculation was carried out by numerically solving the dispersion relation (Gary 1993) for parallel propagating electromagnetic whistler waves. The details of the dispersion relation calculation can be found in Viñas et al. (2010). They concluded that whistler waves (with $60 \leqslant \omega_{r} / \Omega_{c p} \leqslant 120$ ) could be excited by a strahl temperature anisotropy of two. The peak in the growth rate decreases and the overall range narrows as the anisotropy decreases. The reverse occurs as the temperature anisotropy increases. The effect of the parallel drift velocity is opposite to that of the temperature anisotropy: the peak in the growth rate 


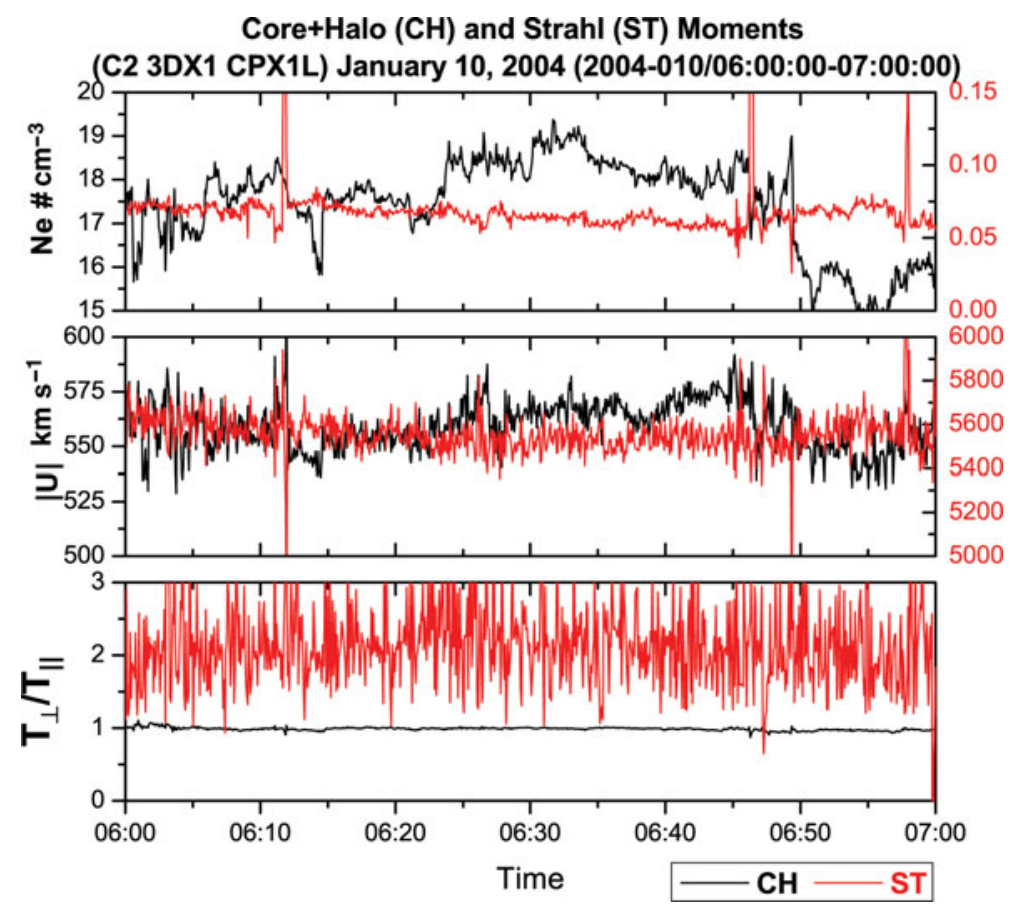

FIGURE 18. Computed density, velocity, and temperature anisotropy for the strahl (red) and core+halo (black) on January 10, 2004 when the spacecraft were in fast solar wind. The strahl density and velocity are plotted against the right $y$-axis (from Viñas et al. 2010).

decreases and the overall range narrows as the drift velocity increases. The opposite occurs when the drift decreases (because the temperature anisotropy is kept fixed at 2 ), and, as the drift velocity decreases, the effective number of particles that come into resonance with the waves increases. Of the two free energy sources, whistler wave excitation is the one more sensitive to the strahl temperature anisotropy.

While it is possible that a large temperature anisotropy $\left(T_{\perp} / T_{\|} \simeq 0.6-0.8\right) \mathrm{might}$ excite the whistler fire-hose instability, or that the free energy in the relative drift between the core+halo and an isotropic strahl might be sufficient to excite the whistler heat flux instability, analyses indicate that under nominal conditions neither instability is viable as they require a parallel electron $\bar{\beta}_{\| e}>4$, while the measured values are $1.5-2$.

Questions remain, however. For example, what creates and sustains the strahl temperature anisotropy, and what role does the strahl temperature anisotropy play in the stability of the full solar wind distribution? As discussed above, large temperature anisotropies are unstable to whistler waves (Gary 1993), which produce enough pitchangle scattering to regulate the full electron VDF. Rapid transitions of the strahl from an anisotropic to nearly isotropic distribution suggests that local effects must be important in scattering the strahl. One possibility is that the turbulent cascade to electron scales (see e.g., Bale et al. 2005; Sahraoui et al. 2009, and Sec. 2.7) may provide sufficient pitch-angle scattering to both drive the anisotropy and broaden the angular width of the strahl. Another possibility is the suggestion of Maksimovic et al. (2005) that the halo electrons are generated by pitch angle scattering of the strahl. In either case, it is clear that the strahl plays an important role in the regulation and evolution of solar wind electrons. 


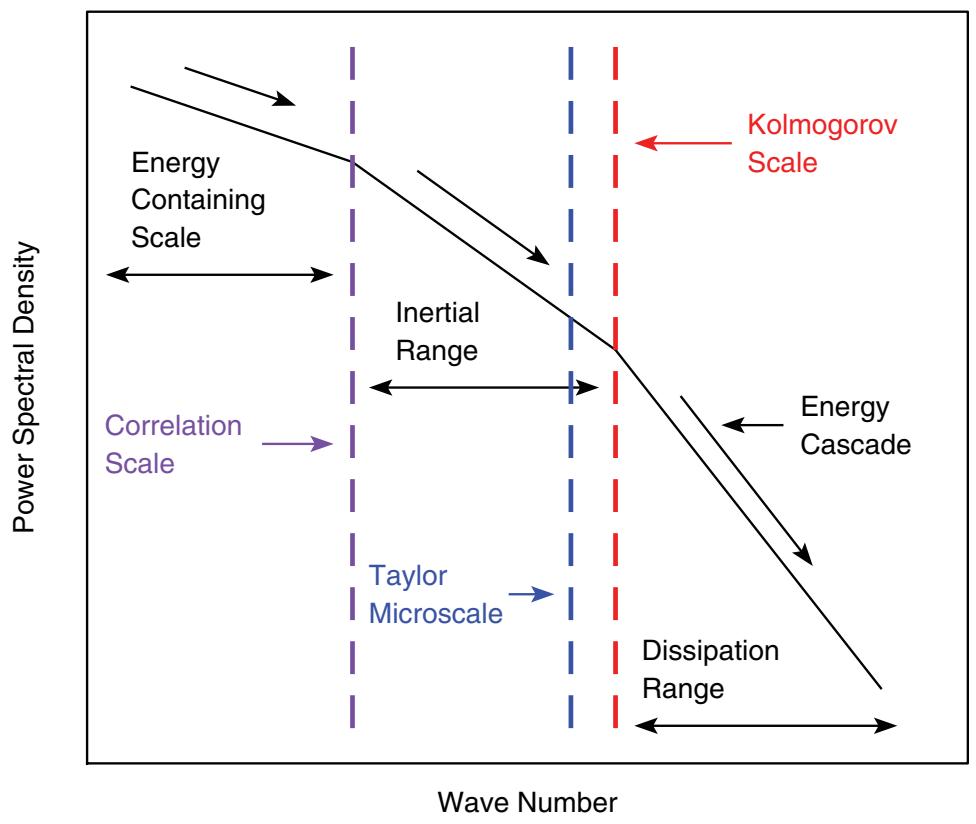

FigURE 19. Generic turbulence spectrum showing the typical ranges and scales.

\subsection{Turbulence}

Turbulence is an efficient means of transporting energy, momentum, and mass in a fluid system. A turbulence spectrum is generally thought of as being divided into three ranges (Fig. 19). Breaks in the spectra occur in the vicinity of the correlation and Kolmogorov scales. Most of the energy is in the 'energy-containing range' that comprises the lowest frequencies and the largest eddy structures. Eddies are generally nonlinearly unstable and the break in the spectrum at the correlation scale represents the size of the largest eddy in the system. The inertial range covers the central portion of the spectrum that contains eddy sizes between the largest and those that begin to be influenced by dissipation. The cascade in energy (eddy size) is dominated here by inertial or inviscid processes. Below the inertial range is the dissipative range where energy dissipates. The dissipation range is delimited by a change in spectral index at the small wave number end of the inertial range. Beyond this scale eddies are overdamped. For a viscous fluid, just above the Kolmogorov scale lies the third fundamental scale, the Taylor microscale $\left(\lambda_{\mathrm{T}}\right)$, discussed further below.

\subsubsection{The magnetofluid turbulence correlation scale.}

The correlation scale $\lambda_{C S}$, which is typically of the same order as the energycontaining scale, can be measured in the super-sonic and super-Alfvénic solar wind using classical methods based on the assumption of the validity of the Taylor frozen-in flow hypothesis (Taylor 1935). However, using simultaneous two-point measurements of turbulent fluctuations at a variety of spatial separations, one can relax the frozen-in flow approximation when determining the correlative scale. Matthaeus et al. (2005) used simultaneous two-point measurements from the Cluster, ACE, and Wind spacecraft to construct a correlation function for all solar wind conditions. They obtained a correlative scale value of $1.2 \times 10^{6} \mathrm{~km}$. Weygand et al. (2007, 2009 , 2011) expanded the Matthaeus et al. (2005) study and accumulated two-point spacecraft samples from 11 different solar wind spacecraft, including Cluster, in 
sufficient numbers to resolve the correlations into angular bins relative to the locally computed mean magnetic field for both the solar wind and the Earth's plasma sheet. The solar wind correlation scale they found varied from $1.0 \times 10^{6} \mathrm{~km}$ to $2.8 \times$ $10^{6} \mathrm{~km}$ and the plasma sheet correlation scale varied between 9000 and $16000 \mathrm{~km}$, the exact numbers for each case depended on the angular bin. Weygand et al. (2011) went on to demonstrate that there was a variation of the correlation scale with the angle relative to the mean magnetic field direction in both the slow and fast solar wind. In the slow solar wind, the ratio of the parallel (to the mean magnetic field) correlation scale to the perpendicular correlation scale was $2.55 \pm 0.76$ and became $0.71 \pm 0.29$ in the fast solar wind, which was similar to the previous results from Dasso et al. (2005) and Osman and Horbury (2007). Dasso et al. (2005), using a single spacecraft found a value of 1.2 for slow solar wind and 0.71 in fast solar wind while Osman and Horbury (2007) used multispacecraft time-lagged two-point correlation measurements from Cluster and found that in slow solar wind the ratio was $1.79 \pm 0.36$. A similar anisotropy of about $2.37 \pm 0.44$ was observed in the magnetic field fluctuations deduced from Cluster data in the Earth's plasmasheet during quiet geomagnetic conditions (Weygand et al. 2010). However, during active geomagnetic conditions the ratio of the parallel correlation scale to the perpendicular correlation scale becomes approximately 1 . Weygand et al. (2010) suggested that the variation of the correlation scale with geomagnetic activity could mean either that there was a change in the scale size of the turbulence driver or, alternatively, a change in the predominance of one over another type of turbulence driving. The fact that the correlation scale varied in both the solar wind and plasma sheet with respect to the mean magnetic field direction is important to studies of solar and galactic cosmic ray scattering. In both regimes, the perpendicular diffusion coefficient that describes particle scattering is directly proportional to the correlation scale (Ruffolo et al. 2004), while the parallel scattering rate depends on the anisotropy of the turbulence in the inertial range (Bieber et al. 1994).

\subsubsection{D spectra: $k$-filtering.}

Analyses of space plasma turbulence using data from single spacecraft suffer from a spatio-temporal ambiguity, viz., the difficulty of disentangling temporal and spatial variations. This issue is acute for magnetofluid turbulence in the solar wind where it is very difficult to deduce the three-dimensional properties of the turbulent fluctuations from single spacecraft data, notwithstanding the early efforts of Matthaeus et al. (1990), Bieber et al. (1996), Dasso et al. (2005). Using techniques such as $k$-filtering (or, the wave telescope) (Neubauer and Glassmeier 1987; Pincon and Lefeuvre 1988; Pinçon and Lefeuvre 1991, 1992; Pinçon and Motschmann 1998), Cluster has revealed new three-dimensional properties of space plasma turbulence.

$K$-filtering is a technique designed for multipoint measurements. The technique combines several time series recorded simultaneously at different locations to estimate the energy density in Fourier space as a four-dimensional function, $P(\omega, \mathbf{k})$. The method makes no assumptions as to the underlying physics, but does assume that the time series used are reasonably time stationary and spatially homogeneous. The method can be used on fully coupled electromagnetic wave time series provided the proper constraints (e.g., Faraday's Law) are in place (Tjulin et al. 2005, 2008).

$P(\omega, \mathbf{k})$, the main product of the $k$-filtering technique, has been used in a number of studies and in a number of different ways, including determining three-dimensional dispersion relations in identifying the plasma modes that characterize fluctuations in the magnetosheath (Glassmeier et al. 2001; Sahraoui et al. 2003, 2004), in the 
high latitude magnetic cusps (Grison et al. 2005), in the magnetotail in conjunction with magnetic reconnection (Eastwood et al. 2009; Huang et al. 2010); and in the solar wind. Three-dimensional wavenumber spectra of turbulence have been produced by integrating over the angular frequencies $P(\mathbf{k})=\int P(\omega, \mathbf{k}) d \omega$ in studies of lowfrequency magnetosheath turbulence (Sahraoui et al. 2006), MHD-scale turbulence in the foreshock and solar wind (Narita et al. 2006, 2010), and kinetic-scale turbulence in the solar wind (Sahraoui et al. 2010b). Constantinescu et al. (2006) have used spherical wave decomposition instead of plane waves to locate regions in space where wave generation is occurring.

The $k$-filtering technique has several limitations and caveats that can affect the results and the physical interpretation if they are not understood correctly. A detailed discussion of these points can be found in Sahraoui et al. (2010a) and references therein. Here we discuss briefly the major limitation known as spatial aliasing. In temporal Fourier analysis, if $\delta t$ is the sampling time of the time series, then the highest accessible angular frequency (or the Nyquist frequency) is $\omega_{N}=2 \pi / 2 \delta t$ and all the accessible frequencies belong to $\left[-\omega_{N}, \omega_{N}\right]$. An anti-aliasing filter has to be implemented in the hardware of an experiment to filter higher frequencies and thus suppress the aliasing effect. The resolution $\delta \omega$ in Fourier space, or consequently, the smallest available angular frequency $\omega_{\min }$, is given by $\delta \omega=2 \pi / T$ where $T$ is the total time interval. In practice $T$ is limited by considerations of time stationarity that are generally required in Fourier analyses.

In the $k$-filtering method the approach is similar, however, the major limitation is that space is probed only in a few points (four in the case of Cluster). Therefore a careful definition of the accessible $k$-space is required. By analogy with the temporal Fourier analysis, if the average distance between the satellites is $d$, then the maximum wavenumber available is $k_{\max }=2 \pi / 2 d$. However, contrary to the temporal Fourier transform, spatial aliasing cannot be avoided because there is no way to filter wavenumbers larger than $k_{\max }$. Therefore, one can only assume that the data will include all wavenumbers smaller than $k_{\max }$. Wavelengths shorter than the spacecraft separation $d$ that exist and propagate past the satellites, will generate aliasing, producing spurious energy peaks in the wavenumber domain $\left[-k_{\max }, k_{\max }\right]$. To minimize spatial aliasing one must restrict the investigated frequencies to some range $\left[-\omega_{\max }, \omega_{\max }\right]$ under the assumption that the frequency is generally an increasing function of wavenumber. Given a flow speed $V_{f}$ and fluctuation phase speed $V_{\phi}$ the maximum frequency that can be analyzed using the $k$-filtering is $\omega_{\max }=k_{\max }\left(V_{f}-V_{\phi}\right)$. A detailed discussion of these points can be found in Sahraoui et al. (2010a).

\subsubsection{Electron scale turbulence.}

The high time resolution of the field instruments on Cluster (waveforms of both electric and magnetic field fluctuations are available up to $180 \mathrm{~Hz}$ ) has provided a probe into electron scale turbulence in the solar wind and magnetosphere (Alexandrova et al. 2009; Kiyani et al. 2009; Sahraoui et al. 2009; Sahraoui et al. 2010b). Consequently, it is now clear that the cascade of solar wind turbulence does continue below the ion gyro scale, $\rho_{i}=V_{t h_{i}} / \omega_{c i}$, down to the electron gyro scale, $\rho_{e}=V_{t h_{e}} / \omega_{c e}$, where dissipation becomes important and the spectra steepen $\left(V_{t h_{i, e}}\right.$ and $\omega_{c i, e}$ are the thermal speeds and gyrofrequencies of ions and electrons, respectively). These observations have driven an intensive research into electron scale turbulence both theoretically and numerically (Meyrand and Galtier 2010; Podesta et al. 2010; Camporeale and Burgess 2011a,b; Chang et al. 2011; Howes et al. 2011; Meyrand and Galtier 2012; Sahraoui et al. 2012). However, the 
underlying physics remains controversial, owing to the scarcity of observations and to the fact that theoretical and numerical work exploring electron scale phenomena is all relatively recent. The first point of controversy is the nature of the scaling of the magnetic energy spectra down to and below $\rho_{e}$. Sahraoui et al. (2009) first reported a power-law cascade $f^{-2.8}$ down to $f_{\rho_{e}}$, where a clear spectral break was observed. That break was followed by another power-law-like spectrum that was close to $f_{s c}{ }^{-4}$ (where $f_{\rho_{e}}$ is the frequency in the spacecraft frame corresponding to the electron gyroscale). In a survey of ten years of STAFF waveform data in the free-streaming solar wind Sahraoui et al. (2013) have confirmed those results and showed that the distribution of the slopes below the break frequency, $f_{b}$, is narrow and peaks around -2.8 , while the distribution of slopes above $f_{b}$ is broader and peaks near -4 . On the other hand, Alexandrova et al. (2009), using STAFF-Spectrum Analyzer (STAFF-SA) data, have reported exponential scaling $\sim \exp \left(-\sqrt{k \rho_{e}}\right)$ in the range $k \rho_{e} \sim[0.1,1]$. Alexandrova et al. (2012) proposed that a scaling $k_{\perp}{ }^{-8 / 3} \exp \left(-k_{\perp} \rho_{e}\right)$ best fit the data in the range $k \rho_{e} \sim[0.03,3]$.

A second controversy concerns the identity of the plasma modes that carry the turbulent cascade to electron scales. Two possibilities have been discussed: Kinetic Alfvén Waves (KAW) (Bale et al. 2005; Sahraoui et al. 2009; Sahraoui et al. 2010b; Chen et al. 2013) and whistler turbulence (Podesta et al. 2010). There is growing evidence that the dominant component of the turbulence is the KAW mode. This interpretation that the KAW mode dominates has been demonstrated using estimates of the 'phase speed' $E / B$ (Bale et al. 2005; Sahraoui et al. 2009; Salem et al. 2012), the dispersion relation obtained from the $k$-filtering (Sahraoui et al. 2010b), magnetic compressibility (Kiyani et al. 2013), and from the spectrum of density fluctuations (Chen et al. 2013).

In comparison with solar wind turbulence, turbulence in the magnetosheath has been investigated far less intensively. Indeed, only a handful of studies have been carried out in recent years (see e.g., Sahraoui et al. 2003, 2004; Lacombe et al. 2006; Mangeney et al. 2006; Sahraoui et al. 2006; Alexandrova et al. 2008b; Yordanova et al. 2008; He et al. 2011). Two main similarities with solar wind turbulence emerged from those studies: the turbulence in strongly anisotropic $\left(k_{\|} \ll k_{\perp}\right)$ at subproton and electron scales (Sahraoui et al. 2004; Mangeney et al. 2006; Sahraoui et al. 2006) and kinetic instabilities and nonlinear structures are present (Sahraoui et al. 2004; Sahraoui 2008; Alexandrova et al. 2008b). Major differences with the solar wind do exist, however, e.g., (i) magnetosheath turbulence evolves in a 'confined' space limited by the bow shock and the magnetopause and these boundaries may influence the anisotropy of the turbulence (Sahraoui et al. 2006; Yordanova et al. 2008), and, (ii) in contrast to the solar wind, the fluctuations are dominated by zero-frequency compressible fluctuations (e.g., mirror modes Sahraoui et al. 2006). In a recent large survey of STAFF waveform data obtained in the magnetosheath, Huang et al. (2014) quantified the differences in spectral slopes found in the solar wind and magnetosheath in the magnetic energy spectra between the ion and electrons scales (see Fig. 20). Their analysis revealed considerable similarities. Considering the differences in the plasma parameters in the two regions, which may give rise to different plasmas modes, this result is rather surprising and suggests that the scaling of turbulence does not depend on the local plasma conditions. On the other hand, the slopes at the electron break in the two regions were generally steeper in the magnetosheath where the distribution peaks near -5.5 and slopes can be as steep as -7 . A possible explanation of this discrepancy was suggested by Huang et al. (2014) based on the differences in the 

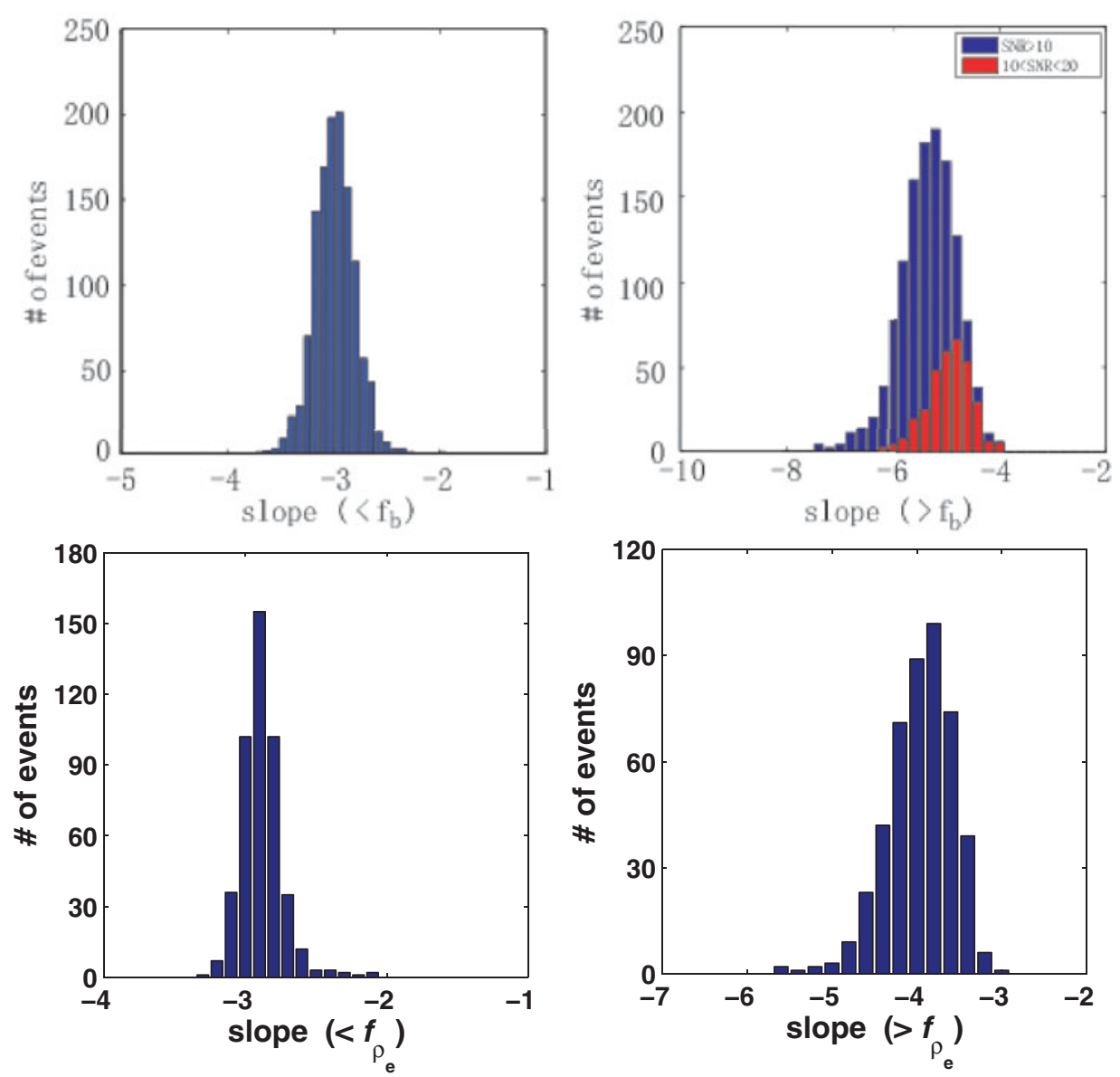

FIGURE 20. Comparison between the scaling of the magnetic energy spectra below and above the electron spectral break, as observed in the magnetosheath (top) and in the SW (bottom) (adapted from Sahraoui et al. 2013; Huang et al. 2014).

SNRs (Signal to Noise Ratios) between the two regions. The nature of the plasma modes dominating at sub-proton scales in the magnetosheath is still an open question and requires deeper statistical investigation using large data samples.

\subsubsection{Vorticity.}

Several studies (see, for example, Matthaeus 1982; El-Alaoui et al. 2010; Palermo et al. 2011) have indicated that vorticity $(\nabla \times \mathbf{V})$ should be ubiquitous in space plasmas. Vorticity can be indicative of a number of phenomena including local turbulence (Kintner and Seyler 1985; Sundkvist et al. 2005), shear flows (Tajima et al. 1991; Rankin et al. 1997), magnetic bubbles (Birn et al. 2004), and reconnection (Matthaeus 1982; Matthaeus and Lamkin 1985; Hwang et al. 2013). It was only with the launch of Cluster that the ability to measure directly local vorticity became possible (albeit only by using moments of the electron distribution function) by computing the spatial variation in the velocity within the volume defined by the Cluster spacecraft (see Sec. 1.4). Enhancements in vorticity have been noted both in the inner plasmasheet (Gurgiolo et al. 2011) and upstream of the bow shock (Gurgiolo et al. 2010). 
In the inner plasmasheet Gurgiolo et al. (2011) found significant vorticity in all of the crossings looked at. Included were three crossings of IDRs associated with magnetic reconnection (Eastwood et al. 2010). Enhanced vorticity was found to encompass each crossing of an IDR. Figure 21, adapted from Gurgiolo et al. (2011), shows the data across one plasmasheet crossing. Plotted from top to bottom are the electron density, parallel velocity, temperature, the velocity divergence and vorticity, and the magnetic field in spherical Geocentric Solar Magnetospheric (GSM) coordinates. The vertical blue lines in the vorticity panel delimit a pair of IDRs, the first of which was studied in detail by Hwang et al. (2013). Both crossings are accompanied by an increase in vorticity that is probably a manifestation of the field-aligned and counter-streaming electrons that surround the events. The association of vorticity with reconnection confirms what is seen in many simulations. Perhaps even more interesting in the figure is the third and last region of enhanced vorticity. Close examination shows a similar velocity profile during this time period to that seen in the previous two enhancements and yet there was no identified crossing of a diffusion region, perhaps because the spacecraft passed just outside an IDR or were close to, or traveled through, a magnetic bubble.

Bursts of vorticity in the solar wind and foreshock have been reported by Gurgiolo et al. (2010). In the solar wind the source of the vorticity is not completely understood, but the authors suggest that it is due in part to a response of the solar wind to small scale magnetic field rotations and fluctuations within the spatial volume enclosed by the spacecraft. They concluded from their analysis that the measure vorticity was driven by either the strahl and/or particles reflected from the foreshock.

As both the strahl and the return populations are field-aligned and therefore react to changes in the field orientation an attempt was made to correlate changes in vorticity with changes in the orientation of the magnetic field. They concluded that, to a large extent, the vorticity is formed by a combination of the strahl and return electrons and the response of those populations to variations in the orientation of the magnetic field. Another source of vorticity is the velocity shear that is setup along the foreshock boundary that separates regions of returning and non-returning electrons.

Vorticity is expected in the magnetosheath due to, for example, reconnection along the dayside magnetopause (Lockwood and Smith 1992; Retinò et al. 2007; Dunlop et al. 2011) and along the flanks (Lin and Lee 1994), as well as due to velocity shear along the magnetopause/magnetosheath boundary (Hasegawa et al. 2004; Hasegawa et al. 2009). Figure 22 shows an interval of Cluster data when the spacecraft were in a high inclination orbit near apogee, just crossing the northern magnetopause and entering the magnetosheath. The spectrogram of the energy flux in the upper panel shows data from one of the central PEACE sensors. The red band centered just below $100 \mathrm{eV}$ is a general signature of the magnetosheath. Variations are due to boundary motions as the spacecraft passed in and out of the LLBL and magnetosheath (the higher energy and temperatures (bottom panel) are indicators of the LLBL). The drop out centered at 14:43 UT is the result of a momentary shift in the magnetic field from southward to northward seen in the ACE magnetic field data (not shown). The second panel shows the measured vorticity. There are multiple enhancements across the time period. Almost all occur in conjunction with boundary crossings. However, there is little vorticity between 14:20 and 14:40 UT when the spacecraft are in the magnetosheath and away from the boundary shear flows. 


\section{Cluster C1 Overview Plots}

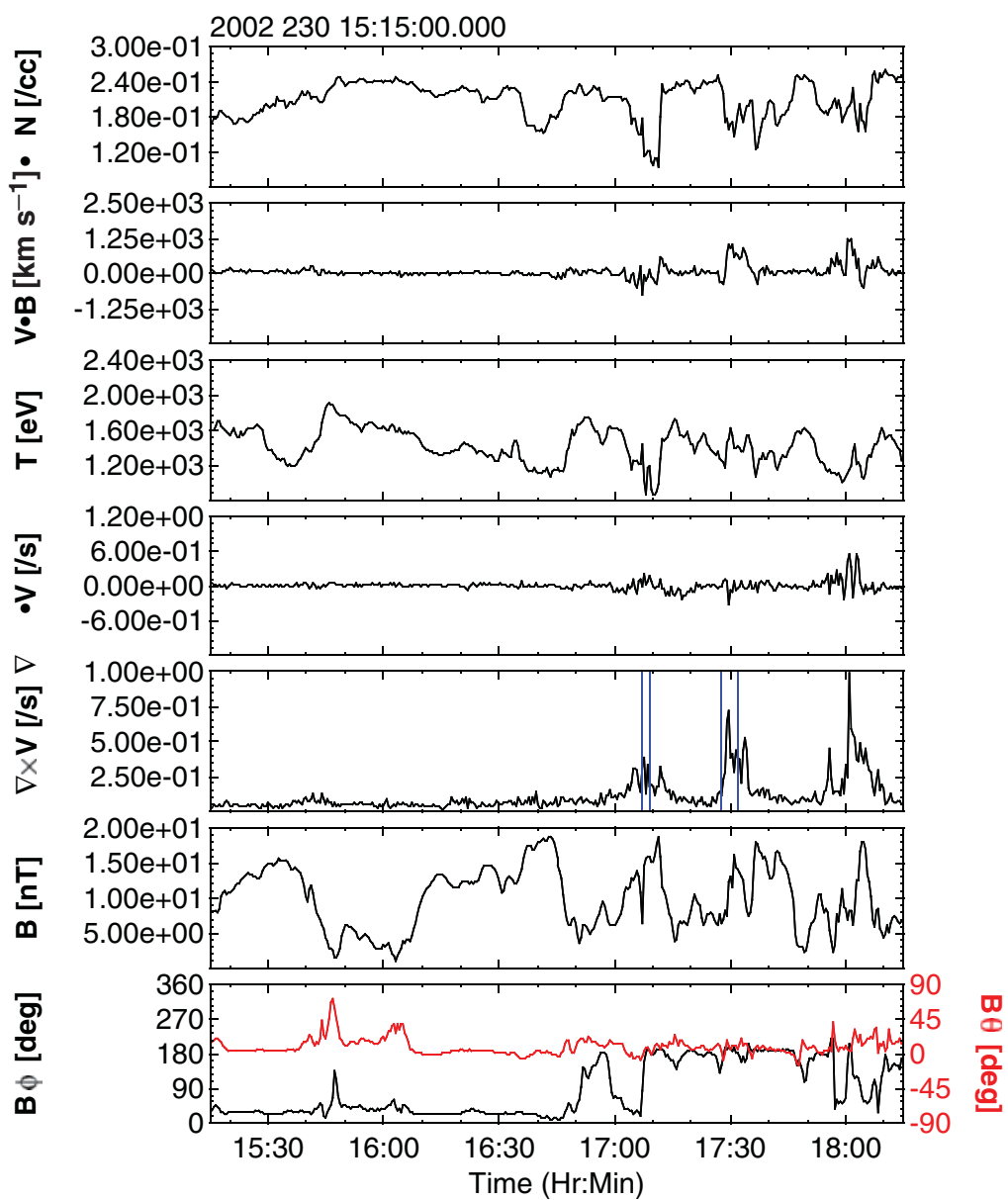

FIGURE 21. Figure adapted from Gurgiolo et al. (2011) showing regions of enhanced vorticity during a crossing of the inner plasmasheet. Vertical blue lines delineate two crossings of a reconnection ion diffusion region.

\subsubsection{Dissipation - magnetic discontinuities and intermittency.}

Discontinuities are often present in both the solar wind and magnetosphere. They may represent the borders in between 'spaghetti-like' magnetic flux tubes of a discontinuous (solar wind) plasma (Burlaga 1969; Bruno et al. 2001; Borovsky 2006), or, again in the solar wind, may be fossils of the solar coronal origin (Burlaga 1968; Borovsky 2006). In addition, observed discontinuities often coincide with current sheets that may form as a consequence of an MHD turbulent cascade (Matthaeus and Montgomery 1980; Veltri 1999) and recently, it has been pointed out that their statistical properties are similar to distributions obtained from numerical simulations of MHD turbulence (Greco et al. 2008, 2009). In this interpretation, current sheets are characteristic coherent structures expected in active intermittent MHD turbulence and are therefore integral to the dynamical couplings across scales. It is quite possible that discontinuities are related to both flux tube boundaries and intermittent structures that appear spontaneously in MHD turbulence (Matthaeus and Montgomery 1980; Matthaeus et al. 1986b; Carbone et al. 1990; Veltri and Mangeney 1999; Vasquez 


\section{Cluster C1 Overview Plots}
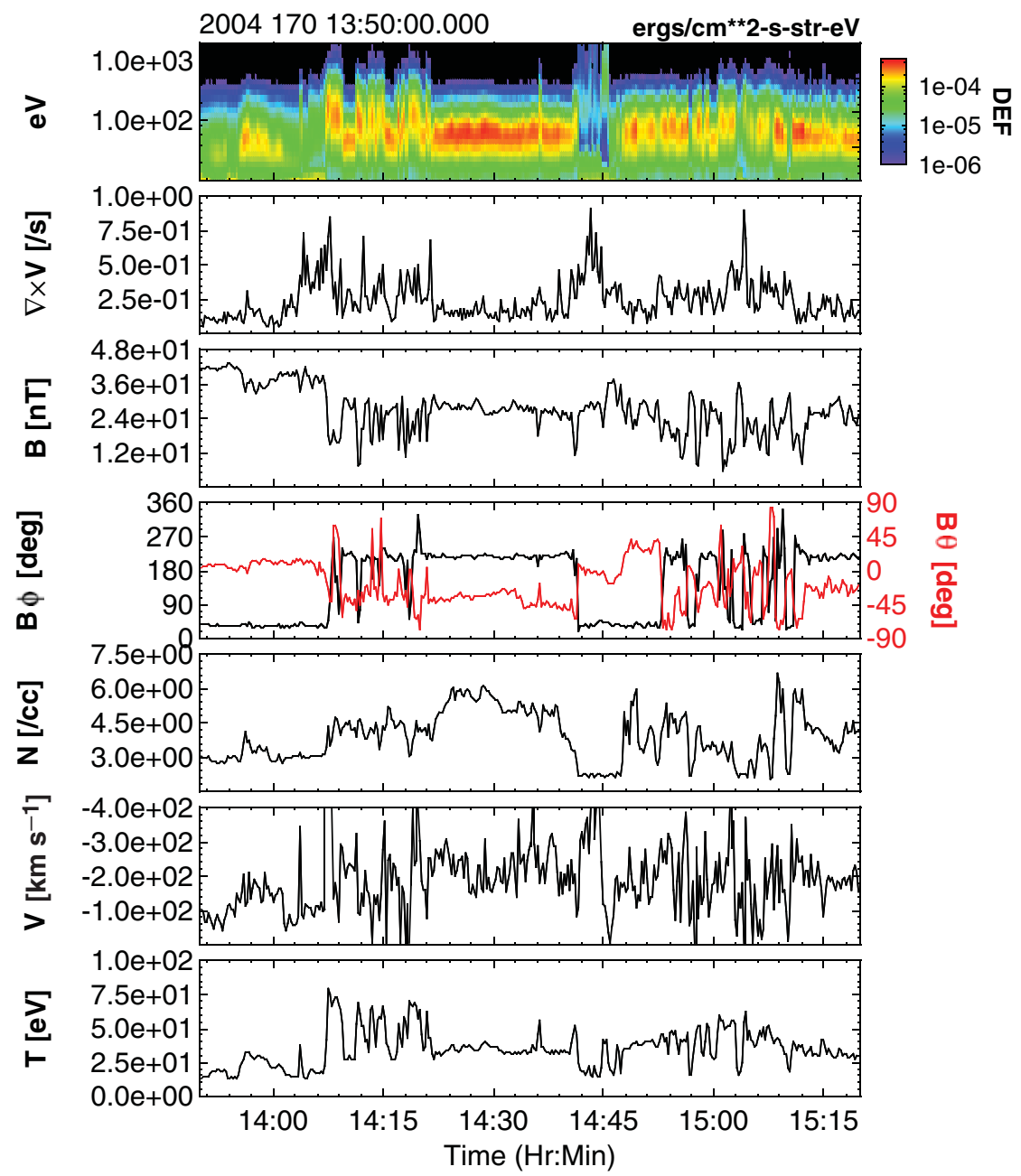

FIGURE 22. Cluster passage into the northern magnetosheath showing bursts of vorticity mainly aligning with boundary crossings.

et al. 2007; Servidio et al. 2008). Intermittent fluctuations have been associated with enhanced turbulent dissipation and non-uniform plasma heating (Osman et al. 2011; Servidio et al. 2012; Wan et al. 2012; Perrone et al. 2013; Wu et al. 2013).

There is evidence suggesting that increased helium, proton and electron temperature anisotropies are linked to near-discontinuous structures. The same structures can cause deviations from local thermal equilibrium in velocity distribution functions and have been found in plasmas unstable to microinstabilities (Bale et al. 2009). Recent work has suggested that such structures contribute to the acceleration and transport of supra-thermal particles (Tessein et al. 2013). Relationships must exist, therefore, between coherent structures, intermittent turbulence, plasma heating and broader kinetic activity. Another interesting link exists between coherent structures and magnetic reconnection. Multispacecraft observations have revealed the presence of current sheets in the magnetosheath that show evidence of magnetic reconnection 

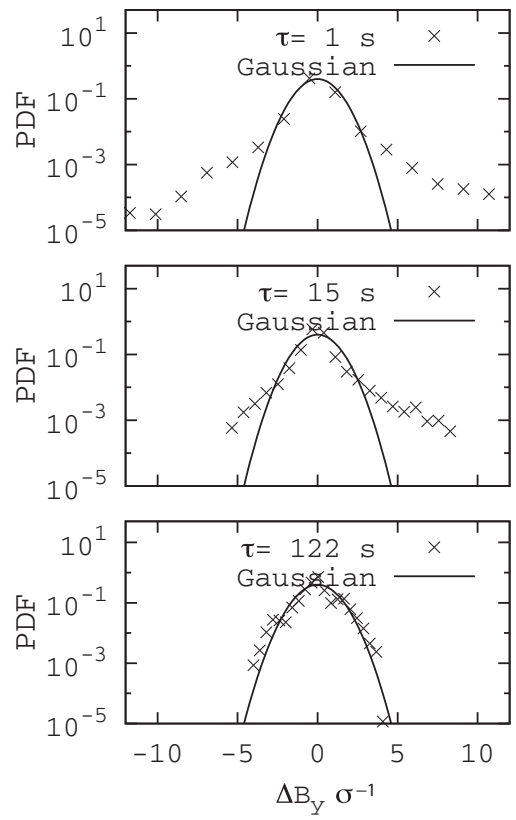

FIGURE 23. PDFs of $B$ increments in (2.4), for $B_{y}$ normalized to its standard deviation. The increments have been evaluated at: $\tau=122 \mathrm{~s}$ (bottom panel), $15 \mathrm{~s}$ (middle panel), and 1s (top panel). A Gaussian distribution (thick-solid black) is shown for comparison. For smaller $\tau$, the distribution develops fat tails. Data are from FGM on C2 during a period of pristine solar wind on 2004-01-10 (06:05:00 UT to 07:00:00 UT), during which Cluster was not connected to the electron foreshock.

(Retinò et al. 2007), and also have the characteristics of intermittent turbulence (Sundkvist et al. 2007).

\subsubsection{Analysis of discontinuities.}

Using Cluster datasets we review some of the main results about solar wind discontinuities and intermittency, showing examples for both the MHD and kinetic (small-scale) regimes. In plasma turbulence, intermittency can be investigated through the analysis of the magnetic field increments

$$
\Delta B_{i}(t, \tau)=B_{i}(t+\tau)-B_{i}(t),
$$

where the index $i$ refers to the magnetic field component, $t$ is the time, and $\tau$ is the time-lag (which generally in the solar wind runs from hours to fractions of a second.) The dataset described here (and studied in other contexts by Sahraoui et al. (2010b) and by Perri et al. (2012)) represents a quasi-stationary solar wind period. The interval is taken on 2004-01-10, from 06:05:00-07:00:00 UT, during time which Cluster was not connected to the electron foreshock. The solar wind had an average plasma density of $n_{p} \sim 15 \mathrm{~cm}^{-3}$ and a mean speed of $V \sim 550 \mathrm{~km} \mathrm{~s}^{-1}$ (Sahraoui et al. 2010b). The $67 \mathrm{vec} \mathrm{s}^{-1}$ resolution data come from the FGM experiment on C2 (Balogh et al. 1997).

Figure 23 displays the probability density functions (PDFs) of the magnetic field increments, described by (2.4), normalized to the their standard deviation, for the $y$-component of the magnetic field. Different components have similar statistical behavior (not shown here). Data used are in GSE coordinates. Increments have been calculated using a time lag within the inertial or Kolmogorov range of magnetic 


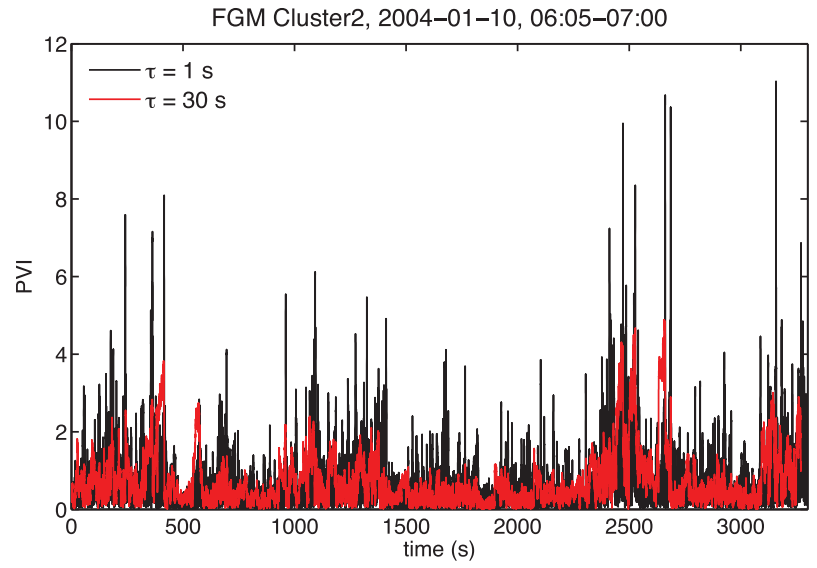

Figure 24. PVI time-series at two different time lags, $\tau=30 \mathrm{~s}$ (red) and $1 \mathrm{~s}$ (thick black). The degree of burstiness of the signal increases as $\tau$ decreases, typical of intermittency. The strongest events may correspond to discontinuities and/or sites of reconnection.

turbulence. As $\tau$ decreases (from bottom to top in Fig. 23), the PDFs develop higher tails, clearly deviating from the Gaussian distribution (thick solid black line. The most intermittent time-scale is $1 \mathrm{~s}$ and is clear evidence for generation of small scale, non-homogeneous, structures in plasma turbulence (Marsch and Tu 1997; SorrisoValvo et al. 1999). Intermittency at the MHD scales has also been observed in other Cluster datasets (Alexandrova et al. 2008a; Kiyani et al. 2009), and appears to be a fundamental feature of the cascade.

Greco et al. (2008) proposed another method for investigating the intermittent behavior of solar wind turbulence using the Partial Variance of Increments (PVI) technique. This method analyzes the signal

$$
\operatorname{PVI}(t, \tau, T)=\frac{|\Delta \mathbf{B}(t, \tau)|}{\sqrt{\left\langle|\Delta \mathbf{B}(t, \tau)|^{2}\right\rangle}},
$$

where $\Delta \mathbf{B}(t, \tau)$ are the magnetic field (vector) increments in (2.4), and the brackets $\langle\bullet\rangle_{T}$ represent a time average. This method is able to detect rapid changes in the magnetic field vector and is very efficient in selecting abrupt discontinuities (Greco et al. 2009; Greco and Perri 2014). To satisfy statistical convergence, the averaging time $T$ should be bigger than (or on the order of) the correlation time of turbulence $\tau_{c}$. For typical parameters of the solar wind, the correlation time varies between mostly $40 \mathrm{~m}$ and a couple of hours (Jokipii and Hollweg 1970; Matthaeus et al. 1986a, 2005; Bruno and Carbone 2005; Matthaeus et al. 2005). In our specific case, the average has been taken over the entire data set, $T=55 \mathrm{~min}$, which is somewhat larger than a correlation time. The PVI signal has routinely been used in both numerical simulation analysis of intermittency, as well as in laboratory and solar wind datasets (Wang et al. 2013; Schaffner et al. 2014). In addition, coherent structures have also been identified using other approaches, such as wavelets (Veltri and Mangeney 1999; Bruno et al. 2001), phase coherency analysis (Hada et al. 2003; Koga et al. 2007) and 'surrogate data' analysis (Sahraoui 2008; Sahraoui and Goldstein 2010).

Figure 24 shows an example of PVI time series at two different time lags as computed from Cluster data. The signals appear burst-like with large amplitude spikes, which suggest the presence of sharp gradients and localized coherent structures in the magnetic field that represent the spatial intermittency of turbulence. Notice 


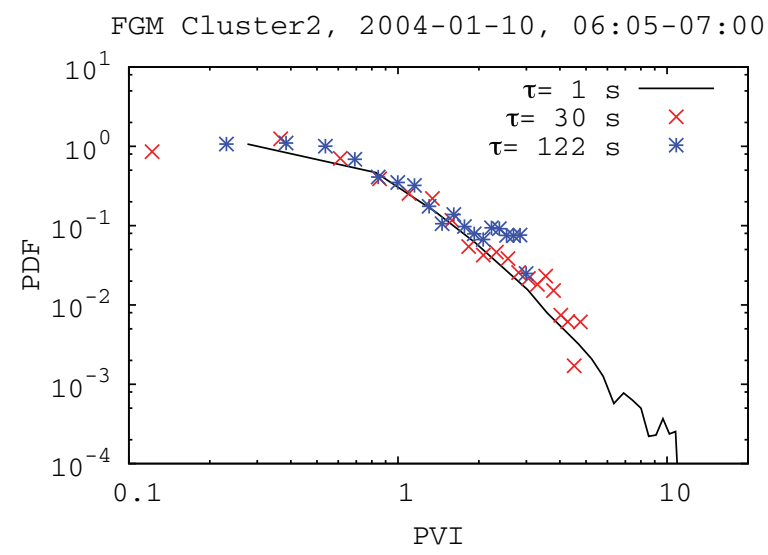

FIGURE 25. PDFs of the PVI signals of Fig. 24 at $\tau=122 \mathrm{~s}$ (blue stars), $\tau=30 \mathrm{~s}$ (red X's), and $\tau=1 \mathrm{~s}$ (black line). For small $\tau$ 's the distribution is broader, reaching PVI $>10$, indicating the presence of strong non-Gaussian events.

that the degree of burstiness becomes lower as $\tau$ increases. The distributions of the two PVI signals are compared in Fig. 25: the PDFs are very broad and for the smaller time-scale, namely $\tau=1 \mathrm{~s}$ (black line), the tail of the distribution is more extended, indicating a higher probability of occurrence. These strong PVI values, reaching PVI $>10$, are typical of solar wind intermittency.

By imposing a threshold on (2.5), it is possible to select the largest amplitude events, which are likely to be either discontinuities or reconnection events. An example of an above threshold PVI event is shown in Fig. 26. The magnetic field components have been rotated into the local minimum variance (MV) reference frame (Sonnerup and Scheible 1998; Perri et al. 2009) (top panel in Fig. 26). This rotation has been done by computing the eigenvalues and the eigenvectors of the one-point correlation matrix, i.e.,

$$
S_{i j}=\left\langle B_{i} B_{j}\right\rangle-\left\langle B_{i}\right\rangle\left\langle B_{j}\right\rangle,
$$

where $i$ and $j$ indicates the magnetic field components in a generic reference frame and $\langle\ldots\rangle$ is a time average. The eigenvectors of the matrix in (2.6) form the MV reference system having one of the axis aligned along the direction of minimum variation of the magnetic field fluctuations. In the event analyzed (see Fig. 26), the MV direction (solid red line) is steady state and very close to zero, along the maximum variance direction (short-dashed blue line) the magnetic field exhibits a large amplitude rotation (about $180^{\circ}$ ). During this event the magnetic field magnitude undergoes a decrease (bottom panel). It is worth stressing that the eigenvalues of the MV matrix computed for this discontinuity are such that $\lambda_{\max }>\lambda_{\text {med }} \gg \lambda_{\text {min }}$. For example, in Fig. 26 , the values are $\left(\lambda_{\text {max }}, \lambda_{\text {med }}, \lambda_{\text {min }}\right)=(15,1.3,0.07)$, indicating the presence of strongly anisotropic fluctuations. Note that this discontinuity shows many similarities with the reconnection events commonly observed in simulations and in the solar wind exhausts (Gosling et al. 2005; Gosling and Szabo 2008; Servidio et al. 2009).

Beyond the inertial range, Sahraoui et al. (2009); Kiyani et al. (2009); Alexandrova et al. (2009); Sahraoui et al. (2010b); Perri et al. (2012) used STAFF data to further investigate the presence of magnetic discontinuities from proton to electron scales in the dissipation range. The solar wind time intervals were burst mode data at 450 $\operatorname{vec~s}^{-1}$ resolution, which has allowed analysis of solar wind turbulence at very high 


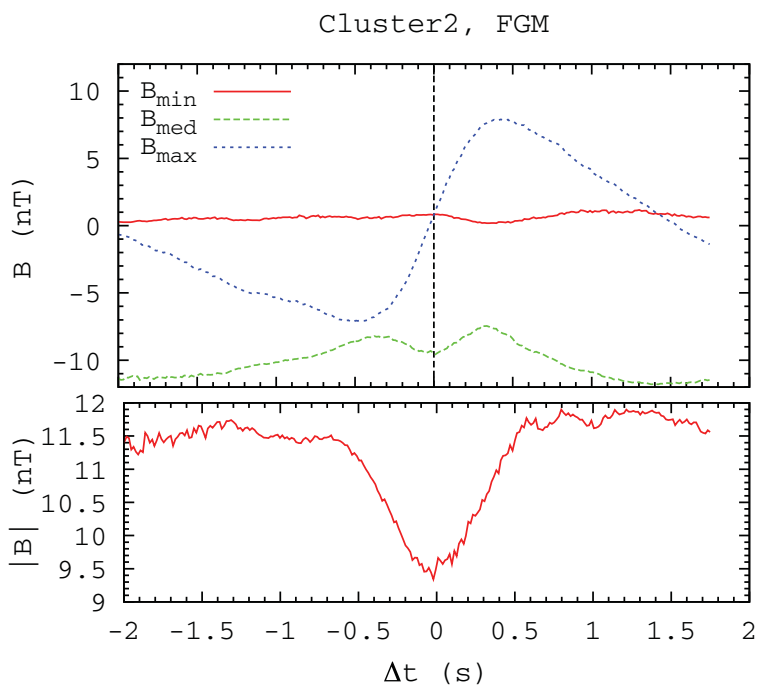

FIGURE 26. Example of a discontinuity identified with the PVI technique. The components of $\mathbf{B}$ are displayed in the minimum variance reference frame (top panel). The $|B|$ is shown in the bottom panel. The vertical black dashed line indicates the time of the discontinuity. All are plotted as a function of the time difference between the instantaneous time $t$ and the time of occurrence of the discontinuity, $t_{0}$, i.e., $\Delta t=t-t_{0}$.

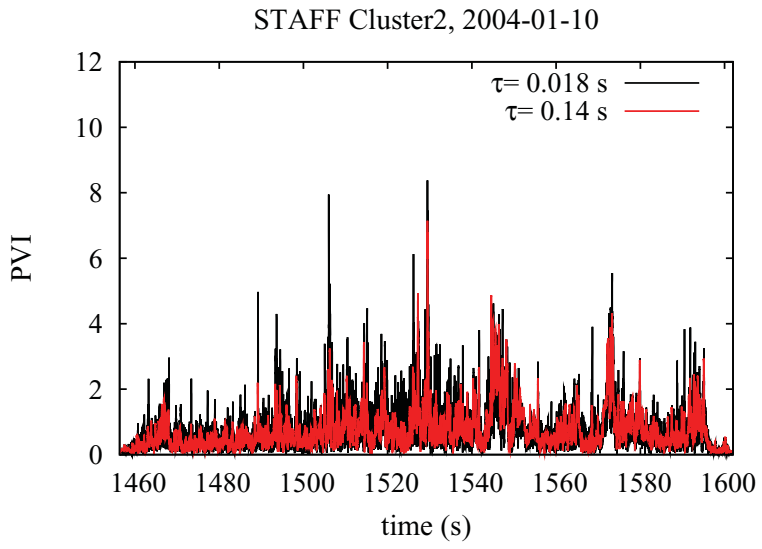

FIGURE 27. PVI time-series at time lags, $\tau=0.14 \mathrm{~s}$ (red) and $0.018 \mathrm{~s}$ (thick black), computed using burst mode data from STAFF on C2. Time refers to seconds after 06:05:00 UT.

frequencies. Figure 27 displays the time series of the PVI computed from the STAFF data at $\tau=0.018 \mathrm{~s}$ (solid black line) and $\tau=0.14 \mathrm{~s}$ (dotted red line). A comparison with Fig. 24, i.e., PVI time series in the inertial range, shows that the signals are very burst like, albeit the amplitude is slightly lower. This is due in part to the fact that, at smaller scales, the magnetic energy is lower, and in part to the fact that the denominator in (2.5) has been computed over shorter time scales. Using the PVI signal at $\tau=0.018 \mathrm{~s}$, we have selected those bursts whose amplitude is above a given threshold. In particular, Fig. 28 shows a discontinuity characterized by PVI $>2$. As for the discontinuity reported in Fig. 26, the eigenvalues of the MV matrix are such that $\lambda_{\text {max }} \gg \lambda_{\text {med }}>\lambda_{\text {min }}$, thus the three directions are well defined. Along the maximum variance direction the field rotates of about $180^{\circ}$, the $\mathrm{MV}$ direction is 


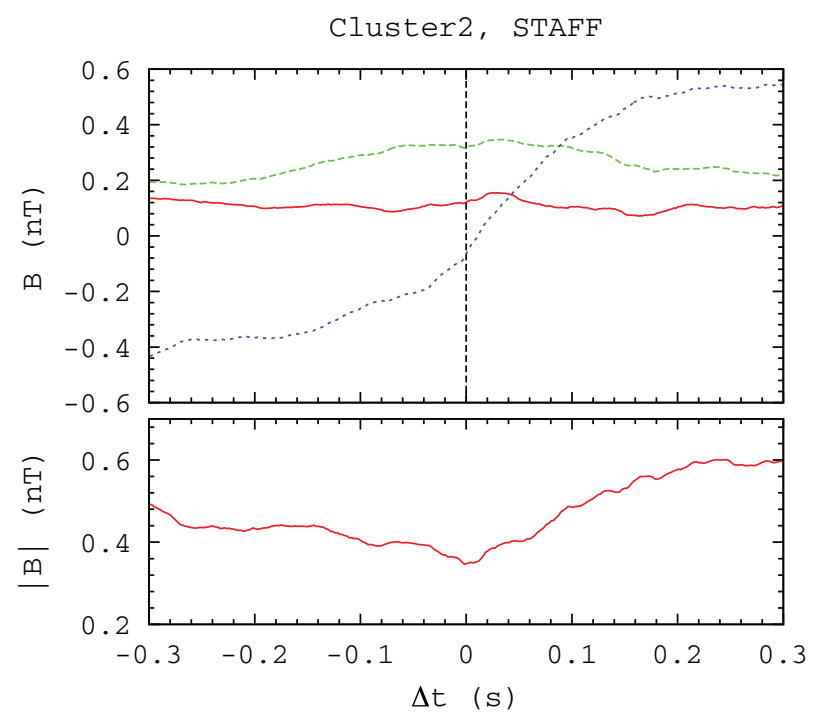

Figure 28. Same as Fig. 26 but for a discontinuity identified using PVI (computed at $\tau=0.018$ s) on STAFF data. The components of B are in the minimum variance reference frame (top panel). $|B|$ is shown in the bottom panel. The vertical black dashed line locates the time of the discontinuity.

close to the zero value, and the magnetic field magnitude (bottom panel) slightly decreases at the discontinuity. This event spans a time scale $\tau \sim 0.4 \mathrm{~s}$, therefore, it is well within the high frequency range of magnetic solar wind turbulence. This event is an example of the presence of very thin discontinuities (and/or current sheets) that might be sites of magnetic energy dissipation (Perri et al. 2012). Moreover, this further confirms that turbulence proceeds at smaller scales generating coherent and discontinuous structures.

\subsubsection{Taylor microscale.}

There are two approaches that have been used to estimate the Taylor microscale $\left(\lambda_{\mathrm{T}}\right)$ in space plasmas, both of which give nearly equivalent results. While determining $\lambda_{\mathrm{T}}$ is fairly routine in fluid turbulence (Kailasnath and Sreenivasan 1993; Belmabrouk and Michard 1998; Segalini et al. 2011), due to limitations in the time resolution of plasma instruments and the limited number of multi-spacecraft data sets, $\lambda_{\mathrm{T}}$ was not evaluated in space plasmas prior to the work of Matthaeus et al. (2005). Under the assumption of isotropy, the approach used consisted of estimating the radius of curvature of the correlation function $R(r)=\langle\alpha(x) \cdot \alpha(x+r)\rangle$ in the limit of small $r$, i.e.

$$
R(r) \approx\left\langle\alpha^{2}\right\rangle\left(1-\frac{r^{2}}{2 \lambda_{T \alpha}^{2}(r)}\right)+\cdots
$$

where $\alpha$ is the fluctuating component of the physical quantify being used to estimate $\lambda_{T \alpha}$ ( $B$ for magnetic field and $V$ for velocity) and $r$ is the spacecraft separation if using data from the two spacecraft, or the spatial lag when using data from a single spacecraft under the assumption that the Taylor frozen-in-flow hypothesis (Taylor 1938 ) is valid. To remove the $r$ dependence the computation is performed over a wide range of separations and the total ensemble of results is interpolated to $\lambda_{\mathrm{T} \alpha}(0)$. Weygand et al. $(2007,2009,2010,2011)$ have used this method to explore the size 
and symmetry properties of $\lambda_{\mathrm{TB}}$ in both the solar wind and plasmasheet, obtaining estimates of $\lambda_{T B}$ in the solar wind of $2400 \pm 100 \mathrm{~km}$ and in the plasmasheet of $1900 \pm 100 \mathrm{~km}$.

The second approach (Gurgiolo et al. 2013) estimates $\lambda_{T \alpha}$ by evaluating the exact formulation (see Taylor 1935; Batchelor 1953):

$$
\lambda_{T \alpha}=\sqrt{\frac{\left\langle\alpha^{2}\right\rangle}{\left\langle(\nabla \times \alpha)^{2}\right\rangle}} .
$$

This approach could not be used prior the launch of Cluster (see Sec. 1.4).

There are two major obstacles to using (2.8). The first is to ensure that the estimate of $\lambda_{T}$ is made out to the correlation scale and the second is to remove the dependence on spacecraft separation from the spatial derivatives used to estimate the curl. The requirement that the spacecraft be in a near perfect tetrahedral configuration often restricts the interval of time that can be used in each solution of (2.8), which in turn limits the scale length to values below the correlation scale. This can be compensated for by removing the mean field, which is done by applying a low-pass Savitzky-Golay filter to the data and then subtracting the filtered from the unfiltered data leaving the fluctuating component. The low-pass filter frequency sets the scale length over which the data is processed as:

$$
\lambda=V_{s w} / f
$$

where $\lambda$ is a scale length, $V_{s w}$ is the solar wind speed and $f$ is the low-pass cutoff frequency.

Gurgiolo et al. (2013) showed that by computing $\lambda_{T}$ for a number of low-pass cutoff frequencies, $\lambda_{T}$ can easily be interpolated out to the correlation scale $\left(\sim 2 \times 10^{6} \mathrm{~km}\right)$. An example of the relationship between $\lambda_{T}$ and the filter frequency is shown in Fig. 29 where $\lambda_{T}$ is plotted as a function of cutoff frequency (lower $x$-axis) or equivalently scale length (upper $x$-axis). $\lambda_{T}$ was estimated at eight equally logarithmically spaced cutoff frequencies ranging from $0.03125-0.001 \mathrm{~Hz}$ (equivalent to computing $\lambda_{T}$ between the scale lengths $1.6 \times 10^{5}-5 \times 10^{5} \mathrm{~km}$ ). The line is an exponential fit to the data and can be used to interpolate $\lambda_{T}$ out to the correlation scale.

The analysis above needs to be done for a large number of time intervals spanning a range of spacecraft separations $(\triangle d)$ which provides the data set $\lambda_{T}(\mathrm{~Hz}, \triangle d)$. Fits to plots of $\lambda_{T}(\mathrm{~Hz}, \triangle d)$ versus $\triangle d$ for each filter frequency are used to remove the $\triangle d$ dependence giving $\lambda_{T}(\mathrm{~Hz}, \triangle d=0)$. A further fit of the ensemble $\lambda_{T}(\mathrm{~Hz}, \triangle d=0)$ against frequency allows $\lambda_{T}$ to be estimated at the correlation scale. This is shown in the two plots in Fig. 30. The upper and lower plots show fits to $\lambda_{T B}(\mathrm{~Hz})$ and the corresponding errors as a function of filter frequency. The fits give a value for $\lambda_{T B}$ at the correlation scale of $1538 \pm 550 \mathrm{~km}$, which can be compared to the value of $2400 \pm 100 \mathrm{~km}$ obtained by Weygand et al. (2007). The difference between the two estimates may be a consequence of the presence of anisotropy in the local turbulence (Weygand et al. 2011).

Theoretically if also should be possible to compute $\lambda_{T V}$ from the electron velocity, however, there were not enough data intervals to provide adequate statistics. Unlike magnetic field data, 4-s resolution (electron) velocity data from the four Cluster spacecraft are available only when the spacecraft are in burst mode, as that provides a continuous set of $3 \mathrm{D}$ velocity distribution from which the moments can be calculated. However, relative estimates of $\lambda_{T V}$ can be made for individual intervals. Comparing these to $\lambda_{\mathrm{TB}}$ values estimated from magnetic field data for the same time interval using the same spacecraft separation showed that estimates of $\lambda_{T V}$ were consistently 


\section{Cluster MF Taylor Microscale Estimates \\ Based on 0.02s Magnetic Field Data}

2003051 06:15:00.000 TO 2003051 06:45:00.000

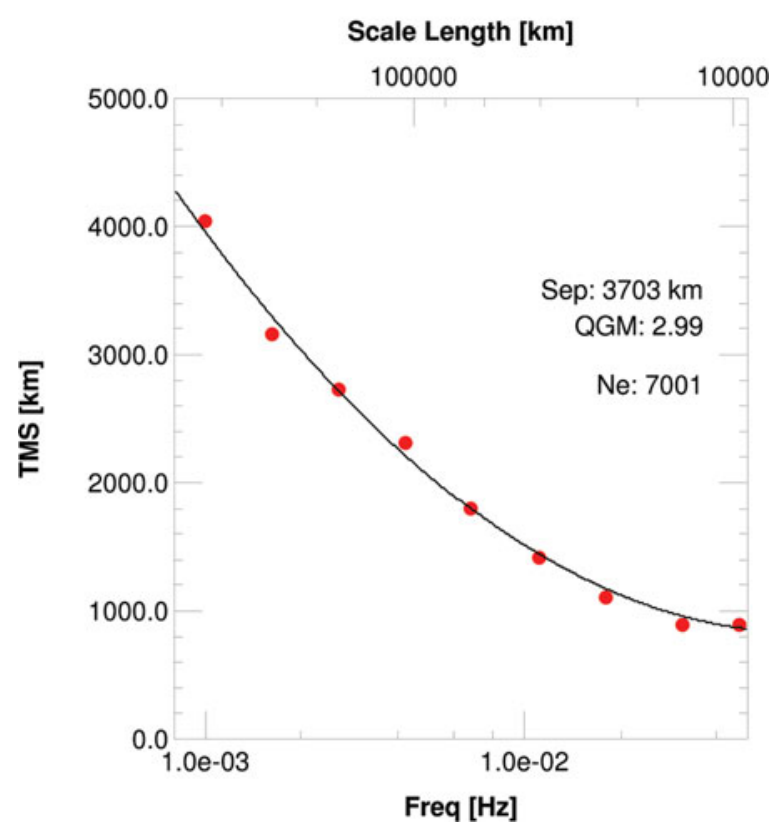

FIGURE 29. The dependence of $\lambda_{T B}$ on the filter frequency used to remove the mean for 9 equally logarithmically spaced frequencies. The equivalent scale lengths are shown on the upper $\mathrm{X}$ axis. An exponential fit to the data allows the $\lambda_{T B}$ to be interpolated back to the correlation scale. (From Gurgiolo et al. 2013.)

lower than the corresponding estimates of $\lambda_{T B}$. The reason for this is not well understood.

\section{Summary and conclusions}

We have highlighted some of the more important contributions Cluster has made that have increased understanding of physical processes active within the magnetosphere and near-Earth interplanetary space. Much of the work discussed above is unique to Cluster and required concurrent multi-spacecraft observations within various restricted regions and/or the very high time resolution of the WBD and STAFF experiments. By no means have we covered all of the important discoveries reported by the Cluster teams. Processes we've discussed above include:

- aspects of magnetic reconnection as elucidated by the multispacecraft instrumentation on Cluster-multiple x-points, proximity to the EDR, magnetic nulls, properties of dipolarization events associated with reconnection, acceleration mechanisms, etc.

- shear-flow excitation of large-scale waves and vortices via the KHI.

- discoveries related to plasma excitation of high-frequency radio waves including new details on the properties of AKR, chorus, ESWs, hiss and magnetosonic solitons.

- determination of the thickness of the Earth's collisionless bow shock.

- investigation how entropy is affected as the plasma flows from up to downstream across the shock. 

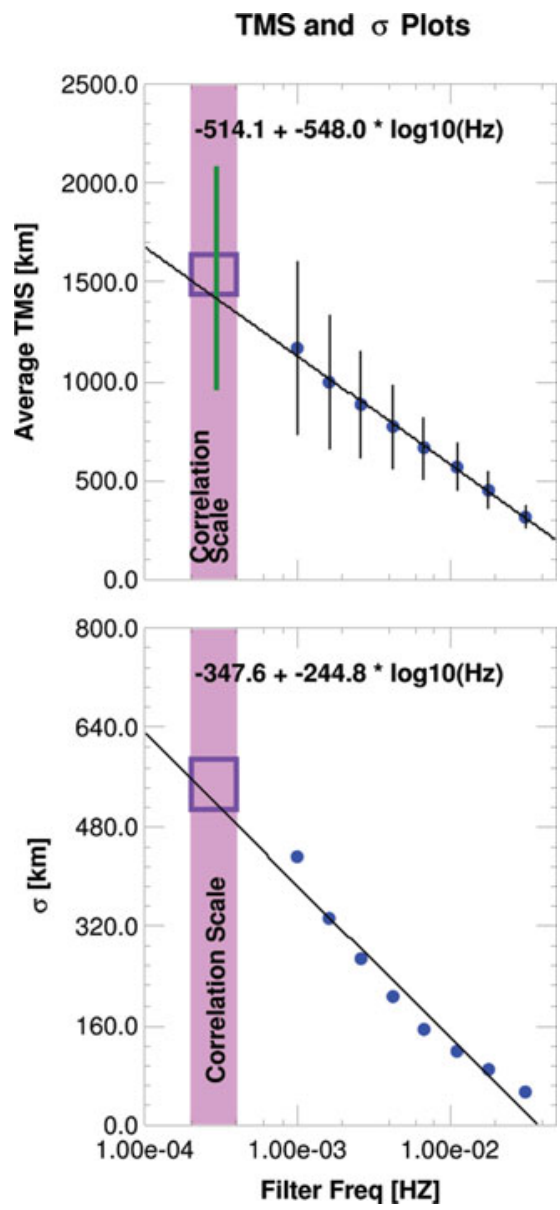

FIGURE 30. Interpolation of spacecraft separation corrected $\lambda_{T B}$ and its corresponding deviation out to the correlation scale (purple band). Analysis was done using $0.2 \mathrm{~s}$ resolution magnetic field data. (From Gurgiolo et al. 2013.)

- characterizing the non-thermal electron distribution function of the solar wind core, halo, super-halo and strahl.

- using the four spacecraft to determine currents and vorticity in various regions of geospace.

- determining the turbulence correlation scale in the solar wind and plasmasheet.

- measuring the Taylor microscale in the solar wind.

- describing for the first time the three-dimensional properties of the inertial range of interplanetary turbulence at ion scales.

- using high-resolution magnetic field data to study turbulence at electron scales at which the plasma dissipates, discontinuities form and intermittency manifests itself.

Studies of space plasmas have matured to the point that many further advancements will be dependent on multi-spacecraft observations, such as those provided by Cluster and soon to be provided by MMS. Multiple spacecraft mission coupled with higher time resolution instruments will allow research to ever smaller scale lengths. 


\section{Acknowledgements}

The authors would like to thank the Cluster experiment teams for making available their data. J. S. Pickett, S. Fung, G. K. Parks, and M. L. Goldstein would like to acknowledge the support of NASA headquarters to the Cluster mission. A. F. Viñas, D. E. Wendell, K.-J. Hwang, and MLG also acknowledge support from the MMS Interdisciplinary Science Team at the Goddard Space Flight Center for its support. J. M. Weygand was supported, in part, by NSF grant AGS-1155841, and S. Servidio acknowledges support by the project POR Calabria FSE 2007/2013 and the Marie Curie Project FP7 PIRSES-2010-269297 'Turboplasmas'.

\section{REFERENCES}

Alcaydé, D. 2001 Editorial. Ann. Geophys. 19(10/12), 1195-1195.

Alexandrova, O., Carbone, V., Veltri, P. and Sorriso-Valvo, L. 2008a Small scale energy cascade of the solar wind turbulence. Astrophys. J. Lett. 674, 1153-1157.

Alexandrova, O., Lacombe, C. and Mangeney, A. 2008b Spectra and anisotropy of magnetic fluctuations in the Earth's magnetosheath: cluster observations. Ann. Geophys. 26, 3585-3596.

Alexandrova, O., Lacombe, C., Mangeney, A., Grappin, R. and Maksimovic, M. 2012 Solar wind turbulent spectrum at plasma kinetic scales. Astrophys. J. 760(2), 121.

Alexandrova, O., Saur, J., Lacombe, C., Mangeney, A., Mitchell, J., Schwartz, S. J. and Robert, P. 2009 Universality of solar-wind turbulent spectrum from MHD to electron scales. Phys. Rev. Lett. 103(16), 165003.

Andronov, A. A. and Trakhtengerts, V. Y. 1964 Kinetic instability of the Earth's outer radiation belt. Geomagnetism and Aeronomy 4, 233-242.

Angelopoulos, V., Baumjohann, W., Kennel, C. F., Coroniti, F. V., Kivelson, M. G., Pellat, R., Walker, R. J., Lühr, H. and Paschmann, G. 1992 Bursty bulk flows in the inner central plasma sheet. J. Geophys. Res. 97, 4027-4039.

Angelopoulos, V., Mozer, F. S., Mukai, T., Tsuruda, K., Kokubun, S. and Hughes, T. J. 1999 On the relationship between bursty flows, current disruption and substorms. Geophys. Res. Lett. 26, 2841.

Antiochos, S. K., Karpen, J. T. and DeVore, C. R. 2002 Coronal magnetic field relaxation by null-point reconnection. Astrophys. J. 575(1), 578-584.

Ashour-Abdalla, M., El-Alaoui, M., Goldstein, M. L., Zhou, M., Schriver, D., Richard, R., Walker, R., Kivelson, M. G. and Hwang, K.-J. 2011 Observations and simulations of non-local acceleration of electrons in magnetotail magnetic reconnection events, nature physics. Nature Phys. 7, 360-365.

Ashour-Abdalla, M. and Kennel, C. F. 1978 Nonconvective and convective electron cyclotron harmonic instabilities. J. Geophys. Res. 83, 1531.

Auer, P. L., Kilb, R. W. and Crevier, W. F. 1971 Thermalization in the Earth's bow shock. J. Geophys. Res. 76, 2927.

Bale, S. D., Kasper, J. C., Howes, G. G., Quataert, E., Salem, C. and Sundkvist, D. 2009 Magnetic fluctuation power near proton temperature anisotropy instability thresholds in the solar wind. Phys. Rev. Lett. 103, 211101.

Bale, S. D., Kellogg, P. J., Mozer, F. S., Horbury, T. S. and Rème, H. 2005 Measurement of the electric fluctuation spectrum of magnetohydrodynamic turbulence. Phys. Rev. Lett. 94, 215002.

Balogh, A. et al. 1997 The cluster magnetic field investigation. Space Sci. Rev. 79, 65.

Balogh, A. C. et al. 2001 The cluster magnetic field investigation: overview of in-flight performance and initial results. Ann. Geophys. 19, 1207.

Batchelor, G. K. 1953 The Theory of Homogeneous Turbulence. Cambridge Monographs on Mechanics and Applied Mathematics, Cambridge: Cambridge University Press.

Behlke, R., André, M., Bale, S. D., Pickett, J. S., Cattell, C. A., Lucek, E. A. and Balogh, A. 2004 Solitary structures associated with short large-amplitude magnetic structures (SLAMS) upstream of the Earth's quasi-parallel bow shock. Geophys. Res. Lett. 31, 16805.

Bell, T. F., Inan, U. S., Haque, N. and Pickett, J. S. 2009 Source regions of banded chorus. Geophys. Res. Lett. 36, 11101. 
Belmabrouk, H. and Michard, M. 1998 Taylor length scale measurement by laser Doppler velocimetry. Exp. Fluids 25, 69-76.

Benson, R. F. and Calvert, W. 1979 Isis 1 observations at the source of auroral kilometric radiation. Geophys. Res. Lett. 6, 479-482.

Bieber, J. W., Matthaeus, W. H., Smith, C. W., Wanner, W., Kallenrode, M.-B. and Wibberenz, G. 1994 Proton and electron mean free paths: the Palmer consensus revisited. Astrophys. J. 420, 294-306.

Bieber, J. W., Wanner, W. and Matthaeus, W. H. 1996 Dominant two-dimensional solar wind turbulence with implications for cosmic ray transport. J. Geophys. Res. 101, 2511-2422.

Birn, J., Hesse, M., Nakamura, R. and Zaharia, S. 2013 Particle acceleration in dipolarization events. J. Geophys. Res. 118, 1960-1971.

Birn, J., Raeder, R. A., Yang, Y. L., Wolf, R. A. and Hesse, M. 2004 On the propagation of bubbles in the geomagnetic tail. Ann. Geophys. 22, 1773-1786.

Boardsen, S. A., Sundberg, T., Slavin, J. A., Anderson, B. J., Korth, H., Solomon, S. C. and Blomberg, L. G. 2010 Observations of Kelvin-Helmholtz waves along the dusk-side boundary of Mercury's magnetosphere during MESSENGER's third flyby. Geophys. Res. Lett. 37, 12101.

Bogdanov, S. Y., Burilina, V. B., Markov, V. S. and Frank, A. G. 1994 Formation of current sheets in 3d magnetic-fields with a null point. Jetp Lett. 59(8), 537-541.

Borovsky, J. E. 2006 Eddy viscosity and flow properties of the solar wind: co-rotating interaction regions, coronal-mass-ejection sheaths, and solar-wind/magnetosphere coupling. Phys. Plasmas 13(5), 056505.

Bortnik, J., Thorne, R. M. and Meredith, N. P. 2008 The unexpected origin of plasmaspheric hiss from discrete chorus emissions. Nature 452, 62-66.

Bortnik, J., Thorne, R. M. and Meredith, N. P. 2009 Plasmaspheric hiss overview and relation to chorus. J. Atmos. Sol.-Terr. Phys. 71, 1636-1646.

Breneman, A., Kletzing, C. A., Chum, J., Santolik, O., Gurnett, D. A. and Pickett, J. S. 2007 Multispacecraft observations of chorus dispersion and source location. J. Geophys. Res. 112, 5221.

Breneman, A. W., Kletzing, C. A., Pickett, J., Chum, J. and Santolik, O. 2009 Statistics of multispacecraft observations of chorus dispersion and source location. J. Geophys. Res. (Space Phys.) 114, 6202.

Bruno, R. and Carbone, V. 2005 The solar wind as a turbulence laboratory. J. Living Rev. Sol. Phys. 2.

Bruno, R., Carbone, V., Veltri, P., Pietropaolo, E. and Bavassano, B. 2001 Identifying intermittency events in the solar wind. Planet. Space Sci. 49, 1201.

Burgess, D. 1989 On the effect of a tangential discontinuity on ions specularly reflected at an oblique shock. J. Geophys. Res. 94, 472-478.

Burgess, D. 2006 Interpreting multipoint observations of substructure at the quasi-perpendicular bow shock: simulations. J. Geophys. Res. (Space Phys.) 111(A10), 10210.

Burgess, D. et al. 2005 Quasi-parallel shock structure and processes. Space Sci. Rev. 118, 205-222.

Burlaga, L. F. 1968 Micro-scale structures in the interplanetary medium. Sol. Phys. 4, 67.

Burlaga, L. F. 1969 Directional discontinuities in the interplanetary magnetic field. Sol. Phys. 7, 54.

Calvert, W. 1981 The AKR emission cone at low frequencies. Geophys. Res. Lett. 8, 1159-1162.

Camporeale, E. and Burgess, D. 2011a Erratum: 'The dissipation of solar wind turbulent fluctuations at electron scales' (2011, ApJ, 730, 114). Astrophys. J. 735, 67.

Camporeale, E. and Burgess, D. 2011b The dissipation of solar wind turbulent fluctuations at electron scales. Astrophys. J. 730, 114.

Carbone, V., Veltri, P. and Mangeney, A. 1990 Coherent structure formation and magnetic field line reconnection in magnetohydrodynamic turbulence. Phys. Fluids A 2, 1487.

Cattell, C. et al. 2005 Cluster observations of electron holes in association with magnetotail reconnection and comparison to simulations. J. Geophys. Res. (Space Phys.) 110, 1211.

Chang, O., Peter Gary, S. and Wang, J. 2011 Whistler turbulence forward cascade: three-dimensional particle-in-cell simulations. Geophys. Res. Lett. 38, 22102.

Chanteur, G. 1998 Spatial interpolation for four spacecraft: theory. ISSI Sci. Rep. Ser. 1, 349-370.

Chapman, S. C., Lee, R. E. and Dendy, R. O. 2005 Perpendicular shock reformation and ion acceleration. Space Sci. Rev. 121, 5-19. 
Chaston, C. C., Wilber, M., Mozer, F. S., Fujimoto, M., Goldstein, M. L., Acuña, M., Rème, H. and Fazakerley, A. 2007 Mode conversion and anomalous transport in Kelvin-Helmholtz vortices and kinetic Alfvén waves at the Earth's magnetopause. Phys. Rev. Lett. 99, 175004.

Chen, C. H. K., Bale, S. D., Salem, C. S. and Maruca, B. A. 2013 Residual energy spectrum of solar wind turbulence. Astrophys. J. 770, 125.

Chen, L.-J. et al. 2009 Multi-spacecraft observations of the electron current sheet, neighboring magnetic islands, and electron acceleration during magnetotail reconnection. Phys. Plasma 16, 056501 .

Chen, L.-J. et al. 2008 Evidence of an extended electron current sheet and its neighboring magnetic island during magnetotail reconnection. J. Geophys. Res. (Space Phys.) 113(A12), 12213.

Chen, L.-J. and Parks, G. K. 2002a Bgk electron solitary waves: 1d and 3d. Nonlinear Proc. Geophys. 9, 111.

Chen, L.-J. and Parks, G. K. 2002b BGK electron solitary waves in 3D magnetized plasma. Geophys. Res. Lett. 29, 1331.

Chum, J., Santolík, O., Breneman, A. W., Kletzing, C. A., Gurnett, D. A. and Pickett, J. S. 2007 Chorus source properties that produce time shifts and frequency range differences observed on different Cluster spacecraft. J. Geophys. Res. (Space Phys.) 112, 6206.

Chum, J., Santolik, O., Gurnett, D. A. and Pickett, J. S. 2009 Oblique lower band chorus waves: time shifts between discrete elements observed by the Cluster spacecraft. J. Geophys. Res. (Space Phys.) 114, A00 F02.

Coates, A. J., Mazelle, C. and Neubauer, F. M. 1997 Bow shock analysis at comets halley and grigg-skjellerup. J. Geophys. Res. 102(A4), 7105-7114.

Constantinescu, O. D., Glassmeier, K.-H., Motschmann, U., Treumann, R. A., Fornaçon, K.-H. and Fränz, M. 2006 Plasma wave source location using CLUSTER as a spherical wave telescope. J. Geophys. Res. (Space Phys.) 111, 9221.

Cornilleau-Wehrlin, N. et al. 2003 First results obtained by the Cluster STAFF experiment. Ann. Geophys. 21, 437.

Coroniti, F. V. 1985 Space plasma turbulent dissipation: reality or Myth? Space Sci. Rev. 42, 399.

Cowee, M. M., Winske, D. and Gary, S. P. 2009 Two-dimensional hybrid simulations of superdiffusion at the magnetopause driven by Kelvin-Helmholtz instability. J. Geophys. Res. 11410209.

Cutler, J. C., Dougherty, M. K., Lucek, E. and Masters, A. 2011 Evidence of surface wave on the dusk flank of Saturn's magnetopause possibly caused by the Kelvin-Helmholtz instability. $J$. Geophys. Res. (Space Phys.) 116(A15), 10220.

Dasso, S., Milano, L. J., Matthaeus, W. H. and Smith, C. W. 2005 Anisotropy in fast and slow solar wind fluctuations. Astrophys. J. 635, L181.

Daughton, W., Lapenta, G. and Ricci, P. 2004 Nonlinear evolution of the lower-hybrid drift instability in a current sheet. Phys. Rev. Lett. 93, 105004.

Décréau, P. M. E. et al. and Group, Whisper Experimenters 2001 Early results from the whisper instrument on cluster: an overview. Ann. Geophys. 19(1), 1241-1258.

Delcourt, D. C. 2002 Particle acceleration by inductive electric fields in the inner magnetosphere. $J$. Atmos. Sol. Terr. Phys. 64, 551-559.

Delcourt, D. C., Pedersen, A. and Sauvaud, J. A. 1990 Dynamics of single-particle orbits during substorm expansion phase. J. Geophys. Res. 95, 20 853-20865.

Delory, G. T., Ergun, R. E., Carlson, C. W., Muschietti, L., Chaston, C. C., Peria, W., McFadden, J. P. and Strangeway, R. 1998 FAST observations of electron distributions within AKR source regions. Geophys. Res. Lett. 25, 2069-2072.

Deng, X., Ashour-Abdalla, M., Zhou, M., Walker, R., El-Alaoui, M., Angelopoulos, V., Ergun, R. E. and Schriver, D. 2010a Correction to 'Wave and particle characteristics of earthward electron injections associated with dipolarization fronts'. J. Geophys. Res. (Space Phys.) 115(A14), 12210.

Deng, X., Ashour-Abdalla, M., Zhou, M., Walker, R., El-Alaoui, M., Angelopoulos, V., Ergun, R. E. and Schriver, D. 2010b Wave and particle characteristics of earthward electron injections associated with dipolarization fronts. J. Geophys. Res. (Space Phys.) 115, 9225.

Deng, X. H. et al. 2006 Observations of electrostatic solitary waves associated with reconnection by Geotail and Cluster. Adv. Space Res. 37, 1373-1381. 
Deng, X. H. et al. 2009 Dynamics and waves near multiple magnetic null points in reconnection diffusion region. J. Geophys. Res. (Space Phys.) 114, 7216.

Dorelli, J. C., Bhattacharjee, A. and Raeder, J. 2007a Correction to 'Separator reconnection at Earth's dayside magnetopause under generic northward interplanetary magnetic field conditions'. $J$. Geophys. Res. (Space Phys.) 112, 6204.

Dorelli, J. C., Bhattacharjee, A. and Raeder, J. 2007b Separator reconnection at Earth's dayside magnetopause under generic northward interplanetary magnetic field conditions. J. Geophys. Res. (Space Phys.) 112, 2202.

Dubyagin, S., Sergeev, V., Apatenkov, S., Angelopoulos, V., Nakamura, R., McFadden, J., Larson, D. and Bonnell, J. 2010 Pressure and entropy changes in the flow-braking region during magnetic field dipolarization. J. Geophys. Res. (Space Phys.) 115(A14), 10225.

Dungey, J. W. 1954 Electrodynamics of the outer atmosphere. In: Physics of the Ionosphere: Report of the Conference Held at the Cavendish Laboratory, Cambridge, England, September, pp. 229, Phys. Soc. London.

Dunlop, M. W., Balogh, A., Glassmeier, K. H. and Robert, P. 2002 Four-point cluster application of magnetic field analysis tools: the curlometer. J. Geophys. Res. (Space Phys.) 107(A11), 1385.

Dunlop, M. W. and Eastwood, J. P. 2008 The curlometer and other gradient based methods. In: Multi-Spacecraft Analysis Methods Revisited / Gotz Paschmann and Patrick W. Daly (eds.). ISSI Scientific Reports Series, Vol. 8, 17-26.

Dunlop, M. W.et al. 2011 Extended magnetic reconnection across the dayside magnetopause. Phys. Rev. Lett. 107, 025004.

Eastwood, J. P., Phan, T. D., Bale, S. D. and Tjulin, A. 2009 Observations of turbulence generated by magnetic reconnection. Phys. Rev. Lett. 102(3), 35001.

Eastwood, J. P., Phan, T. D., Øieroset, M. and Shay, M. A. 2010 Average properties of the magnetic reconnection ion diffusion region in the Earth's magnetotail: the 2001-2005 Cluster observations and comparison with simulations. J. Geophys. Res. (Space Phys.) 115, 8215.

Egedal, J., Fox, W., Porkolab, M., Øieroset, M., Lin, R. P., Daughton, W. and Drake, J. F. 2008 Evidence and theory for trapped electrons in guide field magnetotail reconnection. J. Geophys. Res. 113, 12207.

Egedal, J., Lê, A., Chen, L. J., Lefebvre, B., Daughton, W. and Fazakerley, A. 2010 Cluster observation of bidirectional beams caused by electron trapping durring antiparallel reconnection. $J$. Geophys. Res. 115, 3214.

Egedal, J., Øieroset, M., Fox, W. and Lin, R. P. 2005 In situ discovery of an electrostatic potential, trapping electrons and mediating fast reconnection in the Earth's magnetotail. Phys. Rev. Lett. 94, 25006.

El-Alaoui, M., Ashour-Abdalla, M., Richard, R. L., Goldstein, M., Weygand, J. M. and Walker, R. J. 2010 Global magnetohydrodynamic simulation of reconnection and turbulence in the plasma sheet. J. Geophys. Res. 115, 15653.

Ellacott, S. W. and Wilkinson, W. P. 2003 Heating of directly transmitted ions at low Mach number perpendicular shocks: new insights from a statistical physics formulation. J. Geophys. Res. (Space Phys.) 108, 1409.

Escoubet, C. P., Fehringer, M. and Goldstein, M. L. 2001 The Cluster mission. Ann. Geophys. 19, 1197.

Escoubet, C. P., Laakso, H. and Goldstein, M. 2005 3D Plasma structures observed by the four cluster spacecraft. In: Multiscale Coupling of Sun-Earth Processes (ed. A. T. Y. Liu, Y. Kamide and G. Consolini), p. 459, Amsterdam, Netherlands: Elsevier B. V. ISBN 0-444-51881-9.

Escoubet, C. P., Russell, C. T. and Schmidt, R. 1997 The Cluster and PHOENIX Missions. Dordrecht: Kluwer Academic Publishers.

Escoubet, C. P., Taylor, M. G. G. T., Masson, A., Laakso, H., Volpp, J., Hapgood, M. and Goldstein, M. L. 2013 Dynamical processes in space: Cluster results. Ann. Geophys. 31, 1045-1059.

Fairfield, D. H., Baumjohann, W., Paschmann, G., Luehr, H. and Sibeck, D. G. 1990 Upstream pressure variations associated with the bow shock and their effects on the magnetosphere. $J$. Geophys. Res. 78, 3731-3744. 
Fairfield, D. H., Kuznetsova, M. M., Mukai, T., Nagai, T., Gombosi, T. I. and Ridley, A. J. 2007 Waves on the dusk flank boundary layer during very northward interplanetary magnetic field conditions: observations and simulation. J. Geophys. Res. 1128206.

Farrell, W. M., Desch, M. D., Ogilvie, K. W. and Kaiser, M. L. 2003 The role of upper hybrid waves in magnetic reconnection. Geophys. Res. Lett. 30, 2259.

Feldman, W. C., Asbridge, J. R., Bame, S. J., Montgomery, M. D. and Gary, S. P. 1975 Solar wind electrons. J. Geophys. Res. 80, 4181-4196.

Fok, M.-C., Moore, T. E., Brandt, P. C., Delcourt, D. C., Slinker, S. P. and Fedder, J. A. 2006 Impulsive enhancements of oxygen ions during substorms. J. Geophys. Res. 111, 10222.

Foullon, C., Farrugia, C. J., Fazakerley, A. N., Owen, C. J., Gratton, F. T. and Torbert, R. B. 2008 Evolution of Kelvin-Helmholtz activity on the dusk flank magnetopause. J. Geophys. Res. 113 11203.

Foullon, C., Farrugia, C. J., Fazakerley, A. N., Owen, C. J. and Gratton, F. T. 2010 On the multispacecraft determination of periodic surface wave phase speeds and wavelengths. $J$. Geophys. Res. 1159203.

$\mathrm{Fu}, \mathrm{H}$. S. et al. 2013 Dipolarization fronts as a consequence of transient reconnection: in situ evidence. Geophys. Res. Lett. 40, 6023-6027.

Fu, H. S., Khotyaintsev, Y. V., André, M. and Vaivads, A. 2011 Fermi and betatron acceleration of suprathermal electrons behind dipolarization fronts. Geophys. Res. Lett. 38, 16104.

Fu, H. S., Khotyaintsev, Y. V., Vaivads, A., André, M. and Huang, S. Y. 2012 Occurrence rate of earthward-propagating dipolarization fronts. Geophys. Res. Lett. 39, 10101.

Fujimoto, K. and Sydora, R. D. 2008 Whistler waves associated with magnetic reconnection. Geophys. Res. Lett. 36, L19 112.

Fung, S. F. and Viñas, A. F. 1994 Excitation of high frequency electromagnetic waves by energetic electrons with an effective loss cone distribution. J. Geophys. Res. 99, 8671-8686.

Fuselier, S. A., Petrinec, S. M. and Trattner, K. J. 2010 Antiparallel magnetic reconnection rates at the earth's magnetopause. J. Geophys. Res. 11510207.

Galsgaard, K. and Pontin, D. I. 2011a Current accumulation at an asymmetric 3D null point caused by generic shearing motions. Astron. Astrophys. 534, A2.

Galsgaard, K. and Pontin, D. I. 2011b Steady state reconnection at a single 3D magnetic null point. Astron. and Astrophys. 529, A20.

Gary, S. P. 1993 Theory of Space Plasma Microinstabilities. New York, USA: Cambridge University Press.

Gedalin, M. 1997 Ion heating in oblique low-Mach number shocks. Geophys. Res. Lett. 24, 25112514.

Ghosh, S. S., Pickett, J. S., Lakhina, G. S., Winningham, J. D., Lavraud, B. and DéCréAu, P. M. E. 2008 Parametric analysis of positive amplitude electron acoustic solitary waves in a magnetized plasma and its application to boundary layers. J. Geophys. Res. (Space Phys.) 113, 6218.

Glassmeier, K. H. et al. 2001 Cluster as a wave telescope - first results from the fluxgate magnetometer. Ann. Geophys. 19, 1439.

Gnavi, G., Gratton, F. T., Farrugia, C. J. and Bilbao, L. 2006 The KH stability of the supersonic magnetopause flanks modeled by continuous profiles for the transition. In: Plasma and Fusion Science: 16th IAEA Technical Meeting on Research using Small Fusion Devices (ed. J. J. E. Herrera-Velázquez), American Institute of Physics Conference Series, Vol. 875, pp. 296-299.

Goldstein, M. L., Escoubet, C. P. and Laakso, H. 2006 Multiscale, multipoint measurements from Cluster: advancing our scientific understanding of geospace. Adv. Space Res. 38, 21-36.

Gosling, J. T. 1990 Coronal mass ejections and magnetic flux ropes in interplanetary space. In: Physics of Magnetic Flux Ropes, Geophys. Monograph 58. Washington DC, USA: American Geophysical Union, p. 343.

Gosling, J. T., Bame, S., Feldman, W., McComas, D., Phillips, J. and Goldstein, B. 1993 Counterstreaming suprathermal electron events upstream of corotating shocks in the solar wind beyond 2 AU: Ulysses. Geophys. Res. Lett. 20, 2335.

Gosling, J. T. and Robson, A. E. 1985 Ion reflection, gyration, and dissipation at supercritical shocks. Wash. DC Am. Geophys. Union Geophys. Monogr. Ser. 35, 141-152.

Gosling, J. T., Skoug, R. M., McComas, D. J., Phan, T. D. and Skoug, R. M. 2005 Direct evidence for magnetic reconnection in the solar wind near 1 AU. J. Geophys. Res. 1101107. 
Gosling, J. T. and Szabo, A. 2008 Bifurcated current sheets produced by magnetic reconnection in the solar wind. J. Geophys. Res. 113, 10103.

Gratton, F. T., Bender, L., Farrugia, C. J. and Gnavi, G. 2004 Concerning a problem on the Kelvin-Helmholtz stability of the thin magnetopause. J. Geophys. Res. 109, 4211.

Greco, A., Chuychai, P., Matthaeus, W. H., Servidio, S. and Dmitruk, P. 2008 Intermittent mhd structures and classical discontinuities. Geophys. Res. Lett. 35, L19111.

Greco, A., Matthaeus, W. H., Servidio, S., Chuychai, P. and Dmitruk, P. 2009 Statistical analysis of discontinuities in solar wind ace data and comparison with intermittent mhd turbulence. Astrophys. J. 691, L111.

Greco, A. and Perri, S. 2014 Identification of high shears and compressive discontinuities in the inner heliosphere. Astrophys. J. 784, 163.

Green, J. L. and Gallagher, D. L. 1985 The detailed intensity distribution of the AKR emission cone. J. Geophys. Res. 90, 9641-9649.

Green, J. L., Gurnett, D. A. and Shawhan, S. D. 1977 The angular distribution of auroral kilometric radiation. J. Geophys. Res. 82, 1825-1838.

Greene, J. M. 1992 Locating 3-dimensional roots by a bisection method. J. Comput. Phys. 98(2), 194-198.

Greenstadt, E. W., Green, I. M., Inouye, G. T., Colburn, D. S., Binsack, J. H. and Lyon, E. F. 1970 Dual satellite observations of earth's bow shock. III: field determined shock structure. Cosmic Electrodynamics 1, 316-327.

Grison, B, Sahraoui, F, Lavraud, B, Chust, T, Cornilleau-Wehrlin, N, Reme, H, Balogh, A and André, M 2005 Wave particle interactions in the high-altitude polar cusp: a Cluster case study. Ann. Geophys. 23(1), 3699-3713.

Guo, R. L. et al. 2013 Separator reconnection with antiparallel/component features observed in magnetotail plasmas. J. Geophys. Res. (Space Phys.) 118(10), 6116-6126.

Gurgiolo, C., Goldstein, M. L., Viñas, A. F., Matthaeus, W. H. and Fazakerley, A. N. 2011 Observations of electron vorticity in the inner plasma sheet. Ann. Geophys. 29, 1517-1527.

Gurgiolo, C., Goldstein, M. L., Matthaeus, W. H., Viñas, A. F. and Fazakerley, A. N. 2013 Characteristics of the Taylor microscale in the solar wind/foreshock: magnetic field and electron velocity measurements. Ann. Geophys. 31, 2063-2075.

Gurgiolo, C., Goldstein, M. L., Viñas, A. F. and Fazakerley, A. N. 2010 First measurements of electron vorticity in the foreshock and solar wind. Ann. Geophys. 28, 2187-2200.

Gurnett, D. and Anderson, R. 1981 The kilometric radio emission spectrum: relationship to auroral acceleration processes, in Physics of Auroral Arc Formation. Geophys. Monogr. 25, 341.

Gurnett, D. A. 1985 Plasma waves and instabilities. Wash. DC Am. Geophys. Union Geophys. Monogr. Ser. 35, 207-224.

Gurnett, D. A., Anderson, R. R., Scarf, F. L., Fredricks, R. W. and Smith, E. J. 1979 Initial results from the ISEE-1 and -2 plasma wave investigation. Space Sci. Rev. 23, 103-122.

Gurnett, D. A. et al. 2001 First results from the Cluster wideband plasma wave investigation. Ann. Geophys. 19(1), 1259-1272.

Gustafsson, G. et al. 2001 First results of electric field and density observations by Cluster EFW based on initial months of operation. Ann. Geophys. 19(1), 1219-1240.

Hada, T., Koga, D. and Yamamoto, E. 2003 Phase coherence of mhd waves in the solar wind. Space Sci. Rev. 107, 463.

Haque, N., Inan, U. S., Bell, T. F. and Pickett, J. S. 2012 Spatial dependence of banded chorus intensity near the magnetic equator. Geophys. Res. Lett. 39, 17103.

Haque, N., Inan, U. S., Bell, T. F., Pickett, J. S., Trotignon, J. G. and Facskó, G. 2011 Cluster observations of whistler mode ducts and banded chorus. Geophys. Res. Lett. 38, 18107.

Hasegawa, A. 1975 Plasma Instabilities and Non-Linear Effects. New York: Springer-Verlag.

Hasegawa, H., Fujimoto, M., Phan, T. D., Rème, H., Balogh, A., Dunlop, M. W., Hashimoto, C. and TanDokoro, R. 2004 Transport of solar wind into Earth's magnetosphere through rolled-up Kelvin-Helmholtz vortices. Nature 430, 755-758.

Hasegawa, H. et al. 2009 Kelvin-Helmholtz waves at the Earth's magnetopause: multiscale development and associated reconnection. J. Geophys. Res. 114, 12207.

He, J.-S., Marsch, E., Tu, C. Y., Zong, Q. G., Yao, S. and Tian, H. 2011 Two-dimensional correlation functions for density and magnetic field fluctuations in magnetosheath turbulence measured by the Cluster spacecraft. J. Geophys. Res. 116(A6), 6207. 
He, J.-S. et al. 2008a A magnetic null geometry reconstructed from Cluster spacecraft observations. J. Geophys. Res. (Space Phys.) 113, 5205.

He, J.-S. et al. 2008b Electron trapping around a magnetic null. Geophys. Res. Lett. 35, 14104.

Hones, E. W. Jr, Paschmann, G., Bame, S. J., Asbridge, J. R., Sckopke, N. and Schindler, K. 1978 Vortices in magneto spheric plasma flow. Geophys. Res. 5(12), 1059.

Horbury, T. S., Cargill, P. J., Lucek, E. A., Eastwood, J., Balogh, A., Dunlop, M. W., Fornacon, K.-H. and Georgescu, E. 2002 Four spacecraft measurements of the quasiperpendicular terrestrial bow shock: orientation and motion. J. Geophys. Res. (Space Phys.) 107, 1208.

Horne, R. B. et al. 2005 Wave acceleration of electrons in the van allen radiation belts. Nature 8(doi:10. 1038/nature03939), 437.

Hoshino, M. 2005 Electron surfing acceleration in magnetic reconnection. J. Geophys. Res. 110, 10215.

Hoshino, M., Mukai, T., Terasawa, T. and Shinohara, I. 2001 Superthermal electron acceleration in magnetic reconnection. J. Geophys. Res. 106, 25972.

Howes, G. G., TenBarge, J. M., Dorland, W., Quataert, E., Schekochihin, A. A., Numata, R. and Tatsuno, T. 2011 Gyrokinetic simulations of solar wind turbulence from ion to electron scales. Phys. Rev. Lett. 107(3), 35004.

Huang, S. Y., Sahraoui, F., Deng, X. H., He, J. S., Yuan, Z. G., Zhou, M., Pang, Y. and Fu, H. S. 2014 Kinetic turbulence in the terrestrial magnetosheath: Cluster observations. Astrophys. J. Lett. 789, L28.

Huang, S. Y., Zhou, M., Deng, X. H., Yuan, Z. G., Pang, Y., Wei, Q., Su, W., Li, H. M. and Wang, Q. Q. 2012 Kinetic structure and wave properties associated with sharp dipolarization front observed by Cluster. Ann. Geophys. 30, 97-107.

Huang, S. Y., Zhou, M., Sahraoui, F., Deng, X.-H., Pang, Y., Yuan, Z. G., Wei, Q., Wang, J. F. and Zhou, X. M. 2010 Wave properties in the magnetic reconnection diffusion region with high $\beta$ : application of the k-filtering method to Cluster multispacecraft data. J. Geophys. Res. (Space Phys.) 115(A), 12211.

Hwang, K.-J., Goldstein, M. L., Kuznetsova, M. M., Wang, Y., Viñas, A. F. and Sibeck, D. G. 2012 The first in situ observation of Kelvin-Helmholtz waves at high-latitude magnetopause during strongly dawnward interplanetary magnetic field conditions. J. Geophys. Res. (Space Phys.) 117, 8233.

Hwang, K.-J., Goldstein, M. L., Lee, E. and Pickett, J. S. 2011a Cluster observations of multiple dipolarization fronts. J. Geophys. Res. (Space Phys.) 116, A00I32.

Hwang, K.-J., Goldstein, M. L., Moore, T. E., Walsh, B. M., Baishev, D. G., Moiseyev, A. V., Shevtsov, B. M. and Yumoto, K. 2014b A tailward moving current sheet normal magnetic field front followed by an earthward moving dipolarization front. J. Geophys. Res. 119, 5316.

Hwang, K.-J., Goldstein, M. L., Viñas, A. F., Schriver, D. and Ashour-Abdalla, M. 2014a Waveparticle interactions during a dipolarization event. J. Geophys. Res. 119, 2484.

Hwang, K.-J., Goldstein, M. L., Wendel, D. E., Fazakerley, A. N. and Gurgiolo, C. 2013 Cluster observations near reconnection x lines in Earth's magnetotail current sheet. J. Geophys. Res. 118, 4199.

Hwang, K.-J., Kuznetsova, M. M., Sahraoui, F., Goldstein, M. L., Lee, E. and Parks, G. K. 2011b Kelvin-Helmholtz waves under southward interplanetary magnetic field. J. Geophys. Res. (Space Phys.) 116, 8210.

Inan, U. S., Platino, M., Bell, T. F., Gurnett, D. A. and Pickett, J. S. 2004 Cluster measurements of rapidly moving sources of ELF/VLF chorus. J. Geophys. Res 109, 5214.

Johnstone, A. D. et al. 1997 PEACE: a plasma electron and current experiment. Space Sci. Rev. 79, 351-398.

Jokipii, J. R. and Hollweg, J. V. 1970 Interplanetary scintillations and the structure of solar-wind fluctuations. Astrophys. J. 160, 745.

Jones, S. T., Fok, M. and Brandt, P. C. 2006 Modeling global $\mathrm{O}^{+}$substorm injection using analytic magnetic field model. J. Geophys. Res. 111, $11 \mathrm{~S} 07$.

Kailasnath, P. and Sreenivasan, K. R. 1993 Zero crossings of velocity fluctuations in turbulent boundary layers. Phys. Fluids A 11, 2879-2885.

Karimabadi, H., Daughton, W. and Scudder, J. 2007 Multi-scale structure of the electron diffusion region. Geophys. Res. Lett. 34, 13104. 
Kaufmann, R. L. 1967 Shock observations with the Explorer 12 magnetometer. J. Geophys. Res. 72, 2323-2342.

Keika, K., Brandt, P. C., Ohtani, S., Mitchell, D. G., Min, K., Nosé, M., Obara, T., Koshiishi, H. and Matsumoto, H. 2010 Mass-dependent evolution of energetic neutral atoms energy spectra during storm time substorms: implication for $\mathrm{O}^{+}$nonadiabatic acceleration. J. Geophys. Res. (Space Phys.) 115, A00 I12.

Keith, W. R., Winningham, J. D., Goldstein, M. L., Wilber, M., Fazakerley, A. N., Rème, H., Fritz, T. A., Balogh, A., Cornilleau-Wehrlin, N. and Maksimovic, M. 2005 Observations of a unique cusp signature at low and mid altitudes. Surv. Geophys. 26, 307-339.

Kennel, C. F., Edmiston, J. P. and Hada, T. 1985 A quarter century of collisionless shock research. Wash. DC Am. Geophys. Union Geophys. Monogr. Ser. 34, 1-36.

Kennel, C. F. and Petschek, H. E. 1966 Limit on stable trapped particle fluxes. J. Geophys. Res. 71, $1-28$.

Kintner, P. M. and Seyler, C. E. 1985 The status of observations and theory of high latitude ionospheric and magnetospheric plasma turbulence. Space Sci. Rev. 41, 94-129.

Kiyani, K., Chapman, S., Khotyaintsev, Y., Dunlop, M. and Sahraoui, F. 2009 Global scale-invariant dissipation in collisionless plasma turbulence. Phys. Rev. Lett. 103, 075006.

Kiyani, K. H., Chapman, S. C., Sahraoui, F., Hnat, B., Fauvarque, O. and Khotyaintsev, Yu V. 2013 Enhanced magnetic compressibility and isotropic scale invariance at sub-ion Larmor scales in solar wind turbulence. Astrophys. J. 763(1), 10.

Koga, D., Chian, A. C.-L., Miranda, R. A. and Rempel, E. L. 2007 Intermittent nature of solar wind turbulence near the earth's bow shock: phase coherence and non-gaussianity. Phys. Rev. E 75, 046401.

Kozelov, B., Demekhov, A. G., Titova, E. E., Trakhtengerts, V. Y., Santolik, O., Macusova, E., Gurnett, D. A. and Pickett, J. S. 2008 Variations in the chorus source location deduced from fluctuations of the ambient magnetic field: comparison of cluster data and the backward-wave oscillator model. J. Geophys. Res. 113, 6216.

Krall, N. A. 1997 What do we really know about collisionless shocks? Adv. Space Res. 20, 715-724.

Kuznetsova, M., Sibeck, D. G., Hesse, M., Rastaetter, L., Toth, G. and Gombosi, T. 2008 Global MHD simulations of dayside magnetopause dynamics. AGU Fall 2008 Meeting.

Laakso, H., Taylor, M. and Escoubet, C. P. 2010 The Cluster Active Archive. Springer, Dordrecht.

Lacombe, C., Samsonov, A. A., Mangeney, A., Maksimovic, M., Cornilleau-Wehrlin, N., Harvey, C. C., Bosqued, J. M. and Travnicek, P. 2006 Cluster observations in the magnetosheath Part 2: intensity of the turbulence at electron scales. Ann. Geophys. 24(1), 3523-3531.

Lakhina, G. S., Singh, S. V., Kakad, A. P., Goldstein, M. L., Viñas, A. F. and Pickett, J. S. 2009 A mechanism for electrostatic solitary structures in the Earth's magnetosheath. J. Geophys. Res. (Space Phys.) 114, 9212.

Lakhina, G. S., Singh, S. V., Kakad, A. P. and Pickett, J. S. 2011 Generation of electrostatic solitary waves in the plasma sheet boundary layer. J. Geophys. Res. (Space Phys.) 116(A15), 10218.

Lakhina, G. S., Tsurutani, B. T., Verkhoglyadova, O. P. and Pickett, J. S. 2010 Pitch angle transport of electrons due to cyclotron interactions with the coherent chorus subelements. J. Geophys. Res. (Space Phys.) 115, A00 F15.

Le, A., Egedal, J., Daughton, W., Drake, J. F., Fox, W. and Katz, N. 2010 Magnitude of the hall fields during magnetic reconnection. Geophys. Res. Lett. 373106.

Le, A., Egedal, J., Daughton, W., Fox, W. and Katz, N. 2009 Equations of state for collisionless guide-field reconnection. Phys. Rev. Lett. 102, 085001.

Le, A., Egedal, J., Daughton, W., Karimabadi, H., Ohia, O. and Lukin, V. S. 2013 Regimes of the electron diffusion region in magnetic reconnection. Phys. Rev. Lett. 110, 135004.

Lee, S. H., Zhang, H., Zong, Q.-G., Otto, A., Sibeck, D. G., Wang, Y., Glassmeier, K.-H., Daly, P. W. and Réme, H. 2013 Plasma and energetic particle behaviors during asymmetric magnetic reconnection at the magnetopause. J. Geophys. Res. 119, 1658-1672.

Lefebvre, B., Chen, L.-J., Gekelman, W., Kintner, P., Pickett, J., Pribyl, P. and Vincena, S. 2011 Debye-scale solitary structures measured in a beam-plasma laboratory experiment. Nonlinear Process. Geophys. 18, 41-47.

Lembège, B. and Savoini, P. 2002 Formation of reflected electron bursts by the nonstationarity and nonuniformity of a collisionless shock front. J. Geophys. Res. (Space Phys.) 107, 1037. 
Leroy, M. M., Goodrich, C. C., Winske, D., Wu, C. S. and Papadopoulos, K. 1981 Simulation of a perpendicular bow shock. Geophys. Res. Lett. 8, 1269-1272.

Li, S.-Y., Deng, X.-H., Zhou, M., Yuan, Z.-G., Wang, J.-F., Lin, X., Lin, M.-H. and Fu, S. 2010 Geophysics, astronomy, and astrophysics: Cluster observation of electrostatic solitary waves around magnetic null point in thin current sheet. Chinese Phys. Lett. 27(1), 019401.

Lin, R. P. et al. 1997 Observations of the solar wind, bow shock and upstream particles with the WIND 3D plasma instrument. Adv. Space Res. 20, 645-654.

Lin, Y. and Lee, L. C. 1994 Reconnection layer at the flank magnetopause in the presence of shear flow. Geophys. Res. Lett. 21, 855-858.

Lockwood, M. and Smith, F. M. 1992 The variation of reconnection rate at the dayside magnetopause and cusp ion precipitation. JGR 97, 14841-14847.

Lucek, E. A., Dunlop, M. W., Horbury, T. S., Balogh, A., Brown, P., Cargill, P., Carr, C., Fornacon, K. H., Georgescu, E. and Oddy, T. 2001 Cluster magnetic field observations in the magnetosheath: four-point measurements of mirror structures. Ann. Geophys. 19, 1421-1428.

Lucek, E. A., Horbury, T. S., Dandouras, I. and RèMe, H. 2008 Cluster observations of the Earth's quasi-parallel bow shock. J. Geophys. Res. (Space Phys.) 113, 7.

Lukin, V. S. and Linton, M. G. 2011 Three-dimensional magnetic reconnection through a moving magnetic null. Nonlinear Process. Geophys. 18(6), 871-882.

Maksimovic, M. V., Pierrard, V. and Riley, P. 1997 Ulysses electron distributions fitted with kappa functions. Geophys. Res. Lett. 24, 1151.

Maksimovic, M. V. et al. 2005 Radial evolution of the electron distribution functions in the fast solar wind between 0.3 and 1.5 AU. J. Geophys. Res. 110, 9104.

Mangeney, A., Lacombe, C., Maksimovic, M., Samsonov, A. A., Cornilleau-Wehrlin, N., Harvey, C. C., Bosqued, J.-M. and Trávníček, P. 2006 Cluster observations in the magnetosheath Part 1: anisotropies of the wave vector distribution of the turbulence at electron scales. Ann. Geophys. 24, 3507.

Marsch, E. and Tu, C. Y. 1997 Intermittency, non-gaussian statistics and fractal scaling of mhd fluctuations in the solar wind. Nonlinear Proc. Geophys. 4, 101.

Masters, A. et al. 2010 Cassini observations of a Kelvin-Helmholtz vortex in Saturn's outer magnetosphere. J. Geophys. Res. 115, 7225.

Matsumoto, Y. and Hoshino, M. 2004 Onset of turbulence induced by a Kelvin-Helmholtz vortex. Geophys. Res. Lett. 312807.

Matsumoto, Y. and Hoshino, M. 2006 Turbulent mixing and transport of collisionless plasmas across a stratified velocity shear layer. J. Geophys. Res. 1115213.

Matthaeus, W. H. 1982 Reconnection in two dimensions: localization of vorticity and current near the x-points. Geophys. Res. Lett. 9, 660-663.

Matthaeus, W. H., Dasso, S., Weygand, J. M., Milano, L. J., Smith, C. W. and Kivelson, M. J. 2005 Spatial correlation of the solar wind turbulence from two point measurements. Phys. Rev. Lett. 95, 231 101-231 104.

Matthaeus, W. H., Goldstein, M. L. and King, J. H. 1986a An interplanetary field ensemble at 1 AU. J. Geophys. Res. 91, 59.

Matthaeus, W. H., Goldstein, M. L. and Lantz, S. R. 1986b The alpha dynamo parameter and measurability of helicities in magnetohydrodynamic turbulence. Phys. Fluids 29, 1504.

Matthaeus, W. H., Goldstein, M. L. and Roberts, D. A. 1990 Evidence for the presence of quasitwo-dimensional, nearly incompressible fluctuations in the solar wind. J. Geophys. Res. 95, 20673.

Matthaeus, W. H. and Lamkin, L. 1985 Rapid magnetic reconnection caused by finite amplitude fluctuations. Phys. Fluids 28, 303.

Matthaeus, W. H. and Montgomery, D. 1980 Selective decay hypothesis at high mechanical and magnetic Reynolds numbers. Ann. New York Acad. Sci. 357, 203-222.

Menietti, J. D., Mutel, R. L., Santolik, O., Scudder, J. D., Christopher, I. W. and Cook, J. M. 2006 Striated drifting auroral kilometric radiation bursts: possible stimulation by upward traveling EMIC waves. J. Geophys. Res. (Space Phys.) 111, 4214.

Menietti, J. D., Persoon, A. M., Pickett, J. S. and Gurnett, D. A. 2000 Statistical study of auroral kilometric radiation fine structure striations observed by Polar. J. Geophys. Res. (Space Phys.) 105, 18857. 
Menietti, J. D., Wong, H. K., Kurth, W. S., Gurnett, D. A., Granroth, L. J. and Groene, J. B. 1996 Discrete, stimulated auroral kilometric radiation observed in the Galileo and DE 1 wideband data. J. Geophys. Res. (Space Phys.) 101, 10673-10680.

Meyrand, R. and Galtier, S. 2010 A universal law for solar-wind turbulence at electron scales. Astrophys. J. 721(2), 1421-1424.

Meyrand, R. and Galtier, S. 2012 Spontaneous chiral symmetry breaking of hall magnetohydrodynamic turbulence. Phys. Rev. Lett. 109(1), 194501.

Miura, A. 1995 Dependence of the magnetopause Kelvin-Helmholtz instability on the orientation of the magnetosheath magnetic field. Geophys. Res. Lett. 22, 2993.

Miura, A. and Pritchett, P. L. 1982 Nonlocal stability analysis of the MHD Kelvin-Helmholtz instability in a compressible plasma. J. Geophys. Res. 87, 7431.

Mozer, F. S., Bale, S. D., McFadden, J. P. and Torbert, R. B. 2005 New features of electron diffusion regions observed at subsolar magnetic field reconnection sites. Geophys. Res. Lett. 32, 24102.

Mutel, R., Gurnett, D. and Christopher, I. 2004 Spatial and temporal properties of AKR burst emission derived from Cluster WBD VLBI studies. Ann. Geophys. 22, 2625-2632.

Mutel, R. L., Christopher, I. W. and Pickett, J. S. 2008 Cluster multispacecraft determination of AKR angular beaming. Geophys. Res. Lett. 35, 7104.

Mutel, R. L., Gurnett, D. A., Christopher, I. W., Pickett, J. S. and Schlax, M. 2003 Locations of auroral kilometric radiation bursts inferred from multispacecraft wideband Cluster VLBI observations. 1: description of technique and initial results. J. Geophys. Res. (Space Phys.) 108, 1398.

Mutel, R. L., Menietti, J. D., Christopher, I. W., Gurnett, D. A. and Cook, J. M. 2006 Striated auroral kilometric radiation emission: a remote tracer of ion solitary structures. J. Geophys. Res. (Space Phys.) 111(A10), 10203.

Mutel, R. L., Peterson, W. M., Jaeger, T. R. and Scudder, J. D. 2007 Dependence of cyclotron maser instability growth rates on electron velocity distributions and perturbation by solitary waves. J. Geophys. Res. 1127211.

Nakamura, R. et al. 2002 Motion of the dipolarization front during a flow burst event observed by Cluster. Geophys. Res. Lett. 29, 1942.

Nakamura, R., Retinò, A., Baumjohann, W., Volwerk, M., Erkaev, N., Klecker, B., Lucek, E. A., Dandouras, I., André, M. and Khotyaintsev, Y. 2009 Evolution of dipolarization in the near-Earth current sheet induced by Earthward rapid flux transport. Ann. Geophys. 27, 1743.

Nakamura, T. K. M., Fujimoto, M. and Otto, A. 2006 Magnetic reconnection induced by weak Kelvin-Helmholtz instability and the formation of the low-latitude boundary layer. Geophys. Res. Lett. 3314016.

Nakamura, T. K. M., Fujimoto, M. and Otto, A. 2008 Structure of an MHD-scale Kelvin-Helmholtz vortex: two-dimensional two-fluid simulations including finite electron inertial effects. $J$. Geophys. Res. 113, A09 204.

Nakamura, T. K. M., Hayashi, D., Fujimoto, M. and Shinohara, I. 2004 Decay of MHD-scale Kelvin-Helmholtz vortices mediated by parasitic electron dynamics. Phys. Rev. Lett. 92, 145001.

Narita, Y., Glassmeier, K. H., Sahraoui, F., Goldstein, M. L. and Treumann, R. A. 2010 Wave-vector dependence of magnetic-turbulence spectra in the solar wind. Phys. Rev. Lett. 104, 171101.

Narita, Y., Glassmeier, K. H. and Treumann, R. A. 2006 Wave-number spectra and intermittency in the terrestrial foreshock region. Phys. Rev. Lett. 97, 191 101-191 104.

Ness, N. F., Scearce, C. S. and Seek, J. B. 1964 Initial results of the Imp 1 magnetic field experiment. J. Geophys. Res. 69, 3531-3569.

Neubauer, F. and Glassmeier, K-H. 1987 Use of an array of satellites as a wave telescope. $J$. Geophys. Res. 95, 19115-19122.

Nieves-Chinchilla, T. and Viñas, A. F. 2008 Solar wind electron distribution functions inside magnetic clouds. J. Geophys. Res. 113, 2105.

Nunn, D., Omura, Y., Matsumoto, H., Nagano, I. and Yagitani, S. 1997 The numerical simulation of VLF chorus and discrete emissions observed on the Geotail satellite using a Vlasov code. J. Geophys. Res. 102, $27083-27097$.

Nykyri, K., Grison, B., Cargill, P. J., Lucek, E., Dandouras, I., Balogh, A., Cornilleau-Wehrlin, N. and Rème, H. 2006 Origin of the turbulent spectra in the high-alititude cups: Cluster spacecraft observations. Ann. Geophys. 24, 1057. 
Nykyri, K. and Otto, A. 2001 Plasma transport at the magnetospheric boundary due to reconnection in Kelvin-Helmholtz vortices. Geophys. Res. Lett. 28, 3565.

Ofman, L. and Thompson, B. J. 2011 SDO/AIA Oobservation of Kelvin-Helmholtz instability in the solar corona. Astrophys. J. Lett. 734 L11.

Ohia, O., Egedal, J., Lukin, V. S., Daughton, W. and Le, A. 2012 Demonstration of anisotropic fluid closure capturing the kinetic structure of magnetic reconnectionl. Phy. Rev. Lett. 109, 115004

Ohtani, S., Brandt, P. C., Mitchell, D. G., Singer, H., Nosé, M., Reeves, G. D. and Mende, S. B. 2005 Storm-substorm relationship: variations of the hydrogen and oxygen energetic neutral atom intensities during storm-time substorms. J. Geophys. Res. 110, 7219.

Øieroset, M., Lin, R. and Phan, T. D. 2002 Evidence for electron acceleration up to similar to 300 $\mathrm{keV}$ in the magnetic reconnection diffusion region of earth's magnetotail. Phy. Rev. Lett. 89, 195001.

Osman, K. T. and Horbury, T. S. 2007 Multispacecraft measurement of anisotropic correlation functions in solar wind turbulence. Astrophys. J. Lett. 654, L103-L106.

Osman, K. T., Wan, M., Matthaeus, W. H., Breech, B. and Oughton, S. 2011 Directional alignment and non-Gaussian statistics in solar wind turbulence. Astrophys. J. 75, 741.

Otto, A. and Fairfield, D. H. 2000 Kelvin-Helmholtz instability at the magnetotail boundary: MHD simulation and comparison with Geotail observations. J. Geophys. Res. 105, 21175.

Owen, C. J., Taylor, M. G. G. T., Krauklis, I. C., Fazakerley, A. N., Dunlop, M. W. and Bosqued, J. M. 2004 Cluster observations of surface waves on the dawn flank magnetopause. Ann. Geophys. 22, 971-983.

Palermo, F., Faganello, M, Califano, E. and Pegorano, F. 2011 Kelvin-Helmholtz vortices and secondary instabilities in super-magnetosonic regimes. Ann. Geophys. 29, 1169-1178.

Parks, G. K. et al. 2012 Entropy generation across Earth's collisionless bow shock. Phys. Rev. Lett. 108(6), 061102.

Parnell, C. E. 2007 Multiply connected source and null pairs. Sol. Phys. 242(1-2), 21-41.

Parnell, C. E., Haynes, A. L. and Galsgaard, K. 2008 Recursive reconnection and magnetic skeletons. Astrophys. J. 675(2), 1656-1665.

Paschmann, G. 1983 Collisionless shocks. ESA J. 7, 357-368.

Paschmann, G. 2008 Recent in-situ observations of magnetic reconnection in near-Earth space. Geophys. Res. Lett. 35, 19109.

Paschmann, G. and Daly, P. W. 1998 Analysis methods for multi-spacecraft data. ISSI Scientific Reports Series SR-001, ESA/ISSI, Vol. 1. ISBN 1608-280X, 1998. Analysis Methods for MultiSpacecraft Data / Götz Paschmann and Patrick Daly (eds.). ISSI Scientific Reports Series 1. ISSI Scientific Reports Series, ESA/ISSI. ISBN 1608-280X.

Paschmann, G. et al. 2001 The electron drift instrument on Cluster: overview of first results. Ann. Geophys. 19(1), 1273-1288.

Paschmann, G., Schwartz, S. J., Escoubet, C. P. and Haaland, S. 2005 Outer Magnetospheric Boundaries: Cluster Results, Space Sciences Series of ISSI, vol. 118. Berlin: Springer.

Perri, S., Goldstein, M. L., Dorelli, J. C. and Sahraoui, F. 2012 Detection of small-scale structures in the dissipation regime of solar-wind turbulence. Phys. Rev. Lett. 109, 191101.

Perri, S., Yordanova, E., Carbone, V., Veltri, P., Sorriso-Valvo, L., Bruno, R. and André, M. 2009 Magnetic turbulence in space plasmas: scale-dependent effects of anisotropy. J. Geophys. Res. 114, 2102.

Perrone, D., Valentini, F., Servidio, S., Dalena, S., and Veltri, P. 2013 Vlasov simulations of multi-ion plasma turbulence in the solar wind. Astrophys. J. 762, 99.

Pickett, J., Chen, L., Kahler, S., Santolík, O., Gurnett, D., Tsurutani, B. and Balogh, A. 2004a Isolated electrostatic structures observed throughout the Cluster orbit: relationship to magnetic field strength. Ann. Geophys. 22, 2515-2523.

Pickett, J. S. et al. 2005 On the generation of solitary waves observed by Cluster in the near-Earth magnetosheath. Nonlinear Process. Geophys. 12, 181-193.

Pickett, J. S. et al. 2008 Furthering our understanding of electrostatic solitary waves through Cluster multispacecraft observations and theory. Adv. Space Res. 41, 1666-1676.

Pickett, J. S. et al. 2011 On the propagation and modulation of electrostatic solitary waves observed near the magnetopause on Cluster. In: American Institute of Physics Conference Series (ed. D. Vassiliadis, S. F. Fung, X. Shao, I. A. Daglis and J. D. Huba), American Institute of Physics Conference Series, Vol. 1320, pp. 115-124. 
Pickett, J. S. et al. 2004b Solitary waves observed in the auroral zone: the Cluster multi-spacecraft perspective. Nonlinear Process. Geophys. 11, 183-196.

Pilipp, W. G., Miggenrieder, H., Montgomery, M. D., Mühlhaüser, K.-H., Rosenbauer, H. and Schwenn, R. 1987a Characteristics of electron velocity distribution functions in the solar wind derived from Helios Plasma Experiment. J. Geophys. Res. 92, 1075-1092.

Pilipp, W. G., Miggenrieder, H., Montgomery, M. D., Mühlhaüser, K.-H., Rosenbauer, H. and Schwenn, R. 1987b Unusual electron distribution functions in the solar wind derived from the helios plasma experiment: double-strahl distributions and distributions with an extremely anisotropic core. J. Geophys. Res. 92, 1093-1101.

Pilipp, W. G., Miggenrieder, H., Mühlhaüser, K.-H., Rosenbauer, H., Schwenn, R. and Neubauer, F. M. $1987 \mathrm{c}$ Variations of electron velocity distribution functions in the solar wind. J. Geophys. Res. 92, 1103-1118.

Pincon, J. L. and Lefeuvre, F. 1988 Characterization of a homogeneous field turbulence from multipoint measurements. COSPAR and SCOSTEP 8, 459.

Pinçon, J. L. and Lefeuvre, F. 1991 Local characterization of homogeneous turbulence in a space plasma from simultaneous measurements of field components at several points in space. $J$. Geophys. Res. 96, 1789.

Pinçon, J. L. and Lefeuvre, F. 1992 The application of the generalized Capon method to the analysis of a turbulent field in space plasma: experimental constraints. J. Atmos. Terr. Phys. 54, 1237-1247.

Pinçon, J-L. and Motschmann, U. 1998 Multi-spacecraft filtering: general framework. In: Analysis Methods for Multi-Spacecraft Data (ed. G. Paschmann and P. W. Daly), Keplerlaan 1, 2200 AG Noordwijk, The Netherlands: ESA Publications Division, pp. 79-91.

Platino, M., Inan, U. S., Bell, T. F., Pickett, J. S. and Canu, P. 2006 Rapidly moving sources of upper-band ELF/vlf chorus near the magnetic equator. J. Geophys. Res. 111, 09218.

Podesta, J. J., Borovsky, J. E. and Gary, S. P. 2010 A kinetic Alfvén wave cascade subject to collisionless damping cannot reach electron scales in the solar wind at 1 AU. Astrophys. $J$. 712(1), 685-691.

Pontin, D. I., Al-Hachami, A. K. and Galsgaard, K. 2011 Generalised models for torsional spine and fan magnetic reconnection. Astron. Astrophys. 533, 78.

Pontin, D. I., Bhattacharjee, A. and Galsgaard, K. 2007 Current sheet formation and nonideal behavior at three-dimensional magnetic null points. Phys. Plasmas 14(5), 2106.

Pontin, D. I. and Galsgaard, K. 2007 Current amplification and magnetic reconnection at a threedimensional null point: physical characteristics. J. Geophys. Res. (Space Phys.) 112(A3), 3103.

Pontin, D. I., Hornig, G. and Priest, E. R. 2004 Kinematic reconnection at a magnetic null point: spine-aligned current. Geophys. Fluid Dyn. 98(5), 407-428.

Pontin, D. I., Hornig, G. and Priest, E. R. 2005 Kinematic reconnection at a magnetic null point: fan-aligned current. Geophys. Astrophys. Fluid Dyn. 99(1), 77-93.

Pontin, D. I., Priest, E. R. and Galsgaard, K. 2013 On the nature of reconnection at a solar coronal null point above a separatrix dome. Astrophys. J. 774(2) 154.

Pope, S. A., Balikhin, M. A., Zhang, T. L., Fedorov, A. O., Gedalin, M. and Barabash, S. 2009 Giant vortices lead to ion escape from Venus and re-distribution of plasma in the ionosphere. Geophys. Res. Lett. 36, 7202.

Pottelette, R., Treumann, R. A. and Berthomier, M. 2001 Auroral plasma turbulence and the cause of auroral kilometric radiation fine structure. J. Geophys. Res. (Space Phys.) 106, 84658476.

Priest, E. R. and Pontin, D. I. 2009 Three-dimensional null point reconnection regimes. Phys. Plasmas 16, 2101.

Priest, E. R. and Titov, V. S. 1996 Magnetic reconnection at three-dimensional null points. Phil. Trans. R. Soc. Lond. Ser. A-Math. Phys. Eng. Sci. 354(1721), 2951-2992.

Pritchett, P. L., Strangeway, R. J., Ergun, R. E. and Carlson, C. W. 2002 Generation and propagation of cyclotron maser emissions in the finite auroral kilometric radiation source cavity. J. Geophys. Res. (Space Phys.) 107, 1437.

Rankin, D., Frycz, P., Samson, J. C. and Tikhonchuk, V. T. 1997 Shear flow vortices in magnetospheric plasmas. Phys. Plasmas 4, 829.

Reme, H. et al. 2001 First multispacecraft ion measurements in and near the Earth's magnetosphere with the identical Cluster ion spectrometry (CIS) experiment. Ann. Geophys. 19(1), 1303-1354. 
Rème, H. et al. 1997 The CLUSTER ion spectrometry (CIS) experiment. Space Sci. Rev. 79, 303.

Retinò, A., Sundkvist, D., Vaivads, A., Mozer, F., André, M. and Owen, C. J. 2007 In situ evidence of magnetic reconnection in turbulent plasma. Nature Phys. 3, 236.

Ricci, P., Brackbill, J. U., Daughton, W. and Lapenta, G. 2004 Influence of the lower hybrid drift instability on the onset of magnetic reconnection. Phys. Plasma 11, 4489.

Robert, P., Roux, A., Harvey, C. C., Dunlop, M. W., Daly, P. W. and Glassmeier, K-H 1998 Tetrahedron geometry factors. In: Analysis Methods for Multi-Spacecraft Data (ed. G. Paschmann and P. W. Daly), Keplerlaan 1, 2200 AG Noordwijk, The Netherlands: ESA Publications Division, pp. 323-348.

Rosenbauer, H., Miggenrieder, H., Montgomery, M. D. and Schwenn, R. 1976 Preliminary results of the helios plasma experiment. In: Physics of Solar Planetary Environments (ed. D. J. Williams), Washington DC, USA: American Geophysical Union, p. 319.

Rosenbauer, H., Schwenn, R., Marsch, E., Meyer, B., H, M., Montgomery, M. D., Muhlhausser, K.-H., Pilipp, W., Voges, W. and Zink, S. M. 1977 A survey on initial results of the helios plasma experiment. J. Geophys. 42, 561-580.

Ruffolo, D., Matthaeus, W. H. and Chuychai, P. 2004 Separation of magnetic field lines in twocomponent turbulence. Astrophys. J. 614, 420-434.

Runov, A. et al. 2011a Dipolarization fronts in the magnetotail plasma sheet. Plan. Space Sci. 59, 517-525.

Runov, A., Angelopoulos, V., Sitnov, M. I., Sergeev, V. A., Bonnell, J., McFadden, J. P., Larson, D., Glassmeier, K.-H. and Auster, U. 2009 Themis observations of an earthward-propagating dipolarization front. Geophys. Res. Lett. 36, 14106.

Runov, A., Angelopoulos, V., Zhou, X.-Z., Zhang, X.-J., Li, S., Plaschke, F. and Bonnell, J. 2011b A themis multicase study of dipolarization fronts in the magnetotail plasma sheet. J. Geophys. Res. (Space Phys.) 116, 5216.

Runov, A. et al. 2003 Current sheet structure near magnetic X-line observed by Cluster. Geophys. Res. Lett. 30, 1579.

Sahraoui, F. 2008 Diagnosis of magnetic structures and intermittency in space-plasma turbulence using the technique of surrogate data. Phys. Rev. E 78, 26402.

Sahraoui, F., Belmont, G. and Goldstein, M. L. 2012 New insight into short-wavelength solar wind fluctuations from Vlasov theory. Astrophys. J. 748(2), 100.

Sahraoui, F., Belmont, G., Goldstein, M. L. and Rezeau, L. 2010a Limitations of multispacecraft data techniques in measuring wave number spectra of space plasma turbulence. J. Geophys. Res. (Space Phys.) 115, 4206.

Sahraoui, F., Belmont, G., Pinçon, J. L., Rezeau, L., Balogh, A., Robert, P. and Cornilleau-Wehrlin, N. 2004 Magnetic turbulent spectra in the magnetosheath: new insights. Ann. Geophys. 22, 2283.

Sahraoui, F., Belmont, G., Rezeau, L., Cornilleau-Wehrlin, N., Pinçon, J. L. and Balogh, A. 2006 Anisotropic turbulent spectra in the terrestrial magnetosheath as seen by the cluster spacecraft. Phys. Rev. Lett. 96, 75002.

Sahraoui, F. and Goldstein, M. 2010 Structures and intermittency in small scales solar wind turbulence. AIP Conf. Proc.: 12th Int. Solar Wind Conf., Vol. 1216, 140.

Sahraoui, F., Goldstein, M. L., Belmont, G., Canu, P. and Rezeau, L. 2010b Three-dimensional anisotropic k-spectra of turbulence at sub-proton scales in the solar wind. Phys. Rev. Lett. 105, 131 101-131 104.

Sahraoui, F., Goldstein, M. L., Robert, P. and Khotyaintsev, Y. V. 2009 Evidence of a cascade and dissipation of solar-wind turbulence at the e Electron gyroscale. Phy. Rev. Lett. 102(23), 231102.

Sahraoui, F., Huang, S. Y., Belmont, G., Goldstein, M. L., Retinò, A., Robert, P. and De Patoul, J. 2013 Scaling of the electron dissipation range of solar wind turbulence. Astrophys. J. 777(1), 15.

Sahraoui, F. et al. 2003 Ulf wave identification in the magnetosheath: the k-filtering technique applied to cluster ii data. J. Geophys. Res. (Space Phys.) 108, 1335.

Salem, C., Hubert, D., C.Lacombe, Bale, S. D., Mangeney, A., Larson, D. E. and Lin, R. P. 2003 Electron properties and Coulomb collisions in the solar wind at $1 \mathrm{AU}$ : wind observations. Astrophys. J. 585, 1147. 
Salem, C. S., Howes, G. G., D. Sundkvist, Bale, S. D., Chaston, C. C., Chen, C. H. K. and Mozer, F. S. 2012 Identification of kinetic Alfvén wave turbulence in the solar wind. Astrophys. $J$. Lett. 745, L9.

Santolík, O. 2008 New results of investigations of whistler-mode chorus emissions. Nonlinear Process. Geophys. 15, 621-630.

Santolík, O., Chum, J., Parrot, M., Gurnett, D. A., Pickett, J. S. and Cornilleau-Wehrlin, N. 2006 Propagation of whistler mode chorus to low altitudes: spacecraft observations of structured ELF hiss. J. Geophys. Res. (Space Phys.) 111(A10), 10208.

Santolik, O. and Gurnett, D. A. 2003 Transverse dimensions of chorus in the source region. Geophys. Res. Lett. 30, 1031.

Santolik, O., Gurnett, D. A., Pickett, J. S., Chum, J. and Cornilleau-Wehrlin, N. 2009 Oblique propagation of whistler-mode waves in the chorus source region. J. Geophys. Res. 114 A00F03.

Santolík, O. et al. 2010 Wave-particle interactions in the equatorial source region of whistler-mode emissions. J. Geophys. Res. (Space Phys.) 115, A00 F16.

Santolik, O., Gurnett, D. A., Pickett, J. S., Parrot, M. and Cornilleau-Wehrlin, N. 2003 Spatiotemporal structure of storm-time chorus. J. Geophys. Res. 1081278.

Santolík, O., Gurnett, D. A., Pickett, J. S., Parrot, M. and Cornilleau-Wehrlin, N. 2004 A microscopic and nanoscopic view of storm-time chorus on 31 march 2001. Geophys. Res. Lett. 31(2), L02 801.

Santolík, O., Gurnett, D. A., Pickett, J. S., Parrot, M. and Cornilleau-Wehrlin, N. 2005 Central position of the source region of storm-time chorus. Planet. Space Sci. 53, 299-305.

Santolik, O., Macusova, E., Titova, E. E., Kozelov, B. V., Gurnett, D. A., Pickett, J. S., Trakhtengerts, V. Y. and Demekhov, A. G. 2008 Frequencies of wave packets of whistler-mode chorus inside its source region: a case study. Ann. Geophys. 26, 1665-1670.

Sauer, K. and Sydora, R. D. 2010 Beam-excited whistler waves at oblique propagation with relation to STEREO radiation belt observations. $A G \mathbf{2 8}, 1317-1325$.

Schaffner, D. A., Lukin, V. S., Wan, A. and Brown, M. R. 2014 Turbulence analysis of an experimental flux-rope plasma. Plasma Phys. Contol. Fusion 56, 064003.

Schmid, D., Volwerk, M., Nakamura, R., Baumjohann, W. and Heyn, M. 2012 A statistical and event study of magnetotail dipolarization fronts. Ann. Geophys. 29, 1537-1547.

Scholer, M., Shinohara, I. and Matsukiyo, S. 2003 Quasi-perpendicular shocks: length scale of the cross-shock potential, shock reformation, and implication for shock surfing. J. Geophys. Res. (Space Phys.) 108, 1014.

Scholer, M. et al. 2005 Cluster at the bow shock: status and outlook. Space Sci. Rev. 118, 223-227.

Schriver, D. 2010 Generation of whistler mode emissions in the inner magnetosphere: an event study. J. Geophys. Res. (Space Phys.) 115, A00 F17.

Schwartz, S. J., Henley, E., Mitchell, J. and Krasnoselskikh, V. 2011 Electron temperature gradient scale at collisionless shocks. Phys. Rev. Lett. 107(2), 215002.

Sckopke, N. 1995 Ion heating at the Earth's quasi-perpendicular bow shock. Adv. Space Res. 15, 261-269.

Scudder, J. and Daughton, W. 2008 'Illuminating' electron diffusion regions of collisionless magnetic reconnection using electron agyrotropy. J. Geophys. Res. (Space Phys.) 113, 6222.

Scudder, J. D., Holdaway, R. D., Daughton, W. S., Karimabadi, H., Roytershteyn, V., Russell, C. T. and Lopez, J. Y. 2012 First resolved observations of the demagnetized electron-diffusion region of an astrophysical magnetic-reconnection site. Phys. Rev. Lett. 108, 225005.

Scudder, J. D., Mozer, F. S., Maynard, N. C. and Russell, C. T. 2002 Fingerprints of collisionless reconnection at the separator, I, Ambipolar-Hall signatures. J. Geophys. Res. 107, 1294.

Scudder, J. D., Puhl-Quinn, P. A., Mozer, F. S., Ogilvie, K. W. and Russell, C. T. 1999 Generalized walén tests through alfvén waves and rotational discontinuities using electron flow velocities. J. Geophys. Res. 104, 19817-19834.

Segalini, A., Örlü, R., Schlatter, P., Rüedi, J-D and Talamelli, A. 2011 A method to estimate turbulence intensity and transverse Taylor microscale in turbulent flows from spatially averaged hot-wire data. Exp. Fluids 51, 693-700.

Sergeev, V., Angelopoulos, V., Apatenkov, S., Bonnell, J., Ergun, R., Nakamura, R., McFadden, J., Larson, D. and Runov, A. 2009 Kinetic structure of the sharp injection/dipolarization front in the flow-braking region. Geophys. Res. Lett. 36, 21105. 
Servidio, S., Matthaeus, W. H. and Dmitruk, P. 2008 Depression of nonlinearity in decaying isotropic MHD turbulence. Phys. Rev. Lett. 100, 095005.

Servidio, S., Matthaeus, W. H., Shay, M. A., Cassak, P. A. and Dmitruk, P. 2009 Magnetic reconnection in two-dimensional magnetohydrodynamic turbulence. Phys. Rev. Lett. 102, 115003.

Servidio, S., Valentini, F., Califano, F. and Veltri, P. 2012 Local kinetic effects in two-dimensional plasma turbulence. Phys. Rev. Lett. 108, 045001.

Sitnov, M. I., Swisdak, M. and Divin, A. V. 2009 Dipolarization fronts as a signature of transient reconnection in the magnetotail. J. Geophys. Res. 114, 4202.

Sonnerup, B. Ü. Ö. and Scheible, M. 1998 Minimum and maximum variance analysis. In: Analysis Methods for Multi-Spacecraft Data (ed. G. Paschmann and P. W. Daly), Keplerlaan 1, 2200 AG Noordwijk, The Netherlands: ESA Publications Division, pp. 185-220.

Sorriso-Valvo, L., Carbone, V., Veltri, P., Consolini, G. and Bruno, R. 1999 Intermittency in the solar wind turbulence through probability distribution functions of fluctuations. Geophys. Res. Lett. 26, 1801-1804.

Stasiewicz, K. 2004 Reinterpretation of mirror modes as trains of slow magnetosonic solitons. Geophys. Res. Lett. 31, 21804.

Stasiewicz, K., Shukla, P. K., Gustafsson, G., Buchert, S., Lavraud, B., Thidé, B. and Klos, Z. 2003 Slow magnetosonic solitons detected by the Cluster spacecraft. Phys. Rev. Lett. 90(8), 085002.

Stone, E. C., Cummings, A. C., McDonald, F. B., Heikkila, B. C., Lal, N. and Webber, W. R. 2005 Voyager 1 explores the termination shock region and the heliosheath beyond. Science, Dordrecht 309, 2017-2020.

Sundberg, T., Boardsen, S. A., Slavin, J. A., Anderson, B. J., Korth, H., Zurbuchen, T. H., Raines, J. M. and Solomon, S. C. 2012 MESSENGER orbital observations of large-amplitude KelvinHelmholtz waves at Mercury's magnetopause. J. Geophys. Res. 117, 4216.

Sundkvist, D., Krasnoselskikh, V., Shukla, P. K., Vaivads, A., Andr'e, M., Buchert, S. and Rème, H. 2005 In situ multi-satellite detection of coherent vortices as a manifestation of alfvénic turbulence. Nature 436, 825-828.

Sundkvist, D., Retinó, A., Vaivads, A. and Bale, and S. D. 2007 Dissipation in turbulent plasma due to reconnection in thin current sheets. Phys. Rev. Lett. 99, 025004.

Tajima, T., Horton, W., Morrison, P. J., Schutkeker, J., Kamimura, T., Mima, K. and Abe, Y. 1991 Instabilites and vortex dynameics in shear flow of magnitized plasmas. Phys. Fluids $B$, 938-954.

Taylor, G. I. 1935 Statistical theory of turbulence. Proc. R. Soc. Lond. A 151, 421-444.

Taylor, G. I. 1938 The spectrum of turbulence. In: Proc. Royal Society of London Ser. A, pp. 476-490.

Taylor, M. G. G. T., Escoubet, C. P., Laakso, H., Masson, A. and Goldstein, M. L. 2010 The Cluster Mission: Space Plasma in Three Dimensions. Springer, Dordrecht, pp. 309-330.

Taylor, M. G. G. T. and Lavraud, B. 2008 Observation of three distinct ion populations at the Kelvin-Helmholtz-unstable magnetopause. Ann. Geophys. 26, 1559-1566.

Temerin, M., K., Cerny, W., Lotko and S., Mozer F. 1982 Observations of double layers and solitary waves in the auroral plasma. Phys. Rev. Lett. 48, 1175-1179.

Tenerani, A., Le Contel, O., Califano, F., Pegoraro, F., Robert, P., Cornilleau-Wehrlin, N. and Sauvaud, J. A. 2012 Coupling between whistler waves and ion-scale solitary waves: Cluster measurements in the magnetotail during a substorm. Phys. Rev. Lett. 109(15), 155005.

Tessein, J. A., Matthaeus, W. H., Wan, M., Osman, K. T., Ruffolo, D. and Giacalone, J. 2013 Association of suprathermal particles with coherent structures and shocks. Astrophys. J. 776, L8.

Thomsen, M. F. 1988 Multi-spacecraft observations of collisionless shocks. Adv. Space Res. 8, $157-166$.

Titov, V. S. and Hornig, G. 2000 Magnetohydrodynamic flows sustaining stationary magnetic nulls. Phys.Plasmas 7(9), 3542-3550.

Titova, E., Demekhov, A., Kozelov, B., Santolík, O., Macúšová, E., Rauch, J.-L., Trotignon, J.-G., Gurnett, D. and Pickett, J. 2012 Properties of the magnetospheric backward wave oscillator inferred from cluster measurements of vlf chorus elements. J. Geophys. Res. (Space Phys.) 117, 8210 .

Tjulin, A., Lucek, E. A. and Dandouras, I. 2008 Wave activity inside hot flow anomaly cavities. $J$. Geophys. Res. (Space Phys.) 113(A), 8113. 
Tjulin, A., Pinçon, J. L., Sahraoui, F., André, M. and Cornilleau-Wehrlin, N. 2005 The k-filtering technique applied to wave electric and magnetic field measurements from the Cluster satellites. J. Geophys. Res. 110, 11224.

Torkar, K. et al. 2001 Active spacecraft potential control for Cluster - implementation and first results. Ann. Geophys. 19(1), 1289-1302.

Trakhtengerts, V. Y. et al. 2007 Formation of VLF chorus frequency spectrum: Cluster data and comparison with the backward wave oscillator model. Geophys. Res. Lett. 34, 2104.

Treumann, R. A. and Jaroschek, C. H. 2008 Planetary bow shocks. ArXIV [astro-ph] 0808.1701, 34.

Trines, R., Bingham, R., Dunlop, M. W., Vaivads, A., Davies, J. A., Mendonça, J. T., Silva, L. O. and Shukla, P. K. 2007 Spontaneous generation of self-organized solitary wave structures at Earth's magnetopause. Phys. Rev. Lett. 99(20), 205006.

Umeda, T., Ashour-Abdalla, M., Pickett, J. S. and Goldstein, M. L. 2012 Vlasov simulation of electrostatic solitary structures in multi-component plasmas. J. Geophys. Res. (Space Phys.) $117,5223$.

Vaivads, A. A., Retinò, A. and André, M. 2006 Microphysics of magnetic reconnection. Space Sci. Rev. 122, 19.

Vasquez, B. J., Abramenko, V. I., Haggerty, D. K. and Smith, C. W. 2007 Numerous small magnetic field discontinuities of bartels rotation 2286 and the potential role of alfvénic turbulence. $J$. Geophys. Res. 112, 11102.

Veltri, P. 1999 Mhd turbulence in the solar wind: self-similarity, intermittency and coherent structures. Plasma Phys. Contol. Fusion 41, A787.

Veltri, P. and Mangeney, A. 1999 Scaling laws and intermittent structures in solar wind mhd turbulence. In: AIP Conf. Proc., Nantucket, Massachusetts (USA), p. 543.

Viberg, H., Khotyaintsev, Yu. V., Vaivads, A., André, M., Fu, H. S. and Cornilleau-Wehrlin, N. 2014 Whistler mode waves at magnetotail dipolarization fronts. J. Geophys. Res. (Space Phys.) 119, 2605-2611.

Viberg, H., Khotyaintsev, Y. V., Vaivads, A., André, M. and Pickett, J. S. 2013 Mapping HF waves in the reconnection diffusion region. Geophys. Res. Lett. 40, 1032-1037.

Viñas, A. F. and Gurgiolo, C. 2009 Spherical harmonic analysis of particle velocity distribution function: comparison of moments and anisotropies using Cluster data. J. Geophys. Res. 114, 1105.

Viñas, A. F., Gurgiolo, C., Nieves-Chinchilla, T., Gary, S. P. and Goldstein, M. L. 2010 Whistler waves driven by anisotropic 3D strahl velocity distributions in the solar wind: Cluster observations. In: AIP Proc. Solar Wind 12 Conf. (ed. M. Maksimovic, K. Issautier, N. MeyerVernet, M. Moncuquet and F. Pantellini), New York, USA: American Institute of Physics, p. 265.

Volwerk, M., Glassmeier, K.-H., Nakamura, R., Takada, T., Baumjohann, W., Klecker, B., Réme, H., Zhang, T. L., Lucek, E. and Carr, C. M. 2007 Flow burst-induced Kelvin-Helmholtz waves in the terrestrial magnetotail. Geophys. Res. Lett. 3410102.

Walker, R. J., Fukazawa, K., Ogino, T. and Morozoff, D. 2011a A simulation study of KelvinHelmholtz waves at Saturn's magnetopause. J. Geophys. Res. 116, 3203.

Walker, S. N., Balikhin, M. A., Zhang, T. L., Gedalin, M. E., Pope, S. A., Dimmock, A. P. and Fedorov, A. O. 2011b Unusual nonlinear waves in the venusian magnetosheath. J. Geophys. Res. (Space Phys.) 116, 1215.

Wan, M., Matthaeus, W. H., Karimabadi, H., Roytershteyn, V., Shay, M., Wu, P., Daughton, W., Loring, B. and Chapman, S. C. 2012 Intermittent dissipation at kinetic scales in collisionless plasma turbulence. Phys. Rev. Lett. 109, 195001.

Wang, R., Lu, Q., Du, A. and Wang, S. 2010 In Situ observations of a secondary magnetic island in an ion diffusion region and associated energetic electrons. Phys. Rev. Lett. 104, 175003.

Wang, X., Tu, C., He, J., Marschand, E. and Wang, L. 2013 On intermittent turbulence heating of the solar wind: differences between tangential and rotational discontinuities. Astrophys. $J$. Lett. 772, L14.

Wendel, D. E. and Adrian, M. L. 2013 Current structure and nonideal behavior at magnetic null points in the turbulent magnetosheath. J. Geophys. Res. (Space Phys.) 118(4), 1571-1588. 
Weygand, J. M., Matthaeus, W. H., Dasso, S. and Kivelson, M. G. 2011 Correlation and Taylor scale variability in the interplanetary magnetic field fluctuations as a function of solar wind speed. J. Geophys. Res. 116, 8102.

Weygand, J. M., Matthaeus, W. H., Dasso, S., Kivelson, M. G., Kistler, L. M. and Mouikis, C. 2009 Anisotropy of the Taylor scale and the correlation scale in plasma sheet and solar wind magnetic field fluctuations. J. Geophys. Res. 114, 7213.

Weygand, J. M., Matthaeus, W. H., Dasso, S., Kivelson, M. G. and Walker, R. J. 2007 Taylor scale and effective magnetic Reynolds number determination from plasma sheet and solar wind magnetic field fluctuations. J. Geophys. Res. 112, 10201.

Weygand, J. M., Matthaeus, W. H., El-Alaoui, M., Dasso, S. and Kivelson, M. G. 2010 Anisotropy of the Taylor scale and the correlation scale in plasma sheet magnetic field fluctuations as a function of auroral electrojet activity. J. Geophys. Res. 115, 12250.

Wilken, B. et al. 2001 First results from the RAPID imaging energetic particle spectrometer on board Cluster. Ann. Geophys. 19(1), 1355-1366.

Wilkinson, W. P. 2003 The Earth's quasi-parallel bow shock: Review of observations and perspectives for Cluster. Plan. Space Sci. 51, 629-647.

Williams, J. D., Chen, L.-J., Kurth, W. S., Gurnett, D. A. and Dougherty, M. K. 2006 Electrostatic solitary structures observed at Saturn. Geophys. Res. Lett. 33, 6103.

Wolf, R. A., Kumar, V., Toffoletto, F. R., Erickson, G. M., Savoie, A. M., Chen, C. X. and Lemon, C. L. 2006 Estimating local plasma sheet $\mathrm{PV}^{5 / 3}$ from single spacecraft observations. J. Geophys. Res. 111, 12218.

Wu, C. S. and Lee, L. C. 1979 A theory of the terrestrial kilometric radiation. Astrophys. J. 230, 621-626.

Wu, C. S. and Yoon, P. H. 1990 Kinetic hydromagnetic instabilities due to a spherical shell distribution of pickup ions. J. Geophys. Res. 95, 10273-10 278.

Wu, P., Perri, S., Osman, K., Wan, M., Matthaeus, W. H., Shay, M. A., Goldstein, M. L., Karimabadi, H., and Chapman, S. 2013 Intermittent heating in solar wind and kinetic simulations. Astrophys. J. 763, L30.

Wygant, J. R. et al. 2005 Cluster observations of an intense normal component of the electric field at a thin reconnecting current sheet in the tail and its role in the shock-like acceleration of the ion fluid into the separatrix region. J. Geophys. Res. 110, 9206.

Wyper, P. and Jain, R. 2010 Torsional magnetic reconnection at three dimensional null points: a phenomenological study. Phys. Plasmas 17, 2902.

Xiao, C. J. et al. 2007 Satellite observations of separator-line geometry of three-dimensional magnetic reconnection. Nature Phys. 3, 609-613.

Xiao, C. J. et al. 2006 In situ evidence for the structure of the magnetic null in a 3D reconnection event in the Earth's magnetotail. Nature Phys. 2, 478-483.

Yordanova, E., Vaivads, A., André, M., Buchert, S. C. and Vörös, Z. 2008 Magnetosheath plasma turbulence and its spatiotemporal evolution as observed by the cluster spacecraft. Phys. Rev. Lett. 100, 205003.

Zhou, M., Ashour-Abdalla, M., Deng, X., Schriver, D., El-Alaoui, M. and Pang, Y. 2009 THEMIS observation of multiple dipolarization fronts and associated wave characteristics in the nearearth magnetotail. Geophys. Res. Lett. 36, 20107.

Zhou, X.-Z., Angelopoulos, V., Sergeev, V. A. and Runov, A. 2010 Accelerated ions ahead of earthward propagating dipolarization fronts. J. Geophys. Res. 115, 103. 